\title{
The Universal Geometry of heterotic vacua
}

\author{
Philip Candelas, ${ }^{a}$ Xenia de la Ossa, ${ }^{a}$ Jock McOrist $^{b}$ and Roberto Sisca ${ }^{b}$ \\ ${ }^{a}$ Mathematical Institute, University of Oxford, \\ Andrew Wiles Building, Woodstock Road, \\ Radcliffe Observatory Quarter, Oxford, OX2 6GG, U.K. \\ ${ }^{b}$ Department of Mathematics, University of Surrey, \\ Guildford, GU2 7XH, U.K. \\ E-mail: candelas@maths.ox.ac.uk, delaossa@maths.ox.ac.uk, \\ j.mcorist@surrey.ac.uk, r.sisca@surrey.ac.uk
}

ABSTRACT: We consider a family of perturbative heterotic string backgrounds. These are complex threefolds $X$ with $c_{1}=0$, each with a gauge field solving the Hermitian Yang-Mill's equations and compatible $B$ and $H$ fields that satisfy the anomaly cancellation conditions. Our perspective is to consider a geometry in which these backgrounds are fibred over a parameter space. If the manifold $X$ has coordinates $x$, and parameters are denoted by $y$, then it is natural to consider coordinate transformations $x \rightarrow \tilde{x}(x, y)$ and $y \rightarrow \tilde{y}(y)$. Similarly, gauge transformations of the gauge field and $B$ field also depend on both $x$ and $y$. In the process of defining deformations of the background fields that are suitably covariant under these transformations, it turns out to be natural to extend the gauge field $A$ to a gauge field $\mathbb{A}$ on the extended $(x, y)$-space. Similarly, the $B, H$, and other fields are also extended. The total space of the fibration of the heterotic structures is the Universal Geometry of the title. The extension of gauge fields has been studied in relation to Donaldson theory and monopole moduli spaces. String vacua furnish a richer application of these ideas. One advantage of this point of view is that previously disparate results are unified into a simple tensor formulation. In a previous paper, by three of the present authors, the metric on the moduli space of heterotic theories was derived, correct through $\mathcal{O}\left(\alpha^{\prime}\right)$, and it was shown how this was related to a simple Kähler potential. With the present formalism, we are able to rederive the results of this previously long and involved calculation, in less than a page.

KEYwords: Superstring Vacua, Superstrings and Heterotic Strings

ARXiv EPrint: 1810.00879 


\section{Contents}

1 Introduction 1

1.1 Outline of the article 6

$\begin{array}{lll}1.2 & \text { Some notation and terminology } & 7\end{array}$

$\begin{array}{lll}1.3 & \text { A short summary of covariant derivation } & 8\end{array}$

2 The fibration $X \quad 8$

2.1 Defining $\boldsymbol{X} \quad 9$

2.2 Tangibility of forms 11

$\begin{array}{lll}2.3 & \text { Differential calculus on } \boldsymbol{X} & 12\end{array}$

2.4 Derivatives of tensors 14

$\begin{array}{ll}2.5 & \text { The Frölicher-Nijenhuis bracket } \\ \end{array}$

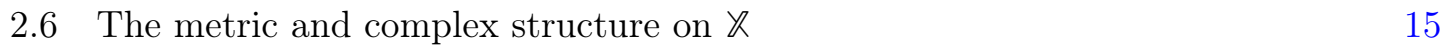

2.7 The connection $c_{a}{ }^{m}$ as the shift, and the extrinsic curvature of $X_{y} \quad 18$

3 The universal geometry $\mathcal{U} \quad 20$

$\begin{array}{lll}3.1 & \text { The extension of } A & 21\end{array}$

3.2 The extension of $B$ and $H \quad 24$

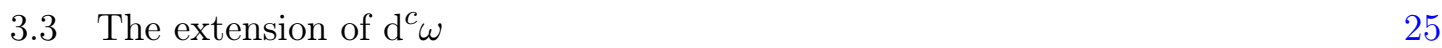

3.4 The relation $\mathbb{H}=\mathbb{d}^{c} \omega$, Bianchi identity and second order relations 26

4 Using $Х$ to deform connections on $\mathcal{T}_{X} \quad 27$

4.1 The covariant derivative of $\Theta \quad 28$

4.2 A two-parameter family of connections $\Theta^{(\epsilon, \rho)}$ on $\mathbb{X} 28$

4.3 The covariant derivative of $\Theta^{(\epsilon, \rho)} \quad 31$

4.4 The contribution of $\mathfrak{D}_{\alpha} \Theta$ to the moduli space metric 32

5 Deriving the moduli space metric from its Kähler potential 32

A Heterotic geometry $\quad 34$

A.1 Some differential geometry 34

$\begin{array}{lll}\text { A.2 Heterotic action and supersymmetry variations } & 35\end{array}$

$\begin{array}{lll}\text { A.3 The background field expansion } & 37\end{array}$

B The $\llbracket$ and $\Theta$ symbols $\quad 38$

B.1 The 『 symbols for the Levi-Civita connection 39

C The Nijenhuis tensor for $\mathbb{X} 40$

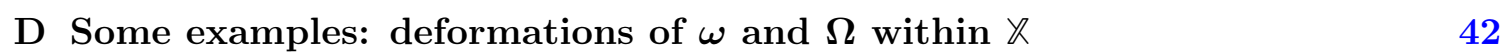

D.1 The hermitian form $\omega$

$\begin{array}{lll}\text { D.2 The holomorphic form } \Omega & 42\end{array}$ 
Nullum autem est, nisi quod animus ex se sibi invenit. There can be no good except for what the soul discovers for itself within itself.

Seneca the Younger

\section{Introduction}

Heterotic geometry is the geometry associated with the moduli space of a heterotic vacuum of string theory. The geometrical background, associated with a vacuum, is understood, at large volume, as $\mathbb{R}^{1,3} \times X$, where $X$ is a complex 3 -dimensional manifold with vanishing first Chern class. This geometry is endowed with a holomorphic vector bundle $\mathcal{E} \rightarrow X$, admitting a connection $A$ that satisfies the Hermitian Yang-Mills equations

$$
g^{\mu \bar{\nu}} F_{\mu \bar{\nu}}=0 .
$$

Also important are background values for the Kalb-Ramond field $B$ and its field strength $H$, which satisfy appropriate field equations and anomaly cancellation conditions. Heterotic geometry is the analogue of the special geometry of Type II vacua.

We term the tuple $\left([X, \omega, \Omega],[\mathcal{E}, A],\left[\mathcal{T}_{X}, \Theta\right], H\right)$ a heterotic structure and label it by Het. The data of the heterotic structure includes the connections $A$ and $\Theta$ on the bundles $\mathcal{E}$ and $\mathcal{T}_{X}$ respectively as well as the hermitian form $\omega$ and the complex structure $J$ of $X$, or equivalently the holomorphic $(3,0)$-form $\Omega$.

The metric on the moduli space of heterotic supergravity metric was computed, correct to $\mathcal{O}\left(\alpha^{\prime}\right)$ (i.e. the error is $\mathcal{O}\left(\alpha^{\prime 2}\right)$ ), in [1] by a dimensional reduction of heterotic supergravity. This metric has to be Kähler as a consequence of supersymmetry. It should not be surprising, therefore, that verifying that the moduli space is in fact Kähler requires taking into account the relations between $H$, the connection on the bundle $\mathcal{E}$, and the hermitian form $\omega$ on $X$, since these relations follow from both the anomaly cancellations condition and the requirement of supersymmetry.

Let us recall these essential conditions. The anomaly relation yields a modified Bianchi identity for $H$.

$$
\mathrm{d} H=-\frac{\alpha^{\prime}}{4}\left(\operatorname{Tr}\left(F^{2}\right)-\operatorname{Tr}\left(R^{2}\right)\right),
$$

while the supersymmetry relation takes the form ${ }^{1}$

$$
H=\mathrm{d}^{c} \omega, \quad \mathrm{d}^{c} \omega=\frac{1}{3 !} J^{m} J^{n} J^{p}(\mathrm{~d} \omega)_{m n p} .
$$

In the above equations $R$ is the curvature two-form. We denote by $x^{m}$ the real coordinates of $X$ and the holomorphic coordinates by $\left(x^{\mu}, x^{\bar{\nu}}\right)$. The vector-valued form $J^{m}=J_{n}{ }^{m} \mathrm{~d} x^{n}$ is a 1-form constructed from the complex structure. In the following we will generally omit the wedge product symbol ' $\wedge$ ' between forms, unless doing so would lead to ambiguity.

\footnotetext{
${ }^{1}$ The right hand side of this relation is often written in the form $i(\partial-\bar{\partial}) \omega$. However we prefer to write this in "real form" since this makes the calculation of the derivative of the relation with respect to the complex structure parameters more transparent.
} 
These equations already imply that the moduli space has a recondite character, since the deformations of $F, \omega$ and $H$ are intricately related. By contrast to the case of type II vacua, where the roles of the complex structure parameters and the Kähler class parameters are strictly separated, there seems to be no useful distinction, in the heterotic context, between what are conventionally labelled the complex structure moduli, hermitian moduli and bundle moduli.

The deformations of a heterotic structure, within a given topological class, correspond to the points of the moduli space $M$, which is itself a complex manifold. This has real coordinates $y^{a}$ and complex coordinates $\left(y^{\alpha}, y^{\bar{\beta}}\right)$.

We think of the heterotic structures as fibred over $M$ and denote the total space by $\mathcal{U}$. This is the universal bundle of the title. The fibration $\mathcal{U}$ is of course distinct from the holomorphic vector bundle $\mathcal{E}$, which is part of the heterotic data of each fiber of $\mathcal{U}$. Thus we have the diagram

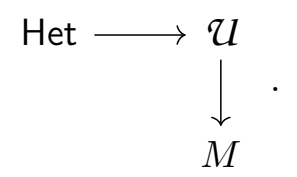

The purpose of this article is to show that considering this structure is worthwhile. Before entering into technical matters it may be helpful to indicate why this might be expected to be the case. To start, consider for example the deformation of a manifold, which is part of our data. In general relativity one often thinks of a three-geometry that evolves in time. We think of time as a parameter which governs the evolution. More generally, a manifold may depend on a number of parameters so there are a number of 'times'. A minor, but still counter-intuitive matter to a physicist, is that whereas the time axis almost always proceeds upwards in diagrams, in the fibre bundle language the parameter space is the base of the fibration and is invariably drawn horizontally while the different manifolds are the fibers and are drawn vertically. Thus time evolves sideways.

Let us take another analogy with non-relativistic electrodynamics. The fields $\mathbf{E}$ and $\mathbf{B}$ reside in $\mathbb{R}^{3}$ and evolve in time. Of course, if we pass to the four-dimensional description of relativistic dynamics, time is included as one of the coordinates. The vectors of $\mathbb{R}^{3}$, are now no longer covariant under the enlarged symmetry group of $\mathrm{SO}(1,3)$. Instead $\mathbf{E}$ and $\mathbf{B}$ come together into a two-form $F_{m n}$ that transforms covariantly under the larger group.

To see how a 'larger group' arises in the present context, consider the Yang-Mills gauge field $A$, that is present in a heterotic vacuum. It is subject to gauge transformations

$$
A \rightarrow{ }^{\Phi} A=\Phi A \Phi^{-1}-\mathrm{d} \Phi \Phi^{-1} .
$$

Since we wish to consider deformations of the gauge potential, the gauge function $\Phi$ is naturally a function of both the coordinates of $x$ of $X$ and the parameters $y$ of $M$. It is convenient to define a covariant derivative $D_{a} A$, which transforms homogeneously under gauge transformation. In order to do this in a way which takes into account the parameter dependence of the gauge transformations, one introduces also a connection $\Lambda=\Lambda_{a} \mathrm{~d} y^{a}$ on the moduli space that transforms in a manner parallel to $A$

$$
\Lambda \rightarrow{ }^{\Phi} \Lambda_{a}=\Phi \Lambda_{a} \Phi^{-1}-\partial_{a} \Phi \Phi^{-1} .
$$


The covariant derivative contains the two connections $A$ and $\Lambda$

$$
D_{a} A=\partial_{a} A-\mathrm{d}_{A} \Lambda_{a},
$$

where $\mathrm{d}_{A}$ denotes a covariant outer derivative operator

$$
\mathrm{d}_{A} \Lambda_{a}=\mathrm{d} \Lambda_{a}+\left[A, \Lambda_{a}\right] .
$$

Under a gauge transformation we see that the covariant derivative $D_{a} A$ is indeed covariant

$$
D_{a} A \rightarrow{ }^{\Phi} D_{a} A=\Phi D_{a} A \Phi^{-1} .
$$

There are certain useful identities, that can be derived in a straightforward manner that relate to this covariant derivative. Consider, for example, these two

$$
\mathrm{d}_{A}\left(D_{a} A\right)=D_{a} F, \quad \text { and } \quad\left[D_{a}, D_{b}\right] A=-\mathrm{d}_{A} \mathbb{F}_{a b},
$$

where $\mathbb{F}_{a b}=\partial_{a} \Lambda_{b}-\partial_{b} \Lambda_{a}+\left[\Lambda_{a}, \Lambda_{b}\right]$.

Let us combine the connections $A$ and $\Lambda_{a}$ into the single connection

$$
\mathbb{A}=A+\Lambda_{a} \mathrm{~d} y^{a},
$$

and form from this the associated field strength

$$
\mathbb{F}=\mathbb{d} \mathbb{A}+\mathbb{A}^{2}, \quad \text { with } \mathbb{d}=\mathrm{d}+\mathrm{d} y^{a} \partial_{a} .
$$

With this definition $\mathbb{E}$ is covariant. Moreover:

- $\mathbb{F}_{a b}$ is as defined above

- $D_{a} A=\mathbb{F}_{a m} \mathrm{~d} x^{m}$, so the covariant derivative is none other than the mixed component of the covariant field strength $\mathbb{F}$.

- Furthermore, it is easy to check that the somewhat subtle, but useful, identities (1.8) are the $(a, m, n)$ and $(a, b, m)$ components of the Bianchi identity

$$
\mathbb{d}_{\mathbb{A}} \mathbb{E}=0 .
$$

The spin connection $\Theta$ plays a role with respect to the tangent bundle $\mathcal{T}_{X}$ that is closely parallel to the role played by $A$ with respect to the vector bundle $\mathcal{E}$. Under a Lorentz transformation $\Psi$ the transformation law for $\Theta$ is

$$
\Theta \rightarrow \Psi \Theta \Psi^{-1}-\mathrm{d} \Psi \Psi^{-1} .
$$

This has field strength $R=\mathrm{d} \Theta+\Theta^{2}$, which is holomorphic and obeys the Hermitian-YangMills equation (1.1). So, for this connection, it is natural to define an extended connection and field strength analogous to those above

$$
\Theta=\Theta+\Xi_{a} \mathrm{~d} y^{a}, \quad \mathbb{R}=\mathbb{d} \Theta+\Theta^{2},
$$

where $\Xi_{a}$ is the analogue of the connection $\Lambda_{a}$. 
The point that is being made is that there is no clean separation between the coordinates $x$ of the manifold $X$ and the parameters $y$. We have seen that the gauge functions $\Phi$ and $\Psi$ are naturally functions of both $x$ and $y$. Furthermore, it is natural that the diffeomorphisms of $X$ should involve the parameters $y$. That is, we are allowed diffeomorphisms of the form

$$
x \rightarrow \widetilde{x}(x, y) ; \quad y \rightarrow \widetilde{y}(y) .
$$

This, taken together with the dependence of the gauge functions on both $x$ and $y$ constitutes the 'larger group'.

We are not the first to consider a universal bundle, in this sense. What has been said so far in this context applies to the deformation theory of gauge fields. It was originally considered by Atiyah-Singer [2], and further exploited in connection with Donaldson Theory [3-5] and its relation with five-branes [6]. Gauntlett adapted the approach of [6] to the moduli space of BPS monopoles [7]. The covariant derivatives, analogous to those constructed above, were observed to have an interpretation as the extended field strength of a universal bundle. Heterotic string theory, however, furnishes a richer, or depending on one's point of view, more complicated structure. In particular the Kalb-Ramond field $B$ is a potential of an unconventional type for the $H$ field since, technically, it is a 2-gerbe (see [8] for a broad introduction to gerbes). The field $B$ also transforms under gauge transformations and it is convenient also to try to define a covariant derivative for this field. It is not possible to define one that transforms homogeneously, so we settle for a derivative whose transformation law is as closely parallel as possible to the transformation law for $B$ itself.

As we will see later, a quantity $\mathcal{B}_{a}$ arises that is closely related to the covariant derivative, and does have the property that it transforms homogeneously. The quantity $\mathcal{B}_{a}$ is of additional interest since the combination $\mathcal{B}_{\alpha}+\mathrm{i} \mathfrak{D}_{\alpha} \omega$, where $\alpha$ is a holomorphic index and $\mathfrak{D}_{\alpha} \omega$ is a certain covariant derivative of the hermitian form $\omega$, take over the role that the derivatives of the complexified Kähler form, $\partial_{\alpha}(B+i \omega)$, play in special geometry. We will review this now, but the upshot is that extended quantities that include $\mathbb{B}$ and $\mathbb{H}$ play an important role also.

The field strength $H$ is related to $B$ by the relation

$$
H=\mathrm{d} B-\frac{\alpha^{\prime}}{4}(\operatorname{CS}[A]-\operatorname{CS}[\Theta]),
$$

where CS denotes the Chern-Simons three-form

$$
\mathrm{CS}[A]=\operatorname{Tr}\left(A \mathrm{~d} A+\frac{2}{3} A^{3}\right) .
$$

The Chern-Simons forms transform under gauge transformation and so does $B$, with the transformation law for $B$ chosen to ensure that $H$ is gauge invariant. This transformation law is

$$
{ }^{\Phi, \Psi} B=B+\frac{\alpha^{\prime}}{4}(\operatorname{Tr}(Y A-Z \Theta)+U-W),
$$

with

$$
Y=\mathrm{d} \Phi \Phi^{-1}, \quad Z=\mathrm{d} \Psi \Psi^{-1},
$$

and $U$ and $W$ are such that $\mathrm{d} U=\frac{1}{3} \operatorname{Tr}\left(Y^{3}\right)$ and $\mathrm{d} W=\frac{1}{3} \operatorname{Tr}\left(Z^{3}\right)$. 
As $H$ is gauge invariant its variation with respect to the parameters can simply be given as a partial derivative. In this way we arrive at a relation of the form

$$
\partial_{a} H=\mathrm{d} \mathcal{B}_{a}-\frac{\alpha^{\prime}}{2} \operatorname{Tr}\left(D_{a} A F\right)+\frac{\alpha^{\prime}}{2} \operatorname{Tr}\left(D_{a} \Theta R\right) .
$$

This relation identifies a gauge invariant quantity $\mathcal{B}_{a}$ that is defined up to the addition of a d-closed form.

Let us define extended forms of $B$ and $H$ that are related by

$$
\mathbb{H}=\mathbb{d} \mathbb{B}-\frac{\alpha^{\prime}}{4}(\operatorname{CS}[\mathbb{A}]-\operatorname{CS}[\Theta]), \quad \text { where } \quad \operatorname{CS}[\mathbb{A}]=\operatorname{Tr}\left(\mathbb{A} d \mathbb{A}+\frac{2}{3} \mathbb{A}^{3}\right) .
$$

It is pleasing that the important quantity $\mathcal{B}_{a}$ turns out to be a mixed component of the gauge invariant tensor $\leftrightarrow$

$$
\mathcal{B}_{a}=\frac{1}{2} \uplus_{a m n} \mathrm{~d} x^{m} \mathrm{~d} x^{n} .
$$

We take $\mathbb{H}$ to satisfy an extended supersymmetry relation and a Bianchi identity

$$
\mathbb{H}=\mathbb{d}^{c} \omega, \quad \mathbb{d} \mathbb{H}=-\frac{\alpha^{\prime}}{4}\left(\operatorname{Tr} \mathbb{F}^{2}-\operatorname{Tr} \mathbb{R}^{2}\right),
$$

whose mixed components give important relations (3.14), (3.15) and (3.17) among the heterotic moduli.

So far, we have discussed the consequence of allowing the gauge functions $\Phi$ and $\Psi$ to be functions of both $x$ and $y$. In order to discuss the extension of vectors and tensors to the bigger space we have to take into account the freedom expressed by the second of equations (1.11). We are led to introduce a covariant basis of forms and a corresponding dual basis of vectors

$$
\begin{aligned}
e^{m} & =\mathrm{d} x^{m}+c_{a}{ }^{m} \mathrm{~d} y^{a}, & e^{a} & =\mathrm{d} y^{a}, \\
e_{m} & =\partial_{m}, & e_{a} & =\partial_{a}-c_{a}{ }^{m} \partial_{m} .
\end{aligned}
$$

The quantity $c^{m}=c_{a}{ }^{m} \mathrm{~d} y^{a}$ is a connection which transforms, under $x \rightarrow \tilde{x}(x, y)$, in the form

$$
c^{\widetilde{m}}=\frac{\partial x^{\widetilde{m}}}{\partial x^{n}} c^{n}-\frac{\partial x^{\widetilde{m}}}{\partial y^{b}} \mathrm{~d} y^{b},
$$

and this ensures that the forms $e^{m}$ and vectors $e_{a}$ transform as expected

$$
e^{\widetilde{m}}=\frac{\partial x^{\widetilde{m}}}{\partial x^{n}} e^{n}, \quad e_{\widetilde{a}}=\frac{\partial y^{b}}{\partial y^{\widetilde{a}}} e_{b} .
$$

With these basis forms, we write the extended vector potential, for example, as

$$
\mathbb{A}=A_{m} e^{m}+A_{a}^{\sharp} \mathrm{d} y^{a}, \quad \text { with } \quad A_{a}^{\sharp}=\Lambda_{a}-A_{m} c_{a}{ }^{m} .
$$

We will introduce a pair of covariant derivatives $Đ$ and $\bigoplus_{a}^{\sharp}$ for the connection $c_{a}{ }^{m}$. The operator $Đ$ covariantises the de Rham operator $\mathrm{d}$ along the manifold $X$, and so within $\mathcal{X}$ defines a fibre-wise cohomology; while the operator $\mathrm{Ð}^{\sharp}$ describes how tensors on $X$ change 
under a change in parameters. In order to describe the variation of gauge dependent quantities we will also introduce a covariant derivative $\mathfrak{D}_{a}$, which is covariant with respect to both diffeomorphisms and gauge symmetries.

Inevitably, we are led to define covariant derivatives that decompose with respect to the fibre $X$ and the base $M$, and again, when we come to discuss the complex structure, with respect to the complex structures of $X$ and $M$. Thus we will have a fourfold decomposition of the derivatives, which we require to be covariant. By covariant we mean covariance with respect to the diffeomorphisms (1.11), which is more general than the situation considered in [1]. This allowed the gauge functions to be functions of both $x$ and $y$, but which only envisioned diffeomorphisms $(x, y) \rightarrow(\tilde{x}(x), \tilde{y}(y))$. The freedom to make transformations $x \rightarrow \tilde{x}(x, y)$ requires the introduction of the connection $c_{a}{ }^{m}$ and forces a redefinition of the derivatives. In an attempt to make this transition as easy as possible we have summarised these in section 1.3.

The connection $c^{m}$ can be identified with the cross term in the 'minimal' extended metric. We have two metrics that arise naturally: the metric $g_{m n}$ on the manifold $X$ and the metric $g_{a b}^{\sharp}$ on the moduli space $\mathcal{M}$. If we combine these into a 'minimal' extended metric

$$
\mathbb{d} \mathbb{s}^{2}=g_{m n} e^{m} e^{n}+g_{a b}^{\sharp} \mathrm{d} y^{a} \mathrm{~d} y^{b},
$$

and write this out in terms of the basis forms $\mathrm{d} x^{m}$ and $\mathrm{d} y^{a}$ we find the cross term

$$
\mathfrak{g}_{m a}=g_{m n} c_{a}{ }^{n} .
$$

The connection $c^{m}$ appears also in an interesting way in relation to the variation of the complex structure of $X$. The first order variation of the complex structure is recorded in a form $\Delta_{\alpha}{ }^{\mu}=\Delta_{\alpha \bar{\nu}}{ }^{\mu} \mathrm{d} x^{\bar{\nu}}$ which is defined by

$$
\Delta_{\alpha}^{\mu}=\left.\partial_{\alpha} \mathrm{d} x^{\mu}\right|^{(0,1)}
$$

It will also be shown later that $\Delta_{\alpha}{ }^{\mu}$ is also related to $c^{\mu}$ by

$$
\Delta_{\alpha} \bar{\nu}^{\mu}=-\partial_{\bar{\nu}} c_{\alpha}^{\mu} .
$$

The connection $c_{a}{ }^{m}$ also plays an important geometrical role in relation to the fibration $\mathbb{X}$, in that it determines an almost product structure $\mathbb{L}$ that provides a splitting of the tangent space of the fibration $\mathcal{T}_{\boldsymbol{X}}$ into vertical and horizontal subspaces. Being a fibration, $\mathbb{X}$ naturally encodes a vertical projection $\mathbb{X} \longrightarrow M$. A horizontal structure, equivalent to a local choice of $c_{a}{ }^{m}$, is not invariantly defined. The freedom inherent in choice of $c_{a}{ }^{m}$ corresponds precisely to the freedom to make coordinate transformations as in (1.11).

\subsection{Outline of the article}

In the body of the article we give a detailed discussion of the points outlined above.

Within the fibration $\mathcal{U}$ lies the fibration of the manifold $X$ over $\mathrm{M}$,

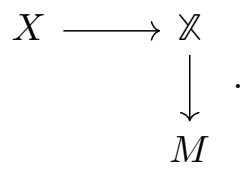


This is the natural context in which to discuss the Ehresmann connection - equivalently, the projection $\pi$ - the metric $g$ and complex structure $\mathbb{J}$ for the extended space. This is the subject of section 2. The connection $c_{a}{ }^{m}$ allows us to restrict $g$ and $\sqrt{ }$ to fibres covariantly and, when this is done, they are identified with the metric $g$ and complex structure $J$ on $X$. Furthermore, using $\pi$ we can also project $g$ and $\mathbb{I}$ to the moduli space metric $g^{\sharp}$ and complex structure $J^{\sharp}$.

In section 2, we describe the differential calculus of $\mathcal{X}$ and its relation to deformations. For example, we show that the covariant derivatives such as (1.7) are identified as Lie derivatives acting tensors on $\mathbb{X}$. This leads to an interpretation of deformations with flows on $\mathrm{X}$.

In section 3 , we start to see the profits of our labour. We introduce on $\mathbb{X}$ extensions of the connections $A$ and $\Theta$, denoted $\mathbb{A}$ and $\Theta$ respectively, which allows to discuss the extended symmetry groups mentioned above. The fields $\mathbb{A}$ and $\mathbb{\Theta}$ are holomorphic connections for the vector bundle $\mathcal{U} \rightarrow \mathfrak{X}$. Moreover, we define the extensions of $\omega$ and $H$, denoted $\omega$ and $\mathbb{H}$ respectively, and suppose a relation $\mathbb{H}=\mathbb{d}^{c} \omega$, as the extension of the supersymmetry relation (1.3).

Surprisingly, this relation together with its Bianchi identity, encapsulate in a simple pair of tensor equations, a set of long and otherwise complicated equations relating covariant derivatives which were crucial to the derivation of the Kähler moduli space metric in [1]. This is similar to how the laws of electrodynamics when viewed relativistically are unified into a simple tensor equation.

In section 4 , we illustrate a utility of $\mathbb{X}$ by showing how the curvature $\mathbb{R}$ in (1.3) can be used to compute the covariant derivative $D_{\alpha} \Theta$ in terms of the complex structure moduli $\Delta_{\alpha}{ }^{\mu}$ and hermitian moduli $\mathfrak{D}_{\alpha} \omega^{1,1}$ to zeroth order in $\alpha^{\prime}$. We then use this to compute the last term in the moduli space metric $g^{\sharp}$ derived in [1] to be

$$
\begin{aligned}
\mathrm{d} s^{\sharp 2}=2 g_{\alpha \bar{\beta}}^{\sharp} \mathrm{d} y^{\alpha} \otimes \mathrm{d} y^{\bar{\beta}} ; & \\
g_{\alpha \bar{\beta}}^{\sharp}=\frac{1}{V} \int_{X}\left\{\Delta_{\alpha}{ }^{\mu} \star \Delta_{\bar{\beta}}{ }^{\bar{\nu}} g_{\mu \bar{\nu}}\right. & +\frac{1}{4} \mathcal{Z}_{\alpha} \star \overline{\mathcal{Z}}_{\bar{\beta}}+\frac{\alpha^{\prime}}{4} \operatorname{Tr}\left(\mathfrak{D}_{\alpha} A \star \mathfrak{D}_{\bar{\beta}} A\right) \\
& \left.+\frac{\alpha^{\prime}}{2}\left(\Delta_{\alpha \overline{\mu \nu}} \Delta_{\bar{\beta} \rho \sigma}+\mathfrak{D}_{\alpha} \omega_{\rho \bar{\mu}} \mathfrak{D}_{\bar{\beta}} \omega_{\sigma \bar{\nu}}\right) R^{\bar{\mu} \rho \bar{\nu} \sigma}\right\},
\end{aligned}
$$

which generalises an expression in [9] to include all the moduli.

In section 5, we put all of this together to show how to derive the moduli space metric $g_{\alpha \bar{\beta}}^{\sharp}$ from its Kähler potential in a concise way, which simplifies much of the analysis of [1].

\subsection{Some notation and terminology}

It is useful to summarise some notation and terminology that we will introduce later.

- Tangibility $[p, q]$ means the form has $p$ legs along the moduli space $M$ and $q$ legs along the fibre $X$. Our convention is that legs along the moduli space are written first.

- The corpus of a form is the part with all legs along the fibre $X$. The animus consists of all remaining components of the form, and these are distinguished by a $\sharp$ superscript. 


\begin{tabular}{|lc|c|c|}
\hline \multicolumn{2}{|c|}{ Coordinates } & Real indices & Complex indices \\
\hline Total space $\mathbb{X}$ & $u^{P}$ & $P, Q, R, S$ & \\
\hline Base manifold $M$ & $y^{a}$ & $a, b, c, d$ & $\alpha, \beta, \gamma$ \\
\hline Fibre manifold $X$ & $x^{m}$ & $m, n, k, l$ & $\mu, \nu, \kappa, \lambda$ \\
\hline
\end{tabular}

Table 1. The coordinates and indices for the total space, fiber and base of the fibration $\mathbb{X}$.

\begin{tabular}{|c|c|c|}
\hline \multicolumn{2}{|c|}{ Relation } & Reference \\
\hline \multicolumn{2}{|c|}{$d=Đ+\bigoplus^{\sharp}$} & See section 2.3 \\
\hline & $\bigoplus^{\sharp}=\mathrm{d} y^{a} \bigoplus_{a}^{\sharp}$ & See section 2.3 \\
\hline$Đ=\check{\partial}+\bar{\varnothing}$ & $\mathrm{Ð}^{\sharp}=\widetilde{\partial}^{\sharp}+\overline{\partial^{\sharp}}$ & See section 2.5 \\
\hline \multicolumn{2}{|c|}{$\begin{array}{c}\mathfrak{D}_{a} A=e_{a}(A)-\left(Đ c_{a}{ }^{m}\right) A_{m}-\bigoplus_{A} A_{a}^{\sharp}, \\
\text { where } \bigoplus_{A} A_{a}^{\sharp}=Đ A_{a}^{\sharp}+\left[A, A_{a}^{\sharp}\right]\end{array}$} & $\begin{array}{c}\text { See section } 3.1 \\
\text { especially eq. }(3.4)\end{array}$ \\
\hline$\nabla \partial_{P}=\rrbracket^{Q}{ }_{P} \partial_{Q}$ & $\nabla e_{P}=\mathbb{\theta}^{Q} e_{Q}$ & See section 4 \\
\hline \multicolumn{2}{|c|}{$\nabla^{\mathrm{LC}}$} & Levi-Civita connection section 4.2 \\
\hline \multicolumn{2}{|c|}{$\nabla^{\mathrm{B}}$} & Bismut connection section 4.2 \\
\hline \multicolumn{2}{|c|}{$\nabla^{\mathrm{H}}$} & Hull connection section 4.2 \\
\hline \multicolumn{2}{|c|}{$\mathfrak{D}_{\alpha} \Theta^{\mathrm{H}}=\nabla\left(\Delta_{\alpha}\right)+i \nabla \mathfrak{D}_{\alpha} \omega^{(1,1)}$} & Variation of $\Theta$. See section 4.3 \\
\hline
\end{tabular}

Table 2. A table of derivatives used in the paper.

\subsection{A short summary of covariant derivation}

We have need of derivatives that are covariant under the coordinate transformations (1.11), this requires a refinement of the derivatives defined in [1] for which covariance was required only under the simpler transformations $(y, x) \rightarrow(\tilde{y}(y), \tilde{x}(x))$. We are led to construct outer derivatives that descend from $\mathbb{d}$ and covariant derivatives $\bigoplus_{a}^{\sharp}$ and $\mathfrak{D}_{a}$. For complex manifolds $X$ and $M$ the operators $Đ$ and $\bigoplus^{\sharp}$ split further into $\partial+\bar{\partial}$ and $\varnothing^{\sharp}+\overline{\partial^{\sharp}}$, which are the analogues of the familiar split $\mathrm{d}=\partial+\bar{\partial}$.

Furthermore, we overload the derivative symbol so that $\mathrm{Ð}_{a}^{\sharp}$, say, should also be covariant with respect to gauge transformations. When we take into account the complex structure of $X$ and $M$, the $\bigoplus_{a}^{\sharp}$ decomposes further into $\mathfrak{D}_{\alpha}$ and $\mathfrak{D}_{\bar{\beta}}$, which are suitable generalisations of the holotypical derivatives of [1]. From section 3.3 we write $\mathfrak{D}$ in place of $\bigoplus^{\sharp}$ even when acting on 'gauge neutral' objects since no ambiguity arises, and this gives cleaner expressions. For example, we understand that $\mathfrak{D}_{\alpha} \omega=\bigoplus_{\alpha}^{\sharp} \omega$.

\section{The fibration $\backslash$}

Our goal is to realise the embedding of heterotic structures Het, corresponding to the tuple $\left([X, \omega, \Omega],[\mathcal{E}, A],\left[\mathcal{T}_{X}, \Theta\right], H\right)$, and their parameter space $M$ inside a single object, which is a fibration $\mathcal{U}$. In this paper we will not discuss singularities of the heterotic structure, leaving these global issues for future work. Differential calculus on $\mathcal{U}$ will then account for 
the variations of a heterotic structure as one moves across $M$. We start by first considering variations of the manifold $X$ over $M$. We will include the vector bundle $\mathcal{E}$, three-form $H$ and the constraints on these objects deriving from the anomaly and supersymmetry later. To describe these variations we consider a fibration $\mathbb{X}$ which represents the family of complex manifolds $X$ with $c_{1}=0$ and conformally balanced metric over the moduli space.

\section{$2.1 \quad$ Defining $\backslash$}

The fibration $\mathbb{X}$ is pictured as

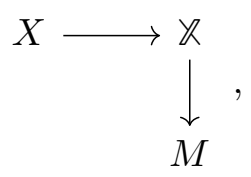

it has the following properties:

1. The fibres of $\mathbb{X}$ corresponding to the manifolds $X$, and all the fibres are diffeomorphic.

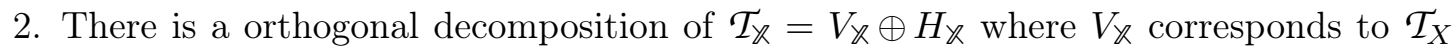
and $H_{\backslash}$ is an orthogonal complement; sections of $H_{\boldsymbol{X}}$ will correspond to deformations in an appropriate way.

We introduce local coordinates $u=(y, x)$ for $\mathfrak{X}$, with notation as displayed in the table 1. This is such that, for a fixed $y, V_{\boldsymbol{K}}=\operatorname{span}\left\{\partial_{m}\right\}$ is the subbundle of $\mathcal{T}_{\boldsymbol{K}}$ identified

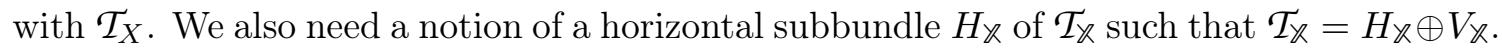
This is facilitated by introducing a projection operator $\pi: \mathcal{T}_{\text {久 }} \rightarrow \mathcal{T}_{\mathbf{X}}$ :

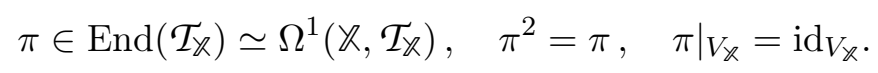

This operator $\pi$ is referred to as an Ehresmann connection in the literature. The most general expression for projection operator $\pi$ satisfying the conditions above is

$$
\pi=\mathrm{d} x^{m} \otimes \partial_{m}+c_{a}{ }^{m} \mathrm{~d} y^{a} \otimes \partial_{m},
$$

for suitable quantities $c_{a}{ }^{m}$. This connection provides the desired orthogonal decomposition

$$
\mathcal{T}_{\text {久 }}=H_{\text {久 }} \oplus V_{\text {久 }}=\operatorname{ker} \pi \oplus \operatorname{im} \pi,
$$

where the horizontal and vertical subbundles, together with their duals, are spanned by the following basis vectors and forms

$$
\begin{array}{ll}
H_{\mathfrak{X}}=\operatorname{span}\left\{e_{a}=\partial_{a}-c_{a}{ }^{m} \partial_{m}\right\}, & V_{\mathfrak{X}}=\operatorname{span}\left\{e_{m}=\partial_{m}\right\}, \\
H_{\mathfrak{X}}^{*}=\operatorname{span}\left\{e^{a}=\mathrm{d} y^{a}\right\}, & V_{X^{*}}=\operatorname{span}\left\{e^{m}=\mathrm{d} x^{m}+c_{a}{ }^{m} \mathrm{~d} y^{a}\right\} .
\end{array}
$$

We will refer to this as the "e-basis" for the tangent and cotangent space of $\mathbb{X}$. This is also referred to as a non-holonomic basis in the literature. This decomposition into horizontal and vertical subspace is referred to as an almost product structure.

The projection operator $\pi$ in the e-basis takes the form $\pi=e^{m} \otimes \partial_{m}$. We can define a related tensor $\mathbb{L}=1-2 \pi$ which satisfies the property that $\mathbb{L}^{2}=1$. This tensor defines an 
almost product structure, and is the natural analogue of an almost complex structure. We refer the reader to [10] for a comprehensive introduction. In the $e$-basis $\mathbb{L}$ is diagonal with eigenvalues +1 for the horizontal subbundle $H_{\text {久 }}$ and -1 for the vertical subbundle $V_{\text {久 }}$.

The horizontal subbundle $H_{\text {久 }}$ is integrable in the sense of Frobenius's theorem if the Lie bracket of two horizontal vectors is also a horizontal vector. That is,

$$
\left[h_{1}, h_{2}\right] \in H_{\text {久 }} \quad \text { for } \quad h_{1}, h_{2} \in H_{\text {久 }} .
$$

A short computation shows this is satisfied if and only if the Lie bracket of the basis vectors $e_{a}$ vanishes

$$
\left[e_{a}, e_{b}\right]=0 .
$$

As we will see, we can view the symbols $c_{a}{ }^{m}$ as a connection on $M$ with curvature $S$ which is a vector valued two-form with components:

$$
S_{a b}{ }^{m}=\left[e_{a}, e_{b}\right]^{m}=c_{a}{ }^{m}, b-c_{b}{ }^{m}, a+c_{a}{ }^{n} c_{b}{ }^{m}, n-c_{b}{ }^{n} c_{a}{ }^{m}, n .
$$

The tensor $\mathbb{L}$ has a Nijenhuis tensor defined as

$$
N_{\mathbb{L}}=\left(\mathbb{L}^{P} \partial_{P} \mathbb{L}^{Q}-\mathbb{L}_{P}{ }^{Q} \mathbb{\mathbb { L }}{ }^{P}\right) \partial_{Q},
$$

where $u^{P}=\left(y^{a}, x^{m}\right)$ denotes a point in $\mathbb{X}$ and we have written

$$
\mathbb{L}^{P}=\mathbb{L}_{S}^{P} \mathrm{~d} u^{S} .
$$

Another short computation shows that

$$
N_{\mathbb{L}}=\frac{1}{4} S_{a b} \mathrm{~d} y^{a} \mathrm{~d} y^{b} .
$$

Hence, vanishing of the Nijenhuis tensor $N_{\mathbb{L}}$ is equivalent to vanishing $S_{a b}$ and this is in turn equivalent to the horizontal subbundle being integrable in the sense of (2.6). Geometrically, it means that $\mathbb{X}$ is foliated with $H_{\mathbf{X}}$ being the tangent bundle to codimension six leaves in $\mathbb{X}$. That is, the tangent space of $M$ is identified with $H_{\chi}$ and $M$ is a submanifold of $\mathbb{X}$. We will see later that the vanishing of the Lie bracket above is equivalent to demanding deformations of heterotic structures commute. Frobenius's theorem implies that given $N_{\mathbb{L}}=0$ we can find a set of coordinates in which $c_{a}=0$. Diffeomorphisms that preserve this are of the form $\widetilde{x} \rightarrow \widetilde{x}^{\prime}(\widetilde{x})$ and $\widetilde{y} \rightarrow \widetilde{y}^{\prime}(\widetilde{y})$.

In the following, in the interest of generality - possibly with a view to describing non-commuting deformation theory — we will not assume $S$ vanishes except in the final two sections when we apply it to heterotic geometry.

When no additional structures are present, the automorphism group of $\mathbb{X}$ consists of bundle diffeomorphisms, that preserve the bundle structure. A key property of such a diffeomorphism $\tau$ is that the following diagram should commute:

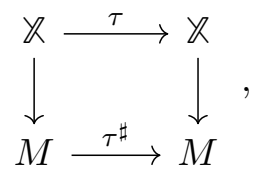


where $\tau^{\sharp}: M \rightarrow M$ is the naturally induced map. Under such a diffeomorphism the fibre $X_{y}$ is mapped onto the fibre $X_{\tau^{\sharp}(y)}$ and there is no intersection of fibres. In other words, at least locally, there is a unique manifold $X_{y}$ corresponding to each point in the base $M$. In our coordinate system $\left(y^{a}, x^{m}\right)$ a bundle diffeomorphism acts as

$$
\left(y^{a}, x^{m}\right) \stackrel{\tau}{\longrightarrow}\left(\tau^{a}(y), \tau^{m}(y, x)\right) .
$$

We can now see that under $\tau$, the symbols $c_{a}{ }^{m}$ transform as a connection

$$
{ }^{\tau} c_{a}{ }^{m}=\left(\frac{\partial \tau^{m}}{\partial x^{n}} c_{b}{ }^{n}-\frac{\partial \tau^{m}}{\partial y^{b}}\right) \frac{\partial y^{b}}{\partial \tau^{a}} .
$$

This is just the transformation law given in (1.16). With this transformation law the basis elements $e_{a}, e_{m}$ in (2.5) are covariant and span invariant subspaces.

Consider a $\tau$ that is the identity map when restricted to the base manifold $M$ :

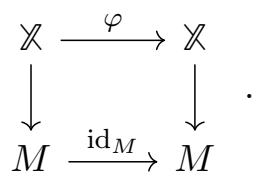

This acts as

$$
\left(y^{a}, x^{m}\right) \stackrel{\varphi}{\longrightarrow}\left(y^{a}, \varphi^{m}(y, x)\right) .
$$

These transformations correspond to the action of the structure group on $\mathcal{X}$. Since $X$ is a manifold the structure group is $\operatorname{Diff}(X)$, which is infinite dimensional and non compact, so the bundle $\mathbb{X}$ is quite different from a principal bundle or a vector bundle. For example, the principal bundle associated to $\mathbb{X}$ is not a manifold.

\subsection{Tangibility of forms}

An $n$-form $\eta$ on $X$ extends to a form $\eta$ on $\backslash$ X. In a coordinate basis this takes the form

$$
\text { ๆ }=\frac{1}{n !} \eta_{a_{1} a_{2} \cdots a_{n}} \mathrm{~d} y^{a_{1} \cdots a_{n}}+\frac{1}{(n-1) !} \eta_{a_{1} \cdots a_{n-1} m} \mathrm{~d} y^{a_{1} \cdots a_{n-1}} \mathrm{~d} x^{m}+\cdots+\frac{1}{n !} \eta_{m_{1} \cdots m_{n}} \mathrm{~d} x^{m_{1} \cdots m_{n}},
$$

where $\mathrm{d} y^{a_{1} a_{2} \cdots a_{n}}=\mathrm{d} y^{a_{1}} \mathrm{~d} y^{a_{2}} \cdots \mathrm{d} y^{a_{n}}$ and $\mathrm{d} x^{m_{1} m_{2} \cdots m_{n}}=\mathrm{d} x^{m_{1}} \mathrm{~d} x^{m_{2}} \cdots \mathrm{d} x^{m_{n}}$. This expression does not manifestly respect the symmetries of $\mathbb{X}$ under bundle diffeomorphisms (2.10), and so is not convenient. Instead, we will always decompose forms in the $e$-basis

$$
\eta=\frac{1}{n !} \eta^{\sharp} a_{1} a_{2} \cdots a_{n} \mathrm{~d} y^{a_{1} \cdots a_{n}}+\frac{1}{(n-1) !} \eta^{\sharp} a_{1} \cdots a_{n-1} m \mathrm{~d} y^{a_{1} \cdots a_{n-1}} e^{m}+\cdots+\frac{1}{n !} \eta_{m_{1} \cdots m_{n}} e^{m_{1} \cdots n_{n}},
$$

where we adopt the convention of denoting components of forms in this basis by a $\sharp$, and note that $\eta^{\sharp} m_{1} \cdots m_{n}=\eta_{m_{1} \cdots m_{n}}$. We also order forms such that the $\mathrm{d} y$ 's are written first.

We divide this decomposition into its corpus and animus: the corpus being the unextended part, $\eta_{m_{1} \cdots m_{n}} e^{m_{1}} \cdots e^{m_{n}}$, and the animus being the remainder, including the mixed $\mathrm{d} y^{a} e^{m}$ terms. The components of the corpus are always identified with the components of the original unextended form on the manifold $X$. Since we will sometimes want to break 
up the forms according to their ranks as form on $X$ and $M$ we will make reference to the tangibility of the parts. We define the tangibility of each term in (2.13) as the pair $[p, q]$ in which $q$ denotes the number of corporal indices.

$$
\eta=\sum_{p+q=n} \prod^{[p, q]} .
$$

Thus the corpus of $\emptyset$ has tangibility $[0, n]$ while the animus is the remainder, including the mixed terms: the key virtue of this decomposition is that different tangibilities do not mix under bundle diffeomorphisms. We use square brackets to distinguish tangibility from holomorphic type.

\subsection{Differential calculus on $\mathbb{X}$}

We now introduce a differential calculus on $\mathfrak{X}$ that will eventually describe how heterotic structures vary over the moduli space $M$.

The de Rham operator on $\mathbb{X}$, which defines the exterior derivative, is denoted by

$$
\mathbb{d}: \Omega^{n}(\mathbb{X}) \rightarrow \Omega^{n+1}(\mathbb{X}) .
$$

When acting on the basis of forms $\left\{\mathrm{d} y^{a}, e^{m}\right\}$ it gives $^{2}$

$$
\mathbb{d}\left(\mathrm{d} y^{a}\right)=0, \quad \mathbb{d} e^{m}=-\partial_{n} c_{a}^{m} \mathrm{~d} y^{a} e^{n}-\frac{1}{2} S_{a b}{ }^{m} \mathrm{~d} y^{a} \mathrm{~d} y^{b}
$$

where the second relation has parts of tangibility $[1,1]$ and $[2,0]$. This implies that $\mathbb{d}$ acting on a $[0, q]$ form $\eta$ is

$$
\mathbb{d} \eta=(\mathbb{d} \eta)^{[0, q+1]}+(\mathbb{d} \eta)^{[1, q]}+(\mathbb{d} \eta)^{[2, q-1]},
$$

where the different tangibilities are given by

$$
\begin{aligned}
(\mathbb{d} \eta)^{[0, q+1]} & =\frac{1}{q !}\left(\partial_{n} \eta_{m_{1} \cdots m_{q}}\right) e^{n} e^{m_{1} \cdots m_{q}} \\
(\mathbb{d} \eta)^{[1, q]} & =\frac{1}{q !}\left(e_{a}\left(\eta_{m_{1} \cdots m_{q}}\right)-c_{a}{ }^{n}{ }_{, m_{1}} \eta_{n m_{2} \cdots m_{q}}-\cdots-c_{a}{ }^{n}{ }_{, m_{q}} \eta_{m_{1} \cdots m_{q-1} n}\right) \mathrm{d} y^{a} e^{m_{1} \cdots m_{q}} \\
(\mathbb{d} \eta)^{[2, q-1]} & =-\frac{1}{2(q-1) !} S_{a b}{ }^{n} \eta_{n m_{1} \cdots m_{q-1}} \mathrm{~d} y^{a b} e^{m_{1} \cdots m_{q-1}}
\end{aligned}
$$

where in the second line we have denoted the non-holonomic derivative as $e_{a}\left(\eta_{m_{1} \cdots m_{q}}\right)=\left(\partial_{a}-\right.$ $\left.c_{a}{ }^{n} \partial_{n}\right) \eta_{m_{1} \cdots m_{q}}$. This is also referred to as a pfaffian derivative in the mathematics literature. We see that, in general, a tangibility $[p, q]$ form, with $q \geq 1$, is mapped to a form with three

\footnotetext{
${ }^{2} \mathrm{We}$ are redefining the operator $\mathrm{d}$, so the differentials, hitherto denoted by $\mathrm{d} y^{a}$, should now be written $\mathbb{d} y^{a}$. Since $\mathrm{d}$ now denotes the $x$-part of $\mathbb{d}$, we have $\mathrm{d}\left(y^{a}\right)=0$. We will however abuse notation by continuing to write $\mathrm{d} y^{a}$, when we mean $d y^{a}$.
} 
different tangibilities divided up into branches as shown

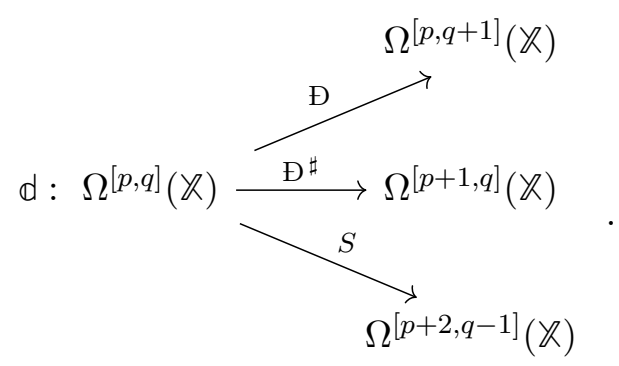

The presence of three possible branches is analogous to a non-integrable complex structure in which the exterior derivative of a holomorphic type $(p, q)$ form has non-vanishing $(p+2, q-1)$ and $(p-1, q+2)$ projections which depend on the Nijenhuis tensor. Note that, we get a reduction to two tangibilities as the horizontal subbundle is integrable but we have illustrated the presence of $S$ for generality.

The first two branches are defined by two covariant derivatives $Đ$ and $\mathrm{Ð}^{\sharp}$

$$
\begin{aligned}
& \text { Đ : } \Omega^{[p, q]}(\mathbb{X}) \rightarrow \Omega^{[p, q+1]}(\mathbb{X}), \\
& \bigoplus^{\sharp}: \Omega^{[p, q]}(\mathbb{X}) \rightarrow \Omega^{[p+1, q]}(\mathbb{X}) .
\end{aligned}
$$

They are defined by specifying their action on a zero-form $f$, a function, and on the basis $\left\{\mathrm{d} y^{a}, e^{m}\right\}$ of one-forms: ${ }^{3}$

$$
\begin{array}{llrl}
Ð f=\left(\partial_{m} f\right) e^{m}, & Đ e^{m}=0, & Đ\left(\mathrm{~d} y^{a}\right)=0, \\
\bigoplus^{\sharp} f=e_{a}(f) \mathrm{d} y^{a}, & \bigoplus^{\sharp} e^{m}=-c_{a}{ }^{m},{ }_{n} \mathrm{~d} y^{a} e^{n}, & \bigoplus^{\sharp}\left(\mathrm{d} y^{a}\right)=0 .
\end{array}
$$

It is straightforward to check that

$$
\bigoplus^{2}=0 \quad \text { and } \quad\left\{Đ, \boxplus^{\sharp}\right\}=0 .
$$

It is straightforward to check that the second line of (2.14) is consistent with applying the rules (2.16) to compute $\mathrm{Ð}^{\sharp} \eta$ :

$$
\bigoplus^{\sharp} \eta=\mathrm{d} y^{a} \bigoplus_{a}^{\sharp} \eta,
$$

where we have defined a shorthand

$$
\begin{aligned}
\bigoplus_{a}^{\sharp} \eta & =\frac{1}{q !}\left(e_{a}\left(\eta_{m_{1} \cdots m_{q}}\right)-c_{a}{ }^{n}, m_{1} \eta_{n m_{2} \cdots m_{q}}-c_{a}{ }^{n}, m_{2} \eta_{m_{1} n \cdots m_{q}}-\cdots\right) e^{m_{1}} \cdots e^{m_{q}} \\
& =e_{a}(\eta)-\left(Đ c_{a}{ }^{m}\right) \eta_{m} .
\end{aligned}
$$

In passing to the second line we have used $e_{a} \cdot e^{m}=0$ and

$$
\eta_{m}=\frac{1}{(q-1) !} \eta_{m n_{1} \ldots n_{q-1}} e^{n_{1}} \cdots e^{n_{q-1}}
$$

The derivative $\mathrm{Ð}_{a}^{\sharp} \eta$ describes a variation of $\eta$ in the direction of a vector $e_{a}$, along the moduli space. Notice that this is identified with the Lie derivative $\mathcal{L}_{e_{a}} \eta$.

\footnotetext{
${ }^{3}$ Note that it is not the case that $Đ=e^{m} \partial_{m}$ and $\bigoplus^{\sharp}=\mathrm{d} y^{a} \partial_{a}$.
} 
Both the $Đ$-operator and $\mathrm{Ð}^{\sharp}$-operator are invariant under diffeomorphisms. This is most easily seen by projecting onto tangibility, we have for example $\bigoplus^{\sharp} \eta=(\mathbb{d} \eta)^{[1, q]}$.

In [1] we implicitly assumed that variations could be written by partial derivatives, for example that the first order deformation of $\omega$ is given by $\delta \omega=\mathrm{d} y_{a} \partial_{a} \omega$. Now we see that we should write

$$
\delta \omega=\mathrm{d} y^{a} \bigoplus_{a}^{\sharp} \omega .
$$

We also have need for second order variations of fields. In this case, the curvature $S$ introduces a commutator:

$$
\left[\bigoplus_{a}^{\sharp}, \bigoplus_{b}^{\sharp}\right] \omega=S_{a b}^{m}(Đ \omega)_{m}+Đ\left(S_{a b}^{m} \omega_{m}\right) .
$$

So we see that demanding that $\pi$ be integrable, so that $S=0$, is equivalent to demanding that all variations commute and that $\left(\bigoplus^{\sharp}\right)^{2}=0$. The bundle then becomes locally flat. As previously mentioned we will keep $S$ around in the interest of generality, and set it to zero at the end.

To summarise: in general, $\mathbb{d} \eta$ decomposes into three terms,

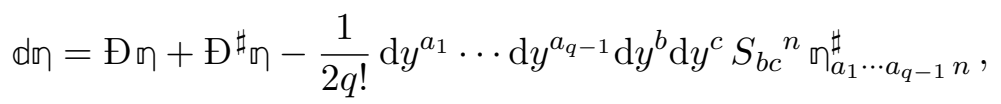

with the parts given by (2.18) as above. We have the identities

$$
\mathbb{d}^{2}=0, \oplus^{2}=0 \quad \text { and } \quad\left\{Đ, \oplus^{\sharp}\right\}=0 .
$$

If, in addition, the curvature $S$ vanishes, then we have also

$$
\mathbb{d}=Đ+\bigoplus^{\sharp} \text { and }\left(\bigoplus^{\sharp}\right)^{2}=0 .
$$

\subsection{Derivatives of tensors}

Consider a tensor $\xi$ whose indices are purely vertical:

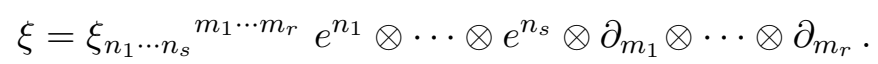

The deformation of this tensor in the direction of a horizontal vector $Y^{\sharp}=Y^{a}(y) e_{a}$ is given by a Lie derivative

$$
\mathcal{L}_{Y^{\sharp}} \xi=Y^{a} \mathcal{L}_{e_{a}} \xi .
$$

We take horizontal vector fields $Y^{\sharp}$ to depend only on the parameters. This Lie derivative then acts as a directional derivative along the moduli space. ${ }^{4}$

It is useful to describe the action of the Lie derivative in this way in components. Its action in the direction $e_{a}$ is determined by

$$
\mathcal{L}_{e_{a}} f=e_{a}(f), \quad \mathcal{L}_{e_{a}}\left(\partial_{m}\right)=c_{a}{ }^{n}, m \partial_{n}, \quad \mathcal{L}_{e_{a}}\left(e^{m}\right)=-c_{a}{ }^{m}{ }_{, n} e^{n}-S_{a b}{ }^{m} \mathrm{~d} y^{b} .
$$

\footnotetext{
${ }^{4}$ This does not hold generally for the Lie derivative. For instance the $\mathcal{L}_{f Y}(X)=f \mathcal{L}_{Y}(X)-Y(f) X$, where $f$ is a function and $X, Y$ two arbitrary vectors.
} 
Therefore, the Lie derivative of $\xi$ along the horizontal vector $e_{a}$ has vertical components given by

$$
\begin{gathered}
\mathcal{L}_{e_{a}} \xi_{n_{1} \cdots n_{s}}{ }^{m_{1} \cdots m_{r}}=e_{a}\left(\xi_{n_{1} \cdots n_{s}}{ }^{m_{1} \cdots m_{r}}\right)+c_{a}{ }^{m_{1}}{ }_{, k} \xi_{n_{1} \cdots n_{s}}{ }^{k m_{2} \cdots m_{r}}+\cdots+c_{a}{ }^{m_{r}}, k \\
\xi_{n_{1} \cdots n_{s}}{ }^{m_{1} \cdots m_{r-1} k} \\
-c_{a}{ }^{k}, n_{1} \xi_{k n_{2} \cdots n_{s}}{ }^{m_{1} \cdots m_{r}}-\cdots-c_{a}{ }^{k}{ }_{, n_{s}} \xi_{n_{1} \cdots n_{s-1} k}{ }^{m_{1} \cdots m_{r}} .
\end{gathered}
$$

When applied to a differential form $\eta$ reduces to the form given by (2.17).

We can relate this to the derivative $\mathrm{\Xi}^{\sharp}$. The covariant derivative with respect parameters is

$$
\bigoplus_{a}^{\sharp} f=e_{a}(f), \quad \bigoplus_{a}^{\sharp} \partial_{m}=c_{a}{ }^{n}, m \partial_{n}, \quad \bigoplus_{a}^{\sharp} e^{m}=-c_{a}{ }^{m}{ }_{, n} e^{n},
$$

and this is the projection of (2.19) onto the fibers. This amounts to dropping the term proportional to $S$ so, when $\pi$ is integrable, derivatives of tensors are described exactly by $\bigoplus^{\sharp}$.

\subsection{The Frölicher-Nijenhuis bracket}

The Frölicher-Nijenhuis (FN) bracket extends the Lie bracket on vector fields to vector field valued forms:

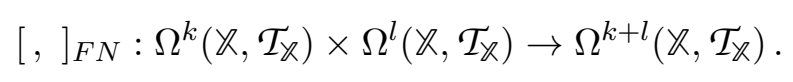

We are interested in the case $k=l=1$. Given $\mathbb{K}, \mathbb{L} \in \Omega^{1}\left(\mathcal{X}, \mathcal{T}_{\mathcal{X}}\right)$ and vectors $\vee, \mathfrak{w}$ we have

$$
[\mathbb{L}, \mathbb{K}]_{F N}(\mathbb{v}, \mathfrak{W})=[\mathbb{L} v, \mathbb{K} \mathfrak{w}]+[\mathbb{K} v, \mathbb{L} \mathfrak{w}]+(\mathbb{K} \mathbb{L}+\mathbb{L} \mathbb{K})[\mathbb{v}, \mathfrak{W}]-\mathbb{K}([\mathbb{L} v, \mathfrak{w}]+[\mathbb{v}, \mathbb{L} \mathfrak{w}])-\mathbb{L}([\mathbb{K} \mathbb{v}, \mathfrak{w}]+[\mathbb{v}, \mathbb{K} \mathbb{w}]),
$$

where the brackets on the right-hand side are the usual Lie brackets. The Nijenhuis tensors of $\mathbb{L}$ and $\mathbb{K}$ are expressible in terms of the Frölicher-Nijenhuis bracket:

$$
N_{\mathbb{L}}=\frac{1}{2}[\mathbb{L}, \mathbb{L}]_{F N}, \quad N_{\mathbb{K}}=\frac{1}{2}[\mathbb{K}, \mathbb{K}]_{F N} .
$$

\subsection{The metric and complex structure on $\backslash$}

We now put a metric and complex structure on $\mathbb{X}$ in a way that appropriately reflects the fact we are studying the moduli of heterotic structures. We start with a metric on $\mathbb{X}$. For the vertical fibers $X_{y}$ we have a family of metrics $g_{m n}(y, x) \mathrm{d} x^{m} \otimes \mathrm{d} x^{n}$, and for the moduli space $M$ we have a natural metric $g_{a b}^{\sharp}(y) \mathrm{d} y^{a} \otimes \mathrm{d} y^{b}$, which, correct through $\mathcal{O}\left(\alpha^{\prime}\right)$, is given by (1.18). The most straightforward way to combine these parts is to require the metric to be compatible with the projection operator $\pi$ which amounts to the metric being block diagonal in the $e$-basis.

$$
\mathrm{d} s^{2}=g_{m n}(y, x) e^{m} \otimes e^{n}+g_{a b}^{\sharp}(y) \mathrm{d} y^{a} \otimes \mathrm{d} y^{b} .
$$

With respect to this metric, the spaces $V_{\swarrow}$ and $H_{\swarrow}$ are orthogonal.

In more erudite language: we note that since $V_{\mathfrak{X}} \cong \mathcal{T}_{X}$ we should identify the inner product on $V_{\boldsymbol{\chi}}$ with the metric on $X$. For the horizontal part, first note that $\left.\mathcal{T}_{M}\right|_{y}$ and $\left.H_{\bigvee}\right|_{(y, x)}$ are isomorphic as vector spaces. Demanding compatibility between the two projections $\mathcal{T}_{\mathfrak{K}} \rightarrow \mathcal{T}_{M}$ and $\mathcal{T}_{\mathfrak{K}} \rightarrow H_{\mathfrak{X}}$, so the horizontal metric needs to coincide with the moduli metric. This leads to the form given. 
The given form of the metric is not unique, since the components of the connection $c_{a}{ }^{m}$ are not specified. In a coordinate basis we have

$$
\mathbb{I}_{m a}=g_{m n} c_{a}{ }^{n}
$$

So the off-diagonal blocks of the metric have been left, so far, unspecified.

The fibers of $\mathcal{X}$ and also the base $M$ are complex manifolds; we ask if $\mathcal{X}$ is also. Denoting the complex structures of $X$ and $M$ by $J$ and $J^{\sharp}$, we can combine them into an almost complex structure $\mathbb{J}$ for $\mathcal{X}$ in a manner analogous to the construction of the metric

$$
\mathbb{J}=J^{\sharp}{ }_{b}^{a} \mathrm{~d} y^{b} \otimes e_{a}+J_{n}{ }^{m} e^{n} \otimes \partial_{m} .
$$

This is tantamount to demanding that $\sqrt{ }$ and $\pi$ commute as endomorphisms, but not as differential structures. Demanding they commute as differential structures means, essentially, that $[\mathbb{J}, \mathbb{L}]_{F N}=0$, which is too strong for our situation. It is immediate that $\mathbb{J}^{2}=-\mathbb{1}$, without further conditions on the connection $c_{a}{ }^{m}$.

We examine next the Nijenhuis tensor of $\mathbb{J}$. In appendix $\mathrm{C}$ we show that this has the form

$N_{\unlhd}=-2\left(2\left[P_{a}^{\sharp}, P_{m}\right]^{q} e^{a} e^{m} Q_{q}+2\left[Q_{a}^{\sharp}, Q_{m}\right]^{q} e^{a} e^{m} P_{q}+\left[P_{c}^{\sharp}, P_{d}^{\sharp}\right]^{q} e^{c} e^{d} Q_{q}+\left[Q_{c}^{\sharp}, Q_{d}^{\sharp}\right]^{q} e^{c} e^{d} P_{q}\right)$,

where $P_{m}=P_{m}{ }^{n} \partial_{n}$ is a vector constructed out of the projector $P_{m}^{n}$, and $P_{a}^{\sharp}$ is constructed analogously. The term $\left[P_{c}^{\sharp}, P_{d}^{\sharp}\right]$ is the $(2,0)$-component of the curvature $S$. If the manifold is complex, which is the case $N_{\S}=0$, then we can write these terms in complex coordinates $\left(y^{\alpha}, y^{\bar{\beta}}, x^{\mu}, x^{\bar{\nu}}\right)$. The vanishing of the right hand side requires

$$
\left[e_{\alpha}, \partial_{\mu}\right]^{\bar{\nu}}=0, \quad \text { and } \quad\left[e_{\alpha}, e_{\beta}\right]^{\bar{\nu}}=0 .
$$

These equations are then constraints on the connection $c_{a}{ }^{m}$ :

$$
\begin{aligned}
& 0=\left[e_{\alpha}, e_{\mu}\right]^{\bar{\nu}}=-\partial_{\mu} c_{\alpha}{ }^{\bar{\nu}}, \\
& 0=\left[e_{\alpha}, e_{\beta}\right]^{\bar{\nu}}=c_{\alpha}{ }^{\bar{\nu}}, \beta-c_{\beta}{ }^{\bar{\nu}}, \alpha+c_{\alpha}{ }^{\bar{\rho}} c_{\beta}{ }^{\bar{\nu}}, \bar{\rho}-c_{\beta}{ }^{\bar{\rho}} c_{\alpha}{ }^{\bar{\nu}}, \bar{\rho},
\end{aligned}
$$

where, in writing the second equation, we have omitted terms that vanish as a consequence of the first equation.

Furthermore, both $\mathbb{J}$ and $\pi$ are elements of $\operatorname{End}\left(\mathcal{T}_{\boldsymbol{Z}}\right)$. As their action commutes they can be diagonalised simultaneously, inducing a further split

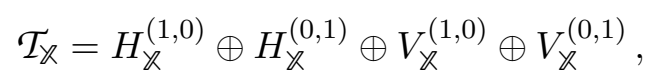

which is realised through

$$
H_{\mathbb{K}}^{(1,0)}=\operatorname{span}\left\{e_{\alpha}=\partial_{\alpha}-c_{\alpha}{ }^{\mu} \partial_{\mu}\right\}, \quad V_{\mathbb{K}}^{(1,0)}=\operatorname{span}\left\{\partial_{\mu}\right\},
$$

together with their complex conjugates. In fact, in the first line one could have included a mixed term $-c_{\alpha}{ }^{\bar{\nu}} \partial_{\bar{\nu}}$. This vanishes because the following relation needs to hold if $\mathbb{X}$ is complex

$$
\operatorname{span}\left\{e_{\alpha}, \partial_{\mu}\right\}=\operatorname{span}\left\{\partial_{\alpha}, \partial_{\mu}\right\}
$$


Note that if

$$
c_{\alpha}{ }^{\bar{\nu}}=0,
$$

then equations (2.24) are both satisfied, without further conditions on the $c_{a}{ }^{m}$.

We can decompose $Đ$ and $\mathrm{Ð}^{\sharp}$ into holomorphic type

$$
\begin{aligned}
Ð & =\check{\partial}+\bar{\partial}, \\
\bigoplus^{\sharp} & =\check{\partial}^{\sharp}+\overline{\varpi^{\sharp}}=\mathrm{d} y^{\alpha} \bigoplus_{\alpha}^{\sharp}+\mathrm{d} y^{\bar{\beta}} \overline{\mathrm{Ð}^{\sharp}},
\end{aligned}
$$

where $ð, \bar{\partial}$ both square to zero and anticommute, and are Dolbeault operators with respect to $J$. That is, $\bar{\partial}$ maps a $J-(p, q)$ form to a $J-(p, q+1)$-form. Their close relatives, $\partial^{\sharp}$ and $\overline{\partial^{\sharp}}$ anticommute when $S_{\alpha \bar{\beta}}{ }^{m}=0$ and $\overline{\partial^{\sharp}}$ squares to zero when $S_{\bar{\alpha} \bar{\beta}} m=0$. When this is the case $\overline{\partial^{\sharp}}$ is a Dolbeault operator with respect to $J^{\sharp}$ mapping a $J^{\sharp}-(p, q)$ form to a $J^{\sharp}-(p+1, q)$ form.

Recall that in special geometry and Kodaira-Spencer theory, the parameter variation of complex structure and the integrability condition $N_{J}=0$, to first order, becomes

$$
\Delta_{\alpha}{ }^{\mu} \in H_{\bar{\partial}}^{(0,1)}\left(X, \mathcal{T}_{X}^{(1,0)}\right) .
$$

The covariant deformation of the holomorphic projector $P_{m}{ }^{n}=\frac{1}{2}\left(\delta_{m}{ }^{n}-\mathrm{i} J_{m}{ }^{n}\right)$ is given by the Lie derivative (2.20), and this facilitates a covariant definition of $\Delta_{\alpha}$ :

$$
\Delta_{a}=\mathcal{L}_{e_{a}} P=\left(e_{a}\left(P_{n}{ }^{m}\right)+\left(\partial_{k} c_{a}{ }^{m}\right) P_{n}{ }^{k}-\left(\partial_{n} c_{a}{ }^{k}\right) P_{k}{ }^{m}\right) e^{n} \otimes \partial_{m} .
$$

If $N_{\mathbb{L}}=0$ so that the product structure is integrable, then we can find a set of adapted coordinates in which $c_{a}{ }^{m}=0$ and that $\Delta_{a}=\partial_{a} P_{m}{ }^{n} \mathrm{~d} x^{m} \partial_{n}$. This is what is familiar from special geometry. Provided $\partial_{a} J_{m}{ }^{n} \neq 0$, so that complex structure depends on parameters, we cannot find a set of holomorphic coordinates in which $c_{a}$ is also zero.

On the other hand, as $N_{\S}=0$ we can find a set of holomorphic coordinates in which $\sqrt{ }$ is constant and diagonal. In that case

$$
\mathcal{L}_{e_{\alpha}} P_{\bar{\nu}}{ }^{\mu}=-\partial_{\bar{\nu}} c_{\alpha}{ }^{\mu} .
$$

In this coordinate chart we identify

$$
-\overline{\mathrm{\partial}} c_{\alpha}=\Delta_{\alpha}{ }^{\mu} \partial_{\mu}
$$

Although the symbols $c_{\alpha}{ }^{\mu}$ transform in the manner of a connection, the quantity $\partial_{\bar{\nu}} c_{\alpha}{ }^{\mu}$ is covariant under holomorphisms and so is a well-defined $\bar{\varnothing}$-closed form, consistent with (2.26). It is important to note that we cannot set $c_{\alpha}{ }^{\mu}=0$ since $\Delta_{\alpha}$ is a tensor which does not vanish.

If we have $N_{\mathbb{L}}=N_{\S}=0$ then this does not necessarily imply we can find a set of holomorphic coordinates for $\mathbb{X}$ in which $c$ vanishes. Indeed, as we see from the above $\mathcal{L}_{a} J=\left[e_{a}, J\right]_{F N}$ is precisely the obstruction to doing this. Instead, the structures $\mathbb{J}$ and $\mathbb{L}$ are simultaneously integrable if

$$
[\mathbb{J}, \mathbb{l}]_{F N}=0, \quad[\mathbb{L}, \mathbb{L}]_{F N}=0, \quad[\mathbb{J}, \mathbb{L}]_{F N}=0 .
$$


While we will often use the first two conditions, we will always have $[\mathbb{J}, \mathbb{L}]_{F N}$ being nonvanishing and so we cannot discard $c$.

In $[11,12]$ it is observed that given $\left\{e_{\alpha}\right\}$ define normal vectors to fibres $c_{\alpha}{ }^{\mu}$ with respect to the metric on $\mathcal{X}$. Hence, $c_{\alpha}{ }^{\mu}$ is related to the extrinsic curvature by $\left(\partial_{\bar{\mu}} c_{\alpha}{ }^{\rho}\right) g_{\rho \bar{\nu}}$ which describes the curvature of fibres are embedded within $\mathbf{X}$. It is also shown that deformations of complex structure satisfy exactly the relation (2.29). We discuss this further below.

The $\mathbb{J}$-Dolbeault operator $\mathbb{D}$ acting on a form of $\mathbb{J}$-type $(p, q)$, denoted $\mathbb{W}^{(p, q)}$, is defined as

$$
\partial \mathbb{W}^{(p, q)}=(\mathbb{d} \mathbb{W})^{(p+1, q)} .
$$

In the language of $\mathbb{X}, \partial_{\alpha}$ is promoted to a Lie derivative $\mathcal{L}_{e_{\alpha}}$ while holomorphic type of vertical forms are defined by the projectors

$$
P=\frac{1}{2}\left(\delta_{m}^{n}-\mathrm{i}{J_{m}}^{n}\right) e^{m} \otimes \partial_{n}, \quad \text { and } \quad Q=\frac{1}{2}\left(\delta_{m}{ }^{n}+\mathrm{i}{J_{m}}^{n}\right) e^{m} \otimes \partial_{n} .
$$

We have $\mathcal{L}_{e_{\alpha}} P=\Delta_{\alpha}$ and $\mathcal{L}_{e_{\alpha}} Q=-\Delta_{\alpha}$. On a corporal 1-form $\eta=\eta_{m} e^{m}$, the $\bigoplus_{\alpha}^{\sharp}$ operator is the appropriate projection of the Lie derivative:

$$
\bigoplus_{\alpha}^{\sharp} \eta^{(p, q)}=\left(\mathcal{L}_{e_{\alpha}} \eta\right)^{(p, q)} \text {. }
$$

This is precisely the holotypical derivative denoted by $\mathcal{D}_{\alpha}$ in [1]. As may be seen by writing out the components for a 1-form, for example

$$
\begin{aligned}
& \bigoplus_{\alpha}^{\sharp} \eta^{(1,0)}=\left(\mathcal{L}_{e_{\alpha}} \eta_{m}\right) P_{n}{ }^{m} e^{n}=\mathcal{L}_{e_{\alpha}}\left(P_{n}{ }^{m} \eta_{m} e^{n}\right)-\Delta_{\alpha}{ }^{m} \eta_{m}, \\
& \bigoplus_{\alpha}^{\sharp} \eta^{(0,1)}=\left(\mathcal{L}_{e_{\alpha}} \eta_{m}\right) Q_{n}{ }^{m} e^{n}=\mathcal{L}_{e_{\alpha}}\left(Q_{n}{ }^{m} \eta_{m} e^{n}\right)+\Delta_{\alpha}{ }^{m} \eta_{m} .
\end{aligned}
$$

The second equality follows by the Leibniz rule.

\subsection{The connection $c_{a}{ }^{m}$ as the shift, and the extrinsic curvature of $X_{y}$}

Recall first the formalism relating to the extrinsic curvature of a submanifold as it applies in an elementary setting such as in figure 1 . Here we have a curve which we approximate to second order by a circle of curvature. At a point on the curve we have a normal $\delta \mathbf{n}=\delta r \mathbf{n}$ $(\mathbf{n}=\partial / \partial r)$ and a tangent $\mathbf{m}(=\partial / \partial \theta)$. We consider the result of parallely propagating $\delta \mathbf{n}$, in the embedding metric, to a nearby point, this gives the dashed vector in the figure. The pre-existing normal $\delta \mathbf{n}$ at the displaced point differs from this by an amount proportional to $\mathbf{m}$. We write ${ }^{5}$

$$
\mathbf{m} \cdot \nabla \mathbf{n}=\chi \mathbf{m}
$$

and this defines the extrinsic curvature $\chi$. Either from the diagram, or from a direct calculation of the covariant derivative, one sees that $\chi=1 / r$ for the situation depicted.

We could study also the variation of the tangent vector, by parallely propagating the tangent vector to a nearby point on the surface and comparing it with the preexisting tangent vector there. In this way we see that

$$
\mathbf{m} \cdot \nabla \mathbf{m}=-\chi \mathbf{n} .
$$

\footnotetext{
${ }^{5}$ There is a choice of sign here. Our choice makes the extrinsic curvature of a cylinder in a flat embedding space positive. An opposite convention is also common.
} 
Again, we can check this directly by computing the covariant derivative, and we can also deduce this relation by noting that the right hand side is in the direction of $-\mathbf{n}$ and the coefficient follows from the previous relation, on noting that $\mathbf{m} \cdot \nabla(\mathbf{m} \cdot \mathbf{n})=0$.

We turn now to figure 2 , which relates to the fibration $\mathcal{X}$. We first consider the case that the quantity $c_{a}{ }^{m}$ vanishes, so that the normals connect the points labeled by $x$ on $X_{y}$ and $X_{y+\delta y}$ and also the points labelled by $x+\delta x$. We may parallely propagate the normal $e_{a}$ from $x$ to $x+\delta x$, on $X_{y}$, and compare it with the preexisting normal there. In this way we can define and extrinsic curvature tensor $\chi_{a m}{ }^{n}$

$$
e_{m} \cdot \nabla e_{a}=\chi_{a m}^{n} e_{n}
$$

We can study the variation of the tangents rather than the variation of the normals. We take a tangent vector $e_{n}$ at $x$, parallely propagate it to $x+\delta x$ and compare it with the pre-existing tangents. There will be an out of surface component that can be expressed in terms of the normal vectors. This process yields

$$
e_{m} \cdot \nabla e_{n}=-\chi_{m n}^{a} e_{a}+\mathbb{\nabla}_{m}{ }_{n}^{k} e_{k} .
$$

The fact that the coefficients that involve the $e_{a}$, on the right hand side, are the extrinsic curvature follows from (2.32), on noting that $e_{m} \cdot \nabla_{\mathfrak{g}}\left(e_{a}, e_{n}\right)=0$. We see also, in this way, that the $a$ index on the extrinsic curvature is raised and lowered with the metric $g_{a b}$, while the $n$ index is raised and lowered with the metric $g_{m n}$.

The extrinsic curvature so defined is a tensor, so covariant and so unaffected by whether we choose to take $c_{a}{ }^{m}=0$. However, if we do so, we can identify the extrinsic curvature with minus the Christoffel symbol $\mathbb{\varpi}_{m}{ }^{n}{ }_{a}$.

Now let us include the effect of nonzero $c_{a}{ }^{m}$ and turn to figure 3. The vector $\delta y^{\alpha} \partial_{\alpha}$ now connects the two points $(y, x)$ and $(y+\delta y, x)$. So the point labeled by $x$ on $X_{y}$ with the point labeled by $x$ on $X_{y+\delta y}$. The normal vector $\delta y^{\alpha} e_{\alpha}$ connects the point $x$ on $X_{y}$ with the point $x-c_{\alpha} \delta y^{\alpha}$ on $X_{y+\delta y}$. The difference is the vertical vector $c_{\alpha} \delta y^{\alpha}$. Following the usage in relativity, we refer to $c_{\alpha}$ as the shift. For the displaced point $x+\delta x$, the shift has become $\left(c_{\alpha}+\delta c_{\alpha}\right) \delta y^{\alpha}$. For the case of real coordinates, we have the freedom to take $c_{a}{ }^{m}=0$. In complex coordinates, however, this is no longer possible. Indeed the shift plays an essential role.

We see from (2.32) that the extrinsic curvature is a rotation coefficient

$$
\chi_{a m}{ }^{n}=\mathbb{\Theta}_{a}{ }^{n}{ }_{m}=\frac{1}{2} g^{n k} \mathfrak{D}_{a} g_{k m},
$$

where $\Theta$ are the rotation coefficients in the $e$-basis, and the last term follows from computing the Levi-Civita connection coefficient in the $e$-basis, see appendix B.1, with

$$
\mathfrak{D}_{a} g_{k m}=e_{a}\left(g_{k m}\right)-c_{a}{ }^{\ell}, k g_{\ell m}-c_{a}^{\ell},{ }_{m} g_{\ell k} .
$$

In complex coordinates, it follows that we have

$$
\chi_{\alpha \bar{\mu}}{ }^{\nu}=\frac{1}{2} g^{\nu \bar{\kappa}} \mathfrak{D}_{\alpha} g_{\overline{\kappa \mu}}=-\frac{1}{2} g^{\nu \bar{\lambda}}\left(c_{\alpha}{ }^{\rho}, \bar{\lambda} g_{\rho \bar{\mu}}+c_{\alpha}{ }^{\rho}, \bar{\mu} g_{\rho \bar{\lambda}}\right) .
$$




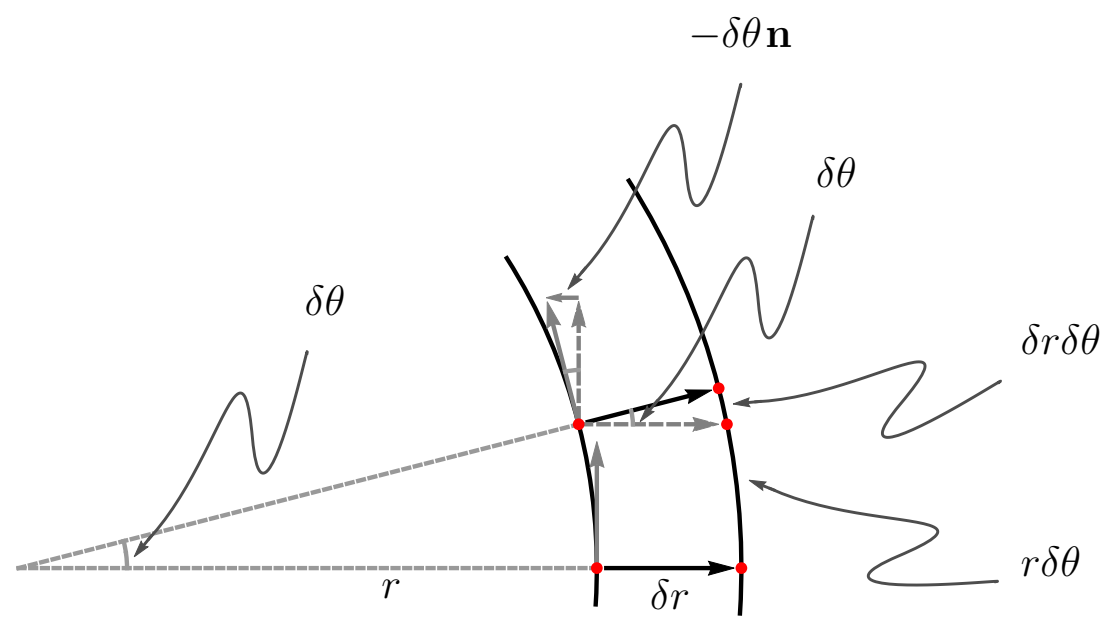

Figure 1. The calculation of the extrinsic curvature for a cylinder embedded in a flat space.

The last term in this equation expresses the extrinsic curvature in terms of the derivatives of the shift.

We also know from (2.29) that $c_{\alpha}{ }^{\rho}, \bar{\lambda}=-\Delta_{\alpha \bar{\lambda}^{\rho}}$. So we also have the following expression for the extrinsic curvature in terms of $\Delta_{\alpha}{ }^{\nu}$

$$
\chi_{\alpha \overline{\mu \nu}}=\Delta_{\alpha(\overline{\mu \nu})} \cdot
$$

The extrinsic curvature is a tensor, as is $\Delta_{\alpha(\overline{\mu \nu})}$, so we see from (2.33) that we cannot set $c_{\alpha}{ }^{\nu}=0$. In fact the extrinsic curvature is the obstruction to so doing. The reason that this is so, is that the shift is defined in terms of the normal to the fibres, and so by the metric $\mathbb{g}_{m n}$, thus $c_{\alpha}{ }^{\nu}$ is not a holomorphic function of the coordinates and so cannot be removed by a holomorphic coordinate transformation.

\section{The universal geometry $\mathcal{U}$}

In the previous sections we have described in detail how to extend the geometry of $X$ to the larger structure of the fibration $\mathfrak{X}$. This also allowed us to describe geometrically the variations of the metric and complex structures on $X$ in terms of Lie derivatives and flows on the moduli space $M$. We now study the geometry $\mathcal{U}$, the universal bundle, whose base manifold is $\mathbb{X}$. This is a holomorphic bundle with connection $\mathbb{A}$, with $\mathbb{A}$ the natural extension of $A$. The field strength $\mathbb{F}$ for $\mathbb{A}$ has a tangibility $[1,1]$ part which exactly describes the variation of $A$. The Bianchi identity for $\mathbb{E}$ efficiently encapsulates otherwise subtle identities derived in [1].

The universal geometry also includes the three-form $H=\mathrm{d}^{c} \omega$ and its Bianchi identity (1.2). The extension of $H$ to $\mathbb{X}$ is defined in a natural way

$$
\mathbb{H}=\mathbb{d}^{c} \omega .
$$

We demand that $\mathbb{H}$ obeys an extended Bianchi identity

$$
\mathbb{d} \mathbb{H}=-\frac{\alpha^{\prime}}{4}\left(\operatorname{Tr}\left(\mathbb{F}^{2}\right)-\operatorname{Tr}\left(\mathbb{R}^{2}\right)\right) .
$$




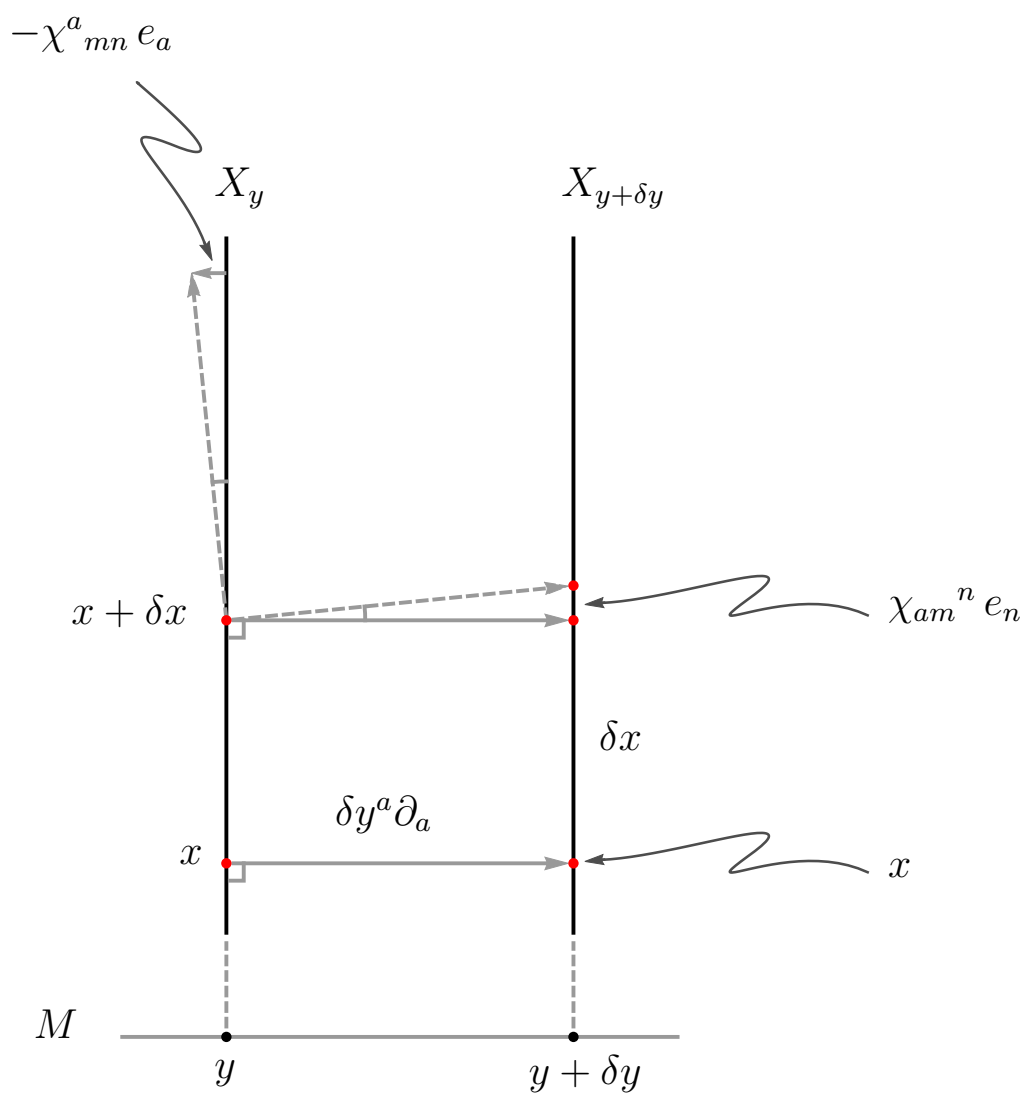

Figure 2. The calculation of the extrinsic curvature for the fibres $X_{y}$ for the case that $c_{a}{ }^{m}=0$.

Remarkably, this equation elegantly captures otherwise complicated algebraic relations derived with much effort in [1]. These identities are important as they are central to the construction of the metric on $M$ and showing that it is Kähler. Using the extended quantities on $\mathbb{X}$ we re-derive the metric on $M$ in a concise fashion in section 5 .

\subsection{The extension of $A$}

The covariant derivative for $A$ defined in [1] transforms covariantly under gauge transformations. It needs to be generalised to transform, additionally, under bundle diffeomorphisms (2.10). To do this we define an extended connection $\mathbb{A}$ for the extended vector bundle $\mathcal{U} \rightarrow \mathbb{X}$

$$
\mathbb{A}=A_{m} e^{m}+A_{a}^{\sharp} \mathrm{d} y^{a}, \quad A_{a}^{\sharp}=\Lambda_{a}-A_{m} c_{a}^{m},
$$

where the components of the corpus $A_{m}$ are identified with the connection along $X$. In the following, we will denote the corpus of $\mathbb{A}$ by $A=A_{m} e^{m}$ in the e-basis, the animus by $A^{\sharp}=A_{a}^{\sharp} \mathrm{d} y^{a}$. We can divide the form into holomorphic type

$$
\mathbb{A}=\mathcal{A}-\mathcal{A}^{\dagger}, \quad \mathcal{A}=\mathbb{A}^{(0,1)} .
$$

We will not be specific about the structure group of the universal bundle $\mathcal{U}$ beyond requiring it contain $\mathfrak{G}$ as a subgroup when restricted to $X$ appropriately. This restriction is important in later sections when we discuss deformations of $\mathcal{T}_{X}$. 


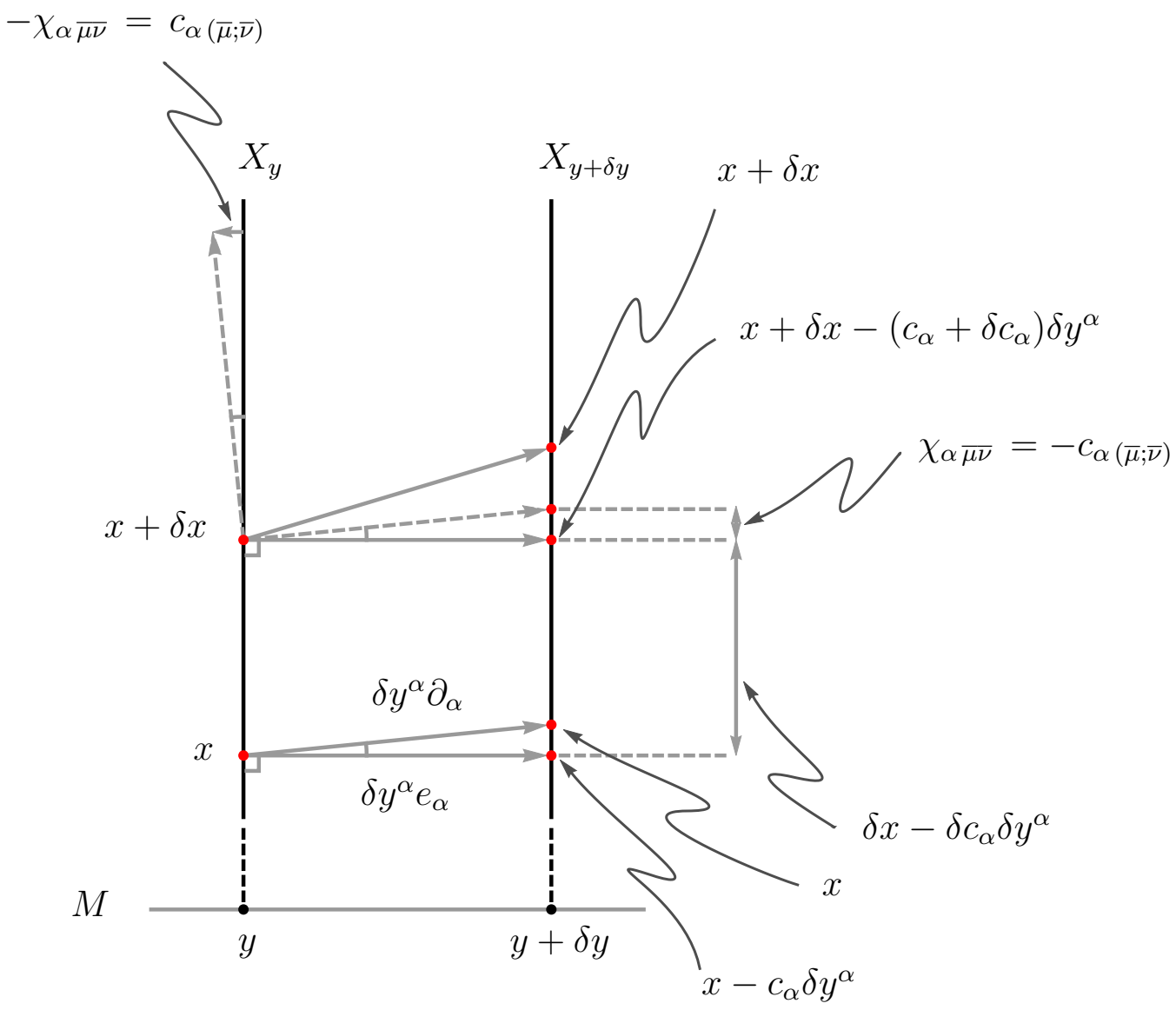

Figure 3. The calculation of the extrinsic curvature for the fibres $X_{y}$ for the case of complex coordinates.

The form $\mathcal{A}$ can be decomposed into its animus and corpus

$$
\mathcal{A}=\mathcal{A}_{\bar{\alpha}}^{\sharp} \mathrm{d} y^{\bar{\alpha}}+\mathcal{A}_{\bar{\mu}} e^{\bar{\mu}} .
$$

The field strength of $\mathbb{A}$ is defined as usual

$$
\mathbb{F}=\mathbb{d} \mathbb{A}+\mathbb{A}^{2} .
$$

This can be decomposed according to tangibility and in terms of the covariant derivatives $Đ, \bigoplus^{\sharp}$, defined in (2.15) and (2.16), respectively:

$$
\mathbb{F}=\left(Đ+\bigoplus^{\sharp}-S\right)\left(A+A^{\sharp}\right)+\left(A+A^{\sharp}\right)^{2}=\frac{1}{2} F_{m n} e^{m} e^{n}+\mathrm{d} y^{a} \mathbb{F}_{a}^{\sharp}+\frac{1}{2} \mathbb{F}_{a b}^{\sharp} \mathrm{d} y^{a} \mathrm{~d} y^{b} .
$$

Let us unpackage each of the three components of $\mathbb{F}$. The corpus is the field strength of $A$ on $X$,

$$
F_{m n}=\partial_{m} A_{n}-\partial_{n} A_{m}+A_{m} A_{n}-A_{n} A_{m} .
$$

The second term defines a covariant derivative that transforms homogeneously under gauge transformations and is invariant under bundle diffeomorphisms:

$$
\mathbb{F}_{a}^{\sharp}=\mathfrak{D}_{a} A, \quad \text { where } \mathfrak{D}_{a} A=e_{a}(A)-\left(\mathrm{Đ} c_{a}{ }^{m}\right) A_{m}-\mathrm{Ð}_{A} A_{a}^{\sharp},
$$


here

$$
\mathrm{Ð}_{A} A_{a}^{\sharp}=\mathrm{Đ} A_{a}^{\sharp}+\left[A, A_{a}^{\sharp}\right],
$$

and

$$
e_{a}(A)=\partial_{a} A-c_{a}^{m} \partial_{m} A .
$$

On a gauge neutral object, $\mathfrak{D}_{a}$ reduces to $\bigoplus_{a}^{\sharp}$.

In holomorphic coordinates, using the identification of $\Delta_{\alpha}$ in (2.29), we find it is the appropriate generalisation of the holotypical derivative introduced in [1]:

$$
\mathfrak{D}_{\alpha} \mathcal{A}=e_{\alpha}(\mathcal{A})-\Delta_{\alpha}{ }^{\nu} \mathcal{A}_{\nu}^{\dagger}-\overline{\mathrm{\partial}}_{\mathcal{A}} A_{\alpha}^{\sharp} .
$$

The third equation of (3.3) is

$$
\mathbb{F}_{a b}^{\sharp}=2 \bigoplus_{[a}^{\sharp} A_{b]}^{\sharp}+\left[A_{a}^{\sharp}, A_{b}^{\sharp}\right]-S_{a b}{ }^{m} A_{m}, \quad \text { where } \bigoplus_{a}^{\sharp} A_{b}^{\sharp}=e_{a}\left(A_{b}^{\sharp}\right) .
$$

We take $\mathcal{U}$ to be holomorphic meaning

$$
\mathbb{F}^{(0,2)}=0 .
$$

The corpus of $\mathbb{F}$ automatically satisfies this requirement in virtue of $F^{(0,2)}=0$. The tangibility $[1,1]$ component is the condition that $\mathcal{A}$ depend holomorphically on parameters

$$
\mathfrak{D}_{\bar{\beta}} \mathcal{A}=0 .
$$

The tangibility $[2,0]$ component implies $\mathbb{F}_{\bar{\alpha} \bar{\beta}}^{\sharp}=0$. That is, that the bundle $\mathcal{U}$ restricted to $M$ is holomorphic. In deducing this we have used $S_{\bar{\alpha} \bar{\beta}}=0$.

Consider now the Bianchi identity for $\mathbb{E}$

$$
\mathbb{d}_{A} \mathbb{E}=0 .
$$

The corpus realises the Bianchi identity on $X$. The animus gives two further identities

$$
\mathrm{Ð}_{A}\left(\mathfrak{D}_{a} A\right)=\mathfrak{D}_{a} F \quad \text { and } \quad\left[\mathfrak{D}_{a}, \mathfrak{D}_{b}\right] A=-\mathrm{Ð}_{A}\left(\mathbb{F}_{a b}^{\sharp}\right)+S_{a b}{ }^{m} F_{m}=0,
$$

where

$$
\begin{aligned}
& \mathrm{Ð}_{A}\left(\mathfrak{D}_{a} A\right)=\mathrm{Ð}\left(\mathfrak{D}_{a} A\right)+\left[A, \mathfrak{D}_{a} A\right], \\
& \mathfrak{D}_{a} F=\bigoplus_{a}^{\sharp} F+\left[A_{a}^{\sharp}, F\right], \\
& \mathfrak{D}_{a}\left(\mathfrak{D}_{b} A\right)=\mathrm{Ð}_{a}^{\sharp}\left(\mathfrak{D}_{b} A\right)+\left[A_{a}^{\sharp}, \mathfrak{D}_{b} A\right], \\
& \mathrm{Ð}_{A} \mathbb{F}_{a b}^{\sharp}=Đ \mathbb{F}_{a b}^{\sharp}+\left[A, \mathbb{F}_{a b}^{\sharp}\right] \text {. }
\end{aligned}
$$

The relations (3.6) can be derived directly from the definition of the covariant derivative as in [1] with some labour. What we see here is an alternative derivation through the Bianchi identity. This also has the advantage of unification, reducing a pair of identities to a single identity.

The Atiyah constraint comes from taking $a=\alpha$ in the first equation of (3.6), and considering the $(0,2)$-component together with the identification of $\Delta_{\alpha}$ in (2.29):

$$
\overline{\mathrm{\partial}}_{\mathcal{A}}\left(\mathfrak{D}_{\alpha} \mathcal{A}\right)=\Delta_{\alpha}{ }^{\mu} F_{\mu} .
$$




\subsection{The extension of $B$ and $H$}

The field $\mathbb{H}$ is the extension of $H$, and defined as

$$
\mathbb{H}=\mathbb{d} \mathbb{B}-\frac{\alpha^{\prime}}{4}(\mathrm{CS}[\mathbb{A}]-\operatorname{CS}[\Theta]), \quad \text { where } \quad \operatorname{CS}[\mathbb{A}]=\operatorname{Tr}\left(\mathbb{A} \mathbb{d} A+\frac{2}{3} \mathbb{A}^{3}\right),
$$

where $\mathbb{B}$ is the extension of the Kalb-Ramond field

$$
\mathbb{B}=\frac{1}{2} B_{m n} e^{m} e^{n}+\mathbb{B}_{a m}^{\sharp} \mathrm{d} y^{a} e^{m}+\frac{1}{2} \mathbb{B}_{a b}^{\sharp} \mathrm{d} y^{a} \mathrm{~d} y^{b}=B+\mathbb{B}_{a}^{\sharp} \mathrm{d} y^{a}+B^{\sharp} .
$$

H decomposes as

$$
\mathbb{H}=\frac{1}{3 !} \mathrm{d} y^{a b c} \mathbb{H}_{a b c}^{\sharp}+\frac{1}{2} \mathrm{~d} y^{a b} \mathbb{H}_{a b}^{\sharp}+\mathrm{d} y^{a} \mathbb{H}_{a}^{\sharp}+H,
$$

where the $[1,2]$ term will be relevant in what follows. It is given by

$$
\mathbb{H}_{a}^{\sharp}=\bigoplus_{a}^{\sharp} B-Ð \mathbb{B}_{a}^{\sharp}-\frac{\alpha^{\prime}}{4}\left(\operatorname{Tr}\left(A_{a}^{\sharp} Đ A\right)-\operatorname{Tr}\left(\Theta_{a}^{\sharp} Đ \Theta\right)\right)+\frac{\alpha^{\prime}}{4}\left(\operatorname{Tr}\left(A \mathfrak{D}_{a} A\right)-\operatorname{Tr}\left(\Theta \mathfrak{D}_{a} \Theta\right)\right) .
$$

We can now rewrite this in terms of covariant derivatives

$$
\uplus_{a}^{\sharp}=\mathfrak{D}_{a} B+\frac{\alpha^{\prime}}{4}\left(\operatorname{Tr}\left(A \mathfrak{D}_{a} A\right)-\operatorname{Tr}\left(\Theta \mathfrak{D}_{a} \Theta\right)\right)-Ð \mathbb{B}_{a}^{\sharp},
$$

with the covariant derivative $\mathfrak{D}_{a} B$ is defined as

$$
\mathfrak{D}_{a} B=\bigoplus_{a}^{\sharp} B-\frac{\alpha^{\prime}}{4}\left(\operatorname{Tr}\left(A_{a}^{\sharp} Đ A\right)-\operatorname{Tr}\left(\Theta_{a}^{\sharp} Đ \Theta\right)\right),
$$

which sharpens the relation derived in [1]. We will see why this is a covariant derivative shortly.

By demanding $\mathbb{H}$ be gauge invariant, we see that the field $\mathbb{B}$ transforms under gauge transformations:

$$
\mathbb{B} \rightarrow{ }^{\Phi, \Psi} \mathbb{B}=\mathbb{B}+\frac{\alpha^{\prime}}{4}\{\operatorname{Tr}(\mathbb{Y} \mathbb{A}-\mathbb{Z} \Theta)+\mathbb{U}-\mathbb{W}\} .
$$

Which is the natural extension of the rule given in (1.13). Given the above relations the field strength $\mathbb{H}$ is invariant. As the animus of $\mathbb{B}$ transforms inhomogeneously, it is inconsistent to try to set it to zero. Here $\mathbb{Y}, \mathbb{U}$ are the extensions of $Y$ and $U$ :

$$
\mathbb{Y}=\Phi^{-1} \mathbb{d} \Phi, \quad \mathbb{d} \mathbb{U}=\frac{1}{3} \operatorname{Tr} \Upsilon^{3},
$$

with $\mathbb{Z}, \mathbb{W}$ being the spin connection counterpart.

The right hand side of (3.9) is the combination of terms identified in [1] as being gauge invariant. This we now understand since $\mathcal{B}_{a}=\mathbb{H}_{a}^{\sharp}$ and $\mathbb{H}$ is gauge invariant.

The covariant derivative is defined such that it transforms in a manner parallel to the $B$-field itself:

$$
{ }^{(\Phi, \Psi)} \mathfrak{D}_{a} B=\mathfrak{D}_{a} B+\frac{\alpha^{\prime}}{4}\left(\operatorname{Tr}\left(Y \mathfrak{D}_{a} A\right)+\mathfrak{Y}_{a}-\operatorname{Tr}\left(Z \mathfrak{D}_{a} \Theta\right)-\mathfrak{Z}_{a}\right) .
$$


We have also defined

$$
\begin{aligned}
& \mathfrak{Y}_{a}=\bigoplus_{a}^{\sharp} U-\operatorname{Tr}\left(Y_{a}^{\sharp} Y^{2}\right)+Đ\left(\operatorname{Tr}\left(Y_{a}^{\sharp} A-A Y_{a}^{\sharp}\right)\right), \\
& \mathfrak{Z}_{a}=\bigoplus_{a}^{\sharp} Z-\operatorname{Tr}\left(Z_{a}^{\sharp} Z^{2}\right)+Ð\left(\operatorname{Tr}\left(Z_{a}^{\sharp} \Theta-\Theta_{a}^{\sharp} Z\right)\right),
\end{aligned}
$$

Using that the form $\mathbb{Y}$ satisfies $\mathbb{d} Y=-\mathbb{Y}^{2}$, we find that this quantity is Đ-closed

$$
\mathrm{Đ} \mathfrak{Y}_{a}=0 \text {. }
$$

In addition to the gauge transformations above the field strength $\mathbb{H}$ is invariant under an additional symmetry, in which $\mathbb{B}$ shifts by a $\mathbb{d}$-exact amount,

$$
\mathbb{B} \rightarrow \mathbb{B}+\mathbb{d} \beta, \quad \mathbb{\beta}=\beta_{m} e^{m}+\beta_{a}^{\sharp} \mathrm{d} y^{a},
$$

where the one-form $\beta$ is gauge-invariant. Decomposing this into tangibilities we have

$$
\begin{aligned}
B & \rightarrow B+Đ \beta, \\
\mathbb{B}_{a}^{\sharp} & \rightarrow \mathbb{B}_{a}^{\sharp}+\bigoplus_{a}^{\sharp} \beta-Ð \beta_{a}^{\sharp}, \\
\mathbb{B}_{a b}^{\sharp} & \rightarrow \mathbb{B}_{a b}^{\sharp}+\bigoplus_{a}^{\sharp} \beta_{b}^{\sharp}-\bigoplus_{b}^{\sharp} \beta_{a}^{\sharp}-S_{a b}{ }^{m} \beta_{m} .
\end{aligned}
$$

The first line corresponds to shifting $B$ by a $Đ$-exact term. The second line corresponds to shifts of $\mathbb{B}_{a}^{\sharp}$. The way to think of $\mathbb{B}_{a}^{\sharp}$ is that it is another connection; its purpose to is define an invariant quantity $\mathcal{B}_{a}$ as in (3.9). This invariance can be checked directly, but an easier way to see this is to note that $\mathbb{H}$ is invariant and so $\mathbb{H}_{a}^{\sharp}=\mathcal{B}_{a}$ is invariant. The quantity $\mathcal{B}_{a}$, mentioned in the introduction, plays an important role as $\mathcal{B}_{a}+\mathrm{i} \mathfrak{D}_{a} \omega$ plays the role in heterotic geometry analogous to the role of complexified Kähler class in special geometry. All this goes to show that the animi of $\mathbb{A}$ and $\mathbb{B}$ are connections which are needed to define covariant derivatives on the moduli space.

Although we have not fully explored this aspect, we believe the quantity $\mathbb{B}_{a b}^{\sharp}$ with the transformation rules as in the third line above, provide connections that enable one to define second and higher order derivatives. For example, see [1] where a second order covariant derivative was defined.

\subsection{The extension of $\mathrm{d}^{c} \omega$}

We will shortly have need for the quantity

$$
\mathbb{d}^{c} \omega=\frac{1}{3 !} \rrbracket^{P} \rrbracket^{Q} \rrbracket^{R}(\mathbb{d} \omega)_{P Q R} .
$$

In a holomorphic basis $\omega$ is $(1,1)$ and so

$$
\mathbb{d}^{c} \omega=\mathrm{i}(\mathbb{d} \omega)^{(2,1)}-\mathrm{i}(\mathbb{d} \omega)^{(1,2)} .
$$

The term $\mathbb{d}^{c} \omega$ has vanishing $[3,0]$ term due to the fact that $g_{\alpha \bar{\beta}}^{\sharp}$ is Kähler, while the remaining components are given by

$$
\begin{aligned}
& \left(\mathbb{d}^{c} \omega\right)_{\alpha}=\mathrm{i} \mathfrak{D}_{\alpha} \omega^{(1,1)}-\mathrm{i} \mathfrak{D}_{\alpha} \omega^{(0,2)}, \\
& \left(\mathbb{d}^{c} \omega\right)_{\alpha \beta}=-\mathrm{i} S_{\alpha \beta}{ }^{\mu} \omega_{\mu}, \quad\left(\mathbb{d}^{c} \omega\right)_{\bar{\alpha} \bar{\beta}}=\mathrm{i} S_{\bar{\alpha} \bar{\beta}}{ }^{\bar{\mu}} \omega_{\bar{\mu}}, \\
& \left(\mathbb{d}^{c} \omega\right)_{\alpha \bar{\beta}}=-\mathrm{i} S_{\alpha \bar{\beta}}{ }^{\bar{\mu}} \omega_{\bar{\mu}}+\mathrm{i} S_{\alpha \bar{\beta}^{\mu}} \omega_{\mu} .
\end{aligned}
$$


Note that the action of the covariant derivative $\mathfrak{D}_{\alpha}$ on a gauge neutral object is the same as $\bigoplus^{\sharp}$ so that $\mathfrak{D}_{\alpha} \omega^{(p, q)}=\bigoplus_{a}^{\sharp} \omega^{(p, q)}$. In the sections to follow, where no ambiguity will arise we will use $\mathfrak{D}_{\alpha}$ to prevent an unnecessary proliferation of symbols.

On setting $S=0$ the expression simplifies significantly

$$
\mathbb{d}^{c} \omega=\mathrm{i}(ð-\bar{\partial}) \omega+\mathrm{id} y^{\alpha}\left(\mathfrak{D}_{\alpha} \omega^{(1,1)}-\mathfrak{D}_{\alpha} \omega^{(0,2)}\right)+\mathrm{id} y^{\bar{\beta}}\left(\mathfrak{D}_{\bar{\beta}} \omega^{(2,0)}-\mathfrak{D}_{\bar{\beta}} \omega^{(1,1)}\right) .
$$

While $\omega$ is type $(1,1)$, its derivative $\mathfrak{D}_{\alpha}$ is type $(2,1) \oplus(1,2): \mathfrak{D}_{\alpha} \omega=\mathfrak{D}_{\alpha} \omega^{(1,1)}+\mathfrak{D}_{\alpha} \omega^{(0,2)}$, and this expresses the type changing property of variations with respect to complex structure.

\subsection{The relation $H=\mathbb{d}^{c} \omega$, Bianchi identity and second order relations}

We suppose that the extended supersymmetry relation (3.1) holds on $\mathbb{X}$ This imposes some constraints on the variations of a heterotic structure. The tangibility $[1,2]$ part of this relation gives

$$
\begin{aligned}
\mathcal{B}_{\alpha}{ }^{(2,0)} & =0, \\
\mathcal{B}_{\alpha}{ }^{(1,1)}-\mathrm{i} \mathfrak{D}_{\alpha} \omega^{(1,1)} & =0, \\
\mathcal{B}_{\alpha}{ }^{(0,2)}+\mathrm{i} \mathfrak{D}_{\alpha} \omega^{(0,2)} & =0 .
\end{aligned}
$$

We define

$$
Z_{\alpha}=\mathcal{B}_{\alpha}+\mathrm{i} \mathfrak{D}_{\alpha} \omega, \quad \text { and } \quad \bar{Z}_{\alpha}=\mathcal{B}_{\alpha}-\mathrm{i} \mathfrak{D}_{\alpha} \omega
$$

which are the generalisation to heterotic geometry of the variation of the complexified Kähler class familiar in special geometry $\delta B+\mathrm{i} \delta \omega$. In terms of $Z, \bar{Z},(3.13)$ can be written as

$$
\begin{aligned}
& Z_{\alpha}{ }^{(2,0)}=\bar{Z}_{\alpha}{ }^{(2,0)}=0, \\
& \bar{Z}_{\alpha}{ }^{(1,1)}=0, \\
& Z_{\alpha}{ }^{(0,2)}=0 .
\end{aligned}
$$

These equations described first order conditions on the heterotic moduli which were derived in [13-16] and in this notation in [1] by taking partial derivatives of the supersymmetry relation $H=\mathrm{d}^{c} \omega$. We identify $\mathbb{B}_{a}$ with $b_{a}$ and note that $\mathbb{H}=\mathbb{d}^{c} \omega$ captures all of the moduli equations except one. For the remaining one we turn to the Bianchi identity for $\mathbb{d} \mathbb{H}$ on $\mathbb{X}$ :

$$
\mathbb{d} \mathbb{H}=-\frac{\alpha^{\prime}}{4}\left(\operatorname{Tr} \mathbb{F}^{2}-\operatorname{Tr} \mathbb{R}^{2}\right)=\mathbb{d}\left(\mathbb{d}^{c} \omega\right) .
$$

The curvatures $\mathbb{F}$ and $\mathbb{R}$ are of type $(1,1)$ and so only the type $(2,2)$ part of this relation is non-vanishing.

We start with tangibility $[1,3]$, focusing on holomorphic variation with index $\alpha$. The first equality of the previous equation is

$$
(\mathbb{d} H)_{\alpha}=-\frac{\alpha^{\prime}}{2}\left(\operatorname{Tr}\left(\mathfrak{D}_{\alpha} \mathcal{A} F\right)-\operatorname{Tr}\left(\mathfrak{D}_{\alpha} \vartheta R\right)\right) .
$$


Meanwhile $\left(\mathbb{d d}^{c} \omega\right)_{\alpha}$ is simplified using

$$
\begin{aligned}
Ð\left(\mathbb{d}^{c} \omega\right)_{\alpha} & =\mathrm{i}\left(\mathfrak{D}_{\alpha} \omega^{1,1}-\mathfrak{D}_{\alpha} \omega^{0,2}\right), \\
\mathfrak{D}_{\alpha}\left(\mathrm{Ð}^{c} \omega\right) & =2 \mathrm{i} \Delta_{\alpha}{ }^{\mu}(ð \omega)_{\mu}-2 \mathrm{i} \precsim\left(\Delta_{\alpha}{ }^{\mu} \omega_{\mu}\right)+\mathrm{i}(ð-\bar{\jmath}) \mathfrak{D}_{\alpha} \omega,
\end{aligned}
$$

and by using (3.13) we get

$$
\bar{\partial}\left(Z_{\alpha}^{(1,1)}\right)=2 \mathrm{i} \Delta_{\alpha}{ }^{\mu}(ð \omega)_{\mu}+\frac{\alpha^{\prime}}{2}\left(\operatorname{Tr}\left(\mathfrak{D}_{\alpha} \mathcal{A} F\right)-\operatorname{Tr}\left(\mathfrak{D}_{\alpha} \vartheta R\right)\right) .
$$

Let us now turn our attention to tangibility $[2,2]$. Assuming that $S=0$, this consists of two relations

$$
\begin{aligned}
\mathfrak{D}_{\alpha}\left(\mathbb{d}^{c} \omega\right)_{\beta}-\mathfrak{D}_{\beta}\left(\mathbb{d}^{c} \omega\right)_{\alpha}= & -\frac{\alpha^{\prime}}{2}\left(\operatorname{Tr}\left(\mathfrak{D}_{\alpha} \mathcal{A} \mathfrak{D}_{\beta} \mathcal{A}\right)-\operatorname{Tr}\left(\mathfrak{D}_{\alpha} \vartheta \mathfrak{D}_{\beta} \theta\right)\right), \\
\mathfrak{D}_{\alpha}\left(\mathbb{d}^{c} \omega\right)_{\bar{\beta}}-\mathfrak{D}_{\bar{\beta}}\left(\mathbb{d}^{c} \omega\right)_{\alpha}= & -\frac{\alpha^{\prime}}{2}\left(\operatorname{Tr}\left(\mathfrak{D}_{\alpha} \mathcal{A} \mathfrak{D}_{\bar{\beta}} \mathcal{A}^{\dagger}\right)-\operatorname{Tr}\left(\mathfrak{D}_{\alpha} \theta \mathfrak{D}_{\bar{\beta}} \theta^{\dagger}\right)\right) \\
& -\frac{\alpha^{\prime}}{4}\left(\operatorname{Tr}\left(\mathbb{F}_{\alpha \bar{\beta}}^{\sharp} F\right)-\operatorname{Tr}\left(\mathbb{R}_{\alpha \bar{\beta}}^{\sharp} R\right)\right) .
\end{aligned}
$$

The second relation forms part of a critical algebraic relation needed to derive the moduli space metric in [1] and so we focus on this one. It becomes

$$
\begin{aligned}
\mathfrak{D}_{\alpha}\left(\mathbb{d}^{c} \omega\right)_{\bar{\beta}}-\mathfrak{D}_{\bar{\beta}}\left(\mathbb{d}^{c} \omega\right)_{\alpha}=-2 \mathrm{i}\left(\mathfrak{D}_{\alpha} \mathfrak{D}_{\bar{\beta}} \omega\right)^{(1,1)}+2 \mathrm{i} \Delta_{\alpha}^{\mu}\left(\mathfrak{D}_{\bar{\beta}} \omega^{(2,0)}\right)_{\mu}+2 \mathrm{i} \Delta_{\bar{\beta}}^{\bar{\nu}}\left(\mathfrak{D}_{\alpha} \omega^{(0,2)}\right)_{\bar{\nu}} \\
+\mathrm{i} \mathfrak{D}_{\alpha}\left(\Delta_{\bar{\beta}}^{\bar{\nu}} \omega_{\bar{\nu}}\right)-\mathrm{i} \Delta_{\bar{\beta}}^{\bar{\nu}}\left(\mathfrak{D}_{\alpha} \omega^{(1,1)}\right)_{\bar{\nu}}+\mathrm{i} \mathfrak{D}_{\bar{\beta}}\left(\Delta_{\alpha}{ }^{\mu} \omega_{\mu}\right)-\mathrm{i} \Delta_{\alpha}{ }^{\mu}\left(\mathfrak{D}_{\bar{\beta}} \omega^{(1,1)}\right)_{\mu} .
\end{aligned}
$$

The last equation can be simplified by noticing a further relation

$$
\mathfrak{D}_{\alpha}\left(\Delta_{\bar{\beta}}^{\bar{\nu}} \omega_{\bar{\nu}}\right)-\Delta_{\bar{\beta}}^{\bar{\nu}}\left(\mathfrak{D}_{\alpha} \omega^{(1,1)}\right)_{\bar{\nu}}=\left(ð S_{\alpha \bar{\beta}}{ }^{\bar{\nu}}\right) \omega_{\bar{\nu}}=0,
$$

which sets the last line to zero. Putting everything together, we can rearrange (3.16) to obtain

$$
\begin{aligned}
\left(\mathfrak{D}_{\alpha} \mathfrak{D}_{\bar{\beta}} \omega\right)^{(1,1)}= & -\frac{\mathrm{i} \alpha^{\prime}}{4}\left(\operatorname{Tr}\left(\mathfrak{D}_{\alpha} \mathcal{A} \mathfrak{D}_{\bar{\beta}} \mathcal{A}^{\dagger}\right)-\operatorname{Tr}\left(\mathfrak{D}_{\alpha} \theta \mathfrak{D}_{\bar{\beta}} \theta^{\dagger}\right)\right) \\
& -\frac{\mathrm{i} \alpha^{\prime}}{8}\left(\operatorname{Tr}\left(\mathbb{F}_{\alpha \bar{\beta}}^{\sharp} F\right)-\operatorname{Tr}\left(\mathbb{R}_{\alpha \bar{\beta}}^{\sharp} R\right)\right)+\Delta_{\alpha}{ }^{\mu}\left(\mathfrak{D}_{\bar{\beta}} \omega^{(2,0)}\right)_{\mu}+\Delta_{\bar{\beta}}^{\bar{\nu}}\left(\mathfrak{D}_{\alpha} \omega^{(0,2)}\right)_{\bar{\nu}} .
\end{aligned}
$$

This shows that the Bianchi identity for $\mathbb{H}$ incorporates the second order algebraic relation for the variation of the hermitian form that is crucial in deriving the $\alpha^{\prime}$-corrected moduli metric.

\section{Using $\mathbb{X}$ to deform connections on $\mathcal{T}_{X}$}

The moduli space metric, expressed in (1.18), has a contribution from the variation of $\Theta$. As is the case for the gauge connection $A$, the variation of $\Theta$ is expressed as a covariant derivative with respect to parameters. However, unlike $A$, the derivatives of $\Theta$ are tied to the geometry of $X$, up to Lorentz gauge transformations. Our aim in this section is to compute $\mathfrak{D}_{\alpha} \Theta$ to zeroth order in $\alpha^{\prime}$, expressing the answer in terms of the moduli of $X$. 


\subsection{The covariant derivative of $\Theta$}

Our approach to computing covariant derivatives of $\Theta$ is to extend this connection to $\mathbb{X}$. The connection $\Theta$ is then a connection on the frame bundle, and its associated tangent bundle $\mathcal{T}_{\not{\chi}}$. We take the connection $\Theta$ to be metric compatible. It has a curvature two-form $\mathbb{R}=\mathbb{d} \Theta+\mathbb{\Theta}^{2}$ which transforms in a Lorentz algebra $\mathfrak{s o}(D)$ where $D=\operatorname{dim} \mathcal{X}$. In terms of tangibilities, $\Theta$ decomposes as

$$
\Theta=\Theta_{a}^{\sharp} \mathrm{d} y^{a}+\Theta_{m} e^{m} .
$$

The curvature $\mathbb{R}$ has a tangibility $[1,1]$ component which defines the covariant derivative

$$
\mathfrak{D}_{a} \Theta=\mathbb{R}_{a m}^{\sharp} e^{m} .
$$

For any frame on $\mathbb{X}$ with basis of sections $\left\{s_{a}, s_{m}\right\}$, the connection $\Theta$ has symbols

$$
\nabla s_{a}=\mathbb{\Theta}^{b}{ }_{a} s_{b}+\mathbb{\Theta}^{n}{ }_{a} s_{n}, \quad \nabla s_{m}=\mathbb{\Theta}^{n}{ }_{m} s_{n}+\mathbb{\Theta}^{b}{ }_{m} s_{b} .
$$

In the physical string theory, the connection $\Theta$ is $\mathfrak{s o}(6)$ valued, when $\left(s^{a}, s^{m}\right)$ form an orthonormal basis, and the term that appears in the moduli space metric involves a trace over $\mathfrak{s o}(6)$ :

$$
\operatorname{Tr}\left(\mathfrak{D}_{\alpha} \Theta \star \mathfrak{D}_{\bar{\beta}} \Theta\right)=\left(\mathfrak{D}_{\alpha} \Theta\right)^{m}{ }_{n} \star\left(\mathfrak{D}_{\bar{\beta}} \Theta\right)^{n}{ }_{m} .
$$

However, under a change of basis

$$
s_{a}=s_{a}^{b} e_{b}, \quad s_{m}=s^{n}{ }_{m} \partial_{n},
$$

for some invertible matrices $s^{b}{ }_{a}$ and $s^{n}{ }_{m}$. The covariant derivative $\mathfrak{D}_{a} \Theta$ transforms as

$$
\mathfrak{D}_{\alpha} \Theta^{m}{ }_{n} \rightarrow s^{m}{ }_{k} \mathfrak{D}_{\alpha} \Theta^{k}{ }_{l} s^{-1 l}{ }_{n},
$$

and so $\operatorname{Tr}\left(\mathfrak{D}_{\alpha} \Theta \star \mathfrak{D}_{\bar{\beta}} \Theta\right)$ is invariant. Hence, we are free to compute this term in the $e$-basis, which turns out to be very convenient. Our first task then is to compute the covariant derivative,

$$
\mathfrak{D}_{a} \Theta^{n}{ }_{m}=\bigoplus_{a}^{\sharp} \Theta^{n}{ }_{m}-Ð \Theta_{a}^{\sharp n}{ }_{m}+\left[\Theta_{a}^{\sharp}, \Theta\right]^{n}{ }_{m},
$$

in the $e$-basis. The first term in (4.3) is evaluated like a 1-form using (2.17). In explicit detail it is given by $\bigoplus_{a}^{\sharp} \Theta^{n}{ }_{m}=e_{a}\left(\Theta^{n}{ }_{m}\right)-e^{p}\left(\partial_{p} c_{a}{ }^{q}\right) \Theta_{q}{ }^{n}{ }_{m}$, while the second term is $Đ \Theta_{a m}^{\sharp n}=$ $e^{m} \partial_{m} \Theta_{a m}^{\sharp n}$.

\subsection{A two-parameter family of connections $\Theta^{(\epsilon, \rho)}$ on $\mathcal{X}$}

As reviewed in appendix A, the supersymmetry Killing spinor of heterotic supergravity is covariantly constant with respect to the connection

$$
\Theta_{m}^{\mathrm{B}}=\Theta_{m}^{\mathrm{LC}}-\frac{1}{2} H_{m} .
$$

By using this and writing $J$ as a spinor bilinear, it follows that $J$ is covariantly constant with respect to this connection $\nabla^{\mathrm{B}} J=0$, and so $\Theta^{\mathrm{B}}$ is hermitian. Furthermore, the equation $\nabla^{\mathrm{B}} J=0$ can be expressed in terms of forms, and when so written, in a holomorphic frame, we have that $H=\mathrm{i}(\partial-\bar{\partial}) \omega$, as we see from (A.9) and (A.10). This is stated more generally as $H=\mathrm{d}^{c} \omega$. On the other hand the torsion of $\Theta^{\mathrm{B}}$, as defined in (A.1), is exactly $H$, more precisely $T^{m}{ }_{n p}=H_{n p}^{m}$. Thus, we find that $\Theta^{\mathrm{B}}$ has totally antisymmetric torsion equal to $\mathrm{d}^{c} \omega$. This connection is known in the mathematics literature as the Bismut connection. 
While the supersymmetry spinor is covariantly constant with respect to $\Theta^{\mathrm{B}}$, a different connection $\Theta^{\mathrm{H}}$ appears in the heterotic action (A.7). It is non-hermitian and has torsion given by $-H$. Hence,

We call this the Hull connection.

$$
\Theta_{m}^{\mathrm{H}}=\Theta_{m}^{\mathrm{LC}}+\frac{1}{2} H_{m}
$$

The last term in the moduli space metric (1.18) derives from dimensionally reducing the quantity $\operatorname{Tr}\left|R\left(\Theta^{\mathrm{H}}\right)\right|^{2}$, and our task therefore is to compute the covariant derivative of the Hull connection $\mathfrak{D}_{a} \Theta^{\mathrm{H}}$. However, we will first work in more generality and compute the covariant derivative of a two-parameter family of connections introduced in [17], of which $\Theta^{\mathrm{B}}, \Theta^{\mathrm{H}}$ are special cases. This family also includes the 1-parameter family defined by Gauduchon [18]. We will show that only a 1-parameter subfamily are holomorphic on $\mathbb{X}$, and this includes the Hull connection. To define the family, consider a fixed complex manifold $X$, and on $\mathcal{T}_{X}$ we introduce the connection $\Theta^{(\epsilon, \rho)}$, with $\epsilon, \rho \in \mathbb{R}$ and symbols

$$
\begin{aligned}
& \Theta^{(\epsilon, \rho)}{ }_{\mu}^{\nu}{ }_{\sigma}=\Theta^{\mathrm{LC}}{ }_{\mu}{ }^{\nu} \sigma+\frac{(\epsilon-\rho)}{2} H_{\mu}{ }^{\nu}{ }_{\sigma}, \\
& \Theta^{(\epsilon, \rho)} \bar{\mu}^{\nu}{ }_{\sigma}=\Theta^{\mathrm{LC}} \bar{\mu}^{\nu}{ }_{\sigma}+\frac{(\epsilon-\rho)}{2} H_{\bar{\mu}^{\nu}}{ }^{\sigma}, \\
& \Theta^{(\epsilon, \rho)}{ }_{\mu}^{\bar{\nu}} \sigma=0, \\
& \Theta^{(\epsilon, \rho)} \bar{\mu}^{\bar{\nu}}{ }_{\sigma}=\Theta^{\mathrm{LC}} \bar{\mu}^{\bar{\nu}} \sigma+\frac{(\epsilon+\rho)}{2} H_{\bar{\mu}^{\bar{\nu}} \sigma}{ }^{\bar{\nu}},
\end{aligned}
$$

where $\Theta^{\mathrm{LC}}$ is the Levi-Civita connection and $H=\mathrm{d}^{c} \omega$. The Bismut connection is given by $\Theta^{\mathrm{B}}=\Theta^{(-1,0)}$, the Hull connection by $\Theta^{\mathrm{H}}=\Theta^{(1,0)}$ and the Chern connection by $\Theta^{\mathrm{Ch}}=$ $\Theta^{(0,-1)}$. Furthermore, when $\rho+\epsilon=-1$ this reduces to the 1-parameter family of Gauduchon.

To compute $\mathfrak{D}_{a} \Theta^{(\epsilon, \rho)}$ we extend $\Theta$ to $\mathbb{X}$ as follows. Firstly, we consider the Levi-Civita connection $\Theta^{\mathrm{LC}}$ on $\mathbb{X}$. In terms of an arbitrary basis of vectors, denoted $e_{P}$, using (A.2) it has symbols given by

$$
\begin{aligned}
\mathbb{\Theta}_{P}^{\mathrm{LC} Q} R= & \frac{1}{2} \mathfrak{g}^{Q S}\left(e_{P}\left(\mathfrak{g}_{S R}\right)+e_{R}\left(\mathfrak{g}_{S P}\right)-e_{S}\left(\mathfrak{g}_{P R}\right)\right) \\
& -\frac{1}{2} \mathfrak{g}^{Q S}\left(\left[e_{P}, e_{S}\right]^{T} \mathfrak{g}_{T R}+\left[e_{R}, e_{S}\right]^{T} \mathfrak{g}_{T P}\right)+\frac{1}{2}\left[e_{P}, e_{R}\right]^{Q} .
\end{aligned}
$$

For the e-basis $e_{P}=\left(e_{a}, \partial_{m}\right)$ we get

$$
\begin{aligned}
\mathbb{\Theta}^{\mathrm{LC} n}{ }_{k} & =e^{m} \Gamma_{m}^{\mathrm{LC} n}{ }_{k}+\mathrm{d} y^{a}\left(\partial_{k} c_{a}{ }^{n}+\frac{1}{2} g^{n l} \mathfrak{D}_{a} g_{l k}\right), \\
\mathbb{\Theta}^{\mathrm{LC} b}{ }_{k} & =-\frac{1}{2} e^{m} g^{\sharp b d} \mathfrak{D}_{d} g_{m k}-\frac{1}{2} \mathrm{~d} y^{a} g^{\sharp b d} S_{a d}{ }^{l} g_{l k}, \\
\mathbb{\Theta}^{\mathrm{LC} n}{ }_{c} & =\frac{1}{2} e^{m} g^{n l} \mathfrak{D}_{c} g_{l m}+\frac{1}{2} \mathrm{~d} y^{a} S_{a c}{ }^{n}, \\
\mathbb{\Theta}^{\mathrm{LC} b}{ }_{c} & =-\frac{1}{2} e^{m} g^{\sharp b d} S_{c d}{ }^{l} g_{l m}+\mathrm{d} y^{a} \Gamma^{\sharp \mathrm{LC}}{ }_{a c}{ }^{b},
\end{aligned}
$$

where $\Gamma^{\mathrm{LC}}$ and $\Gamma^{\sharp \mathrm{LC}}$ are the standard expressions, see (A.3), in terms of $g_{m n}$ and $g_{a b}^{\sharp}$ respectively; $\mathfrak{D}_{a} g_{m n}=\left(\bigoplus_{a}^{\sharp} g\right)_{m n}=\left(\mathcal{L}_{e_{a}} g\right)_{m n}$ and $(2.20)$ provides us with an expression 
in components

$$
\mathfrak{D}_{a} g_{m n}=e_{a}\left(g_{m n}\right)-c_{a}{ }^{k},{ }_{m} g_{k n}-c_{a}{ }^{k},{ }_{n} g_{m k} \cdot
$$

The connection $\mathbb{\Theta}^{(\epsilon, \rho)}$ extends $(4.4)$ to $\mathcal{X}$ in a natural way. The resulting symbols are written below in holomorphic coordinates. We have used (4.6), $H=\mathbb{d}^{c} \omega$ and the calculation of $\mathbb{d}^{c} \omega$ in $(3.12)$.

- Internal indices purely vertical:

$$
\begin{aligned}
\Theta^{(\epsilon, \rho) \nu_{\sigma}=} & e^{\mu} \Theta_{\mu}^{(\epsilon, \rho) \nu}{ }_{\sigma}+e^{\bar{\mu}} \Theta_{\bar{\mu}}^{(\epsilon, \rho) \nu}{ }_{\sigma}+\mathrm{d} y^{\alpha}\left(\partial_{\sigma} c_{\alpha}{ }^{\nu}+\frac{(1+\epsilon-\rho)}{2} g^{\nu \bar{\lambda}} \mathfrak{D}_{\alpha} g_{\sigma \bar{\lambda}}\right)+ \\
& +\frac{(1-\epsilon+\rho)}{2} \mathrm{~d} y^{\bar{\alpha}} g^{\nu \bar{\lambda}} \mathfrak{D}_{\bar{\alpha}} g_{\sigma \bar{\lambda}}, \\
\Theta^{(\epsilon, \rho) \bar{\nu}_{\sigma}=} & e^{\bar{\mu}} \Theta_{\bar{\mu}}^{(\epsilon, \rho) \bar{\nu}}+(1+\epsilon+\rho) \mathrm{d} y^{\bar{\alpha}} g^{\bar{\nu} \lambda} \Delta_{\bar{\alpha}[\lambda \sigma]} .
\end{aligned}
$$

- Internal indices of mixed type, upper index horizontal:

$$
\begin{aligned}
\mathbb{\Theta}^{(\epsilon, \rho) \beta_{\sigma}=} & -e^{\mu} g^{\sharp \beta \bar{\delta}}\left(\Delta_{\bar{\delta}(\mu \sigma)}+(\epsilon-\rho) \Delta_{\bar{\delta}[\mu \sigma]}\right)-\frac{(1-\epsilon+\rho)}{2} e^{\bar{\mu}} g^{\sharp \beta \bar{\delta}} \mathfrak{D}_{\bar{\delta}} g_{\sigma \bar{\mu}}+ \\
& +\frac{(1+\epsilon-\rho)}{2} \mathrm{~d} y^{\alpha} g^{\sharp \beta \bar{\delta}} S_{\bar{\delta} \alpha}{ }^{\bar{\lambda}} g_{\sigma \bar{\lambda}}+\frac{(1-\epsilon+\rho)}{2} \mathrm{~d} y^{\bar{\alpha}} g^{\sharp \beta \bar{\delta}} S_{\bar{\delta} \bar{\alpha}} \bar{\lambda} g_{\sigma \bar{\lambda}}, \\
\mathbb{\Theta}^{(\epsilon, \rho) \bar{\beta}}= & -\frac{(1+\epsilon+\rho)}{2} e^{\bar{\mu}} g^{\sharp \bar{\beta} \delta} \mathfrak{D}_{\delta} g_{\sigma \bar{\mu}}+\frac{(1+\epsilon+\rho)}{2} \mathrm{~d} y^{\bar{\alpha}} g^{\sharp \bar{\beta} \delta} S_{\delta \bar{\alpha}^{\bar{\lambda}}} g_{\sigma \bar{\lambda}} .
\end{aligned}
$$

- Internal indices of mixed type, upper index vertical:

$$
\begin{aligned}
\mathbb{\Theta}^{(\epsilon, \rho) \nu_{\gamma}=} & \frac{(1-\epsilon+\rho)}{2} e^{\mu} g^{\nu \bar{\lambda}} \mathfrak{D}_{\gamma} g_{\mu \bar{\lambda}}+e^{\bar{\mu}} g^{\nu \bar{\lambda}}\left(\Delta_{\gamma(\bar{\mu} \bar{\lambda})}+(\epsilon-\rho) \Delta_{\gamma[\bar{\mu} \bar{\lambda}]}\right) \\
& +\frac{(1-\epsilon+\rho)}{2} \mathrm{~d} y^{\alpha} S_{\alpha \gamma}{ }^{\nu}+\frac{(1+\epsilon-\rho)}{2} \mathrm{~d} y^{\bar{\alpha}} S_{\bar{\alpha} \gamma}{ }^{\nu}, \\
\mathbb{\Theta}^{(\epsilon, \rho) \bar{\nu}}{ }_{\gamma}= & \frac{(1+\epsilon+\rho)}{2} e^{\bar{\mu}} g^{\bar{\nu} \lambda} \mathfrak{D}_{\gamma} g_{\lambda \bar{\mu}}+\frac{(1+\epsilon+\rho)}{2} \mathrm{~d} y^{\bar{\alpha}} S_{\bar{\alpha} \gamma}{ }^{\bar{\nu}} .
\end{aligned}
$$

- Internal indices purely horizontal:

$$
\begin{aligned}
& \mathbb{\Theta}^{(\epsilon, \rho) \beta_{\gamma}}=\frac{(1-\epsilon+\rho)}{2} e^{\mu} g^{\sharp \beta \bar{\delta}} S_{\bar{\delta} \gamma}{ }^{\bar{\lambda}} g_{\mu \bar{\lambda}}+\frac{(1-\epsilon+\rho)}{2} e^{\bar{\mu}} g^{\sharp \beta \bar{\delta}} S_{\bar{\delta} \gamma}{ }^{\lambda} g_{\lambda \bar{\mu}}+\mathrm{d} y^{\alpha} \Theta_{\alpha}^{\sharp}{ }^{\beta}, \\
& \mathbb{\Theta}^{(\epsilon, \rho) \bar{\beta}_{\gamma}}=\frac{(1+\epsilon+\rho)}{2} e^{\bar{\mu}} g^{\sharp \bar{\beta} \delta} S_{\delta \gamma}{ }^{\lambda} g_{\lambda \bar{\mu}} .
\end{aligned}
$$

Some comments are in order. First, the symbols $\Theta^{(\epsilon, \rho)}$ coincide with the symbols $\Theta^{(\epsilon, \rho)}$ on the fibre $X$ when all three indices are vertical. This is not the case if the symbols are expressed in the coordinate basis $\left\{\partial_{a}, \partial_{m}\right\}$. Second, when all indices are horizontal, $\Theta$ coincides with $\Theta^{\sharp}$ the connection symbols formed from $g^{\sharp}$. As $g^{\sharp}$ is Kähler the connection is the unique hermitian torsionless connection on $M$, whose only nonvanishing components are

$$
\Theta_{\alpha}^{\sharp}{ }_{\alpha}^{\beta}=g^{\sharp \beta \bar{\delta}} \partial_{\alpha} g_{\bar{\delta} \gamma}^{\sharp} \text {. }
$$

Finally, we denote the split $\nabla$ according to tangibility as follows

$$
\nabla=\nabla+\nabla^{\sharp}=e^{m} \nabla_{m}+\mathrm{d} y^{a} \nabla_{a}^{\sharp} .
$$




\subsection{The covariant derivative of $\Theta^{(\epsilon, \rho)}$}

We now compute $\mathfrak{D}_{\alpha} \Theta^{(\epsilon, \rho)}$ to zeroth order in $\alpha^{\prime}$. We will find that only when $\epsilon-\rho=1$ is the connection holomorphic, that is $\mathbb{R}^{(0,2)}=0$. For the remainder of the paper we set $S_{a b}=0$, and work in harmonic gauge, the conventional choice in supergravity: $\nabla^{m} \delta g_{m n}=0$ where $\nabla_{m}=\partial_{m}+\Theta_{m}$ is computed with respect to the affine spin connection on $X$ that is discussed in appendix A. This gauge fixing decomposes into

$$
\Delta_{\alpha}{ }^{\mu} \omega_{\mu}=0, \quad \nabla_{\mu} \Delta_{\alpha}{ }^{\mu}=0, \quad \partial_{m}\left(\omega^{\mu \bar{\nu}} \mathfrak{D}_{\alpha} \omega_{\mu \bar{\nu}}\right)=0,
$$

provided $X$ has $h^{(0,2)}=0$. Interestingly, without vanishing curvature $S=0$ and gauge fixing, the connection is not holomorphic for any choice of $\epsilon, \rho$.

First, we demand that the connection is holomorphic $\mathcal{D}_{\alpha} \Theta_{\mu}=0$. Using (4.3), we find the following components are not immediately zero:

$$
\mathfrak{D}_{\alpha} \Theta^{(\epsilon, \rho)}{ }_{\mu}{ }^{\nu}{ }_{\sigma}=\frac{(1-\epsilon+\rho)}{2 \mathrm{i}} g^{\nu \bar{\lambda}} \nabla_{\mu} \mathfrak{D}_{\alpha} \omega_{\sigma \bar{\lambda}}, \quad \mathfrak{D}_{\alpha} \Theta^{(\epsilon, \rho)}{ }_{\mu}^{\bar{\nu}} \bar{\sigma}=-\frac{(1-\epsilon+\rho)}{2 \mathrm{i}} g^{\bar{\nu} \lambda} \nabla_{\mu} \mathfrak{D}_{\alpha} \omega_{\lambda \bar{\sigma}} .
$$

We see that the covariant derivatives of the variations appear

$$
\nabla_{\sigma} \Delta_{\alpha \bar{\mu}}{ }^{\nu}=\partial_{\sigma} \Delta_{\alpha \bar{\mu}}{ }^{\nu}+\Theta_{\sigma}{ }_{\lambda}{ }_{\lambda} \Delta_{\alpha \bar{\mu}}{ }^{\lambda}, \quad \nabla_{\bar{\mu}} \mathfrak{D}_{\alpha} \omega_{\sigma \bar{\nu}}=\partial_{\bar{\mu}} \mathfrak{D}_{\alpha} \omega_{\sigma \bar{\nu}}-\Theta_{\bar{\mu}}{ }^{\bar{\lambda}} \bar{\nu} \mathfrak{D}_{\alpha} \omega_{\sigma \bar{\lambda}} .
$$

For the connection to be holomorphic we need to set $\epsilon-\rho=1$. It can be checked that this relation is sufficient to ensure that $\mathbb{R}^{(0,2)}=0$. So we have found a 1-parameter family of holomorphic connections on $\mathbf{X}$.

Computing, we find the following non-zero components for the physical deformations $\mathfrak{D}_{\alpha} \Theta_{\bar{\mu}}$ :

$$
\begin{aligned}
& \mathfrak{D}_{\alpha} \Theta^{(\epsilon, \epsilon-1)} \bar{\mu}^{\nu}{ }_{\sigma}=\nabla_{\sigma} \Delta_{\alpha \bar{\mu}}{ }^{\nu}+i \nabla^{\nu} \mathfrak{D}_{\alpha} \omega_{\sigma \bar{\mu}} \\
& \mathfrak{D}_{\alpha} \Theta^{(\epsilon, \epsilon-1)} \bar{\mu}^{\bar{\nu}} \bar{\sigma}=-g^{\bar{\nu} \lambda}\left(\nabla_{\lambda} \Delta_{\alpha \bar{\mu}}{ }^{\rho}+i \nabla^{\rho} \mathfrak{D}_{\alpha} \omega_{\lambda \bar{\mu}}\right) g_{\rho \bar{\sigma}} .
\end{aligned}
$$

Before we continue, let us pause to make some comments. Firstly, we have not computed terms which have vertical indices, such as $\mathfrak{D}_{\alpha} \Theta_{\mu}{ }^{\alpha}$, as they do not appear in (1.18).

Second, it is straightforward to show that $\mathfrak{D}_{\alpha} \Theta$ satisfies the Atiyah condition:

$$
\nabla^{(0,1)} \mathfrak{D}_{\alpha} \Theta^{(0,1)}=\Delta_{\alpha}{ }^{\mu} R_{\mu} .
$$

Third, for the Hull connection $(\epsilon, \rho)=(1,0)$ if we compute the covariant derivative of the fibre metric, we find it vanishes since we have set $S$ to zero:

$$
\nabla_{\alpha}\left(\mathrm{d} s_{X}^{2}\right)=\nabla_{\alpha}\left(2 g_{\mu \bar{\nu}} e^{(\mu} \otimes e^{\bar{\nu})}\right)=-2 g_{\mu \bar{\nu}}\left(S_{\alpha \bar{\beta}^{\mu}}{ }^{\mu} \mathrm{d} y^{\bar{\beta}} \otimes e^{\bar{\nu}}+S_{\alpha \bar{\beta}}{ }^{\bar{\nu}} e^{\mu} \otimes \mathrm{d} y^{\bar{\beta}}\right)=0 .
$$

These covariant derivatives do not mix components of the fibre metric with components of the base metric under parallel transport along the moduli space.

Fourth, the extended connection $\Theta$ defines a covariant derivative of tensors, and it might be tempting to interpret this parallel transport as the appropriate deformation theory of tensors. However, this does not reduce to known expressions derived in [1] for the appropriate deformations of tensors on $X$. Note also, if one were to impose that $\nabla$ and $\pi$ commute, then this would imply $\mathbb{\Theta}^{m}{ }_{a}$ and $\mathbb{\Theta}^{a}{ }_{m}$ vanish. This would mean that $\mathfrak{D}_{\alpha} g_{\mu \bar{\nu}}$ and $\Delta_{\alpha \bar{\mu}}{ }^{\nu}$ both vanish, which is a condition we do not want. 


\subsection{The contribution of $\mathfrak{D}_{\alpha} \Theta$ to the moduli space metric}

We are now in a position to compute the last term of (1.18). The connection in that metric is the Hull connection $(\epsilon, \rho)=(1,0)$, though in fact $\epsilon$ drops out of the following calculation and so it is valid for a 1-parameter family. The integration is evaluated for a fixed point $y \in M$ giving a simplifying rule $e^{m} \rightarrow \mathrm{d} x^{m}$.

We use the result (4.7) to find

$$
\operatorname{Tr}\left(\mathfrak{D}_{\alpha} \Theta \star \mathfrak{D}_{\bar{\beta}} \Theta\right)=2\left(\nabla^{(1,0)} \Delta_{\alpha}{ }^{\mu}+i \nabla^{\mu} \mathfrak{D}_{\alpha} \omega^{(1,1)}\right) \star\left(\nabla^{(0,1)} \Delta_{\bar{\beta}}^{\bar{\nu}}-i \nabla^{\bar{\nu}} \mathfrak{D}_{\bar{\beta}} \omega^{(1,1)}\right) g_{\mu \bar{\nu}},
$$

where $\nabla^{(1,0)} \Delta_{\alpha}{ }^{\mu}=\mathrm{d} x^{\nu} \nabla_{\nu} \Delta_{\alpha}{ }^{\mu}$.

Using $\mathfrak{D}_{\alpha} \omega^{(0,2)}=\mathcal{O}\left(\alpha^{\prime}\right)$ we find

$$
\begin{aligned}
-\frac{\alpha^{\prime}}{4 V} & \int_{X} \operatorname{Tr}\left(\mathfrak{D}_{\alpha} \Theta \star \mathfrak{D}_{\bar{\beta}} \Theta\right)= \\
& -\frac{\alpha^{\prime}}{2 V} \int_{X} \nabla^{(1,0)} \Delta_{\alpha}{ }^{\mu} \star \nabla^{(0,1)} \Delta_{\bar{\beta}}^{\bar{\nu}} g_{\mu \bar{\nu}}-\frac{\alpha^{\prime}}{2 V} \int_{X} \nabla^{\mu} \mathfrak{D}_{\alpha} \omega \star \nabla_{\mu} \mathfrak{D}_{\bar{\beta}} \omega \\
& +\frac{\mathrm{i} \alpha^{\prime}}{2 V} \int_{X} \nabla^{(1,0)} \Delta_{\alpha}{ }^{\mu} \star \nabla_{\mu} \mathfrak{D}_{\bar{\beta}} \omega-\frac{\mathrm{i} \alpha^{\prime}}{2 V} \int_{X} \nabla_{\bar{\nu}} \mathfrak{D}_{\alpha} \omega \star \nabla^{(0,1)} \Delta_{\bar{\beta}}{ }^{\bar{\nu}}+\mathcal{O}\left(\alpha^{\prime 2}\right) .
\end{aligned}
$$

At this point, we notice a series of useful identities. The variation of the complex structure satisfies

$$
\nabla^{\sigma} \nabla_{\sigma} \Delta_{\alpha}{ }^{\mu}=\Delta_{\alpha}{ }^{\sigma \nu} R_{\sigma \bar{\lambda} \nu}{ }^{\mu} \mathrm{d} x^{\bar{\lambda}} \text { and } \nabla_{\mu} \nabla^{(1,0)} \Delta_{\alpha}{ }^{\mu}=0
$$

where we used the vanishing of the pure part of the curvature tensor for $\Theta: R_{\nu \rho \lambda}{ }^{\mu}=0$.

For the Kähler form variation we find

$$
\nabla_{\sigma} \nabla^{\sigma} \mathfrak{D}_{\alpha} \omega=R_{\mu \bar{\nu}}^{\sigma \bar{\tau}} \mathfrak{D}_{\alpha} \omega_{\sigma \bar{\tau}} \mathrm{d} x^{\mu} \mathrm{d} x^{\bar{\nu}} \quad \text { and } \quad g^{\mu \bar{\nu}} \nabla^{\tau} \nabla_{\bar{\nu}} \mathfrak{D}_{\alpha} \omega_{\tau \bar{\sigma}} \mathrm{d} x^{\bar{\sigma}}=0 .
$$

After integrating by parts, using the terms above and metric compatibility of $\nabla$ we find to first order in $\alpha^{\prime}$ :

$$
-\frac{\alpha^{\prime}}{4 V} \int_{X} \operatorname{Tr}\left(\mathfrak{D}_{\alpha} \Theta \star \mathfrak{D}_{\bar{\beta}} \Theta\right)=\frac{\alpha^{\prime}}{2 V} \int_{X}\left(\Delta_{\alpha \overline{\mu \nu}} \Delta_{\bar{\beta} \rho \sigma}+\mathfrak{D}_{\alpha} \omega_{\rho \bar{\mu}} \mathfrak{D}_{\bar{\beta}} \omega_{\sigma \bar{\nu}}\right) R^{\bar{\mu} \rho \bar{\nu} \sigma} .
$$

This expression agrees in form with that derived in [9].

\section{Deriving the moduli space metric from its Kähler potential}

We derive the moduli space metric from the Kähler potential in a concise manner using extended forms on $\mathbf{X}$. As in the previous section, we set $S_{a b}=0$ and within integrals over $X$ we have the rule $e^{m} \rightarrow \mathrm{d} x^{m}, e_{a} \rightarrow \partial_{a}$.

The moduli space metric $g_{\alpha \bar{\beta}}^{\sharp}$ has the associated Kähler form

$$
\omega^{\sharp}=\mathrm{i} g_{\alpha \bar{\beta}}^{\sharp} \mathrm{d} y^{\alpha} \mathrm{d} y^{\bar{\beta}} \text {. }
$$

We show using $\mathbb{X}$ that

$$
\omega^{\sharp}=\mathrm{i} \mathfrak{D} \overline{\mathfrak{D}} \mathcal{K}, \quad \text { where } \quad \mathcal{K}=\mathcal{K}_{1}+\mathcal{K}_{2}=-\log \left(\frac{4}{3} \int \omega^{3}\right)-\log \left(\mathrm{i} \int \Omega \bar{\Omega}\right) .
$$

That is, we are showing that $\mathcal{K}$ is the Kähler potential for the moduli space metric. 
We adopt the convention that when a universal form appears within an integral over $X$, the only surviving part is that which makes the integrand a top form on $X$. Some useful statements illustrating this are

$$
\begin{aligned}
\int_{X} \omega^{2} \mathbb{F} & =\int_{X} \omega^{2} F=0 \\
\frac{1}{2} \int_{X} \omega^{2} \operatorname{Tr} \mathbb{F}^{2} & =\int_{X} \omega^{2} \operatorname{Tr}\left(F \mathbb{F}_{\alpha \bar{\beta}}-\mathbb{F}_{\alpha} \mathbb{F}_{\bar{\beta}}\right) \mathrm{d} y^{\alpha} \mathrm{d} y^{\bar{\beta}}=\int_{X} \omega^{2} \operatorname{Tr}\left(\mathfrak{D}_{\alpha} \mathcal{A} \mathfrak{D}_{\bar{\beta}} \mathcal{A}^{\dagger}\right) \mathrm{d} y^{\alpha} \mathrm{d} y^{\bar{\beta}} \\
\int_{X} \omega^{2} \mathfrak{D} \overline{\mathfrak{D}} \omega & =\int_{X} \omega^{2} \partial \bar{\partial} \omega=\int_{X} \omega^{2} \partial \bar{\partial} \omega .
\end{aligned}
$$

where we use the relations $\omega^{2} F=0, \mathfrak{D}_{\bar{\beta}} \mathcal{A}=0$ and $\bar{\partial}\left(\omega^{2}\right)=\partial\left(\omega^{2}\right)=0$. We will also use, within the integrand,

$$
\mathfrak{D}\left(\frac{\omega^{2}}{2 V}\right)=-\frac{1}{V} \star \mathfrak{D} \omega
$$

Recall that $\mathbb{d} \Omega=-k^{\sharp} \Omega+$ 久 with $\mathcal{K}=\frac{1}{2} \chi_{\alpha \mu \nu \bar{\rho}} \mathrm{d} y^{\alpha} \mathrm{d} x^{\mu} \mathrm{d} x^{\nu} \mathrm{d} x^{\bar{\rho}}$ and $k^{\sharp}=\mathfrak{D} \mathcal{K}_{2}=\mathrm{d} y^{\alpha} \partial_{\alpha} \mathcal{K}_{2}$ and we use $\mathrm{d} \Omega=\overline{\mathfrak{D}} \Omega=0$.

Consider first the derivatives of $\mathcal{K}_{1}$,

$$
\begin{aligned}
\mathfrak{D} \overline{\mathfrak{D}} \mathcal{K}_{1} & =-\mathfrak{D}\left(\frac{1}{2 V} \int_{X} \omega^{2} \overline{\mathfrak{D}} \omega\right) \\
& =\frac{1}{V} \int_{X} \mathfrak{D} \omega \star \overline{\mathfrak{D}} \omega-\frac{\mathrm{i}}{2 V} \int_{X} \omega^{2} \partial \overline{\mathfrak{D}} \omega \\
& =\frac{1}{V} \int_{X} \mathfrak{D} \omega \star \overline{\mathfrak{D}} \omega-\frac{\alpha^{\prime}}{16 V} \int_{X} \omega^{2}\left(\operatorname{Tr} \mathbb{F}^{2}-\operatorname{Tr} \mathbb{R}^{2}\right) \\
& =\left(\frac{1}{V} \int_{X} \mathfrak{D}_{\alpha} \omega \star \mathfrak{D}_{\bar{\beta}} \omega+\frac{\mathrm{i} \alpha^{\prime}}{8 V} \int_{X} \omega^{2} \operatorname{Tr}\left(\mathfrak{D}_{\alpha} \mathcal{A} \mathfrak{D}_{\bar{\beta}} \mathcal{A}^{\dagger}-\mathfrak{D}_{\alpha} \theta \mathfrak{D}_{\bar{\beta}} \theta^{\dagger}\right)\right) \mathrm{d} y^{\alpha} \mathrm{d} y^{\bar{\beta}},
\end{aligned}
$$

where we have used (5.1) and (5.2).

While for the derivatives of $\mathcal{K}_{2}$ we have

$$
\begin{aligned}
\mathrm{i} \mathfrak{D} \overline{\mathfrak{D}} \mathcal{K}_{2} & =-\mathrm{i} \mathfrak{D}\left(\frac{\int_{X} \Omega \overline{\mathfrak{D} \Omega}}{\int_{X} \Omega \bar{\Omega}}\right) \\
& =\mathrm{i}\left(\frac{\int_{X} \mathfrak{D} \Omega \bar{\Omega} \int_{X} \Omega \overline{\mathfrak{D}} \bar{\Omega}}{\left(\int_{X} \Omega \bar{\Omega}\right)^{2}}-\frac{\int_{X} \mathfrak{D} \Omega \overline{\mathfrak{D}} \bar{\Omega}}{\int_{X} \Omega \bar{\Omega}}\right) \\
& =-\mathrm{i} \frac{\int_{X} \chi_{\alpha} \bar{\chi}_{\bar{\beta}}}{\int_{X} \Omega \bar{\Omega}} \mathrm{d} y^{\alpha} \mathrm{d} y^{\bar{\beta}}
\end{aligned}
$$

where we use $\bar{\partial} \Omega=\bar{\partial} \Omega+\overline{\mathfrak{D}} \Omega=0$ and, in the second line, several terms vanish owing to considerations of holomorphic type.

Finally, combining (5.3) and (5.4), we obtain the desired result

$$
\mathrm{i} \mathfrak{D} \overline{\mathfrak{D}} K=\omega^{\sharp} .
$$




\section{Acknowledgments}

We gratefully acknowledge interesting conversations with Anthony Ashmore, Marc-Antoine Fiset, Nigel Hitchin, Richard Thomas, Eirik Svanes and Dan Waldram. PC, JM and XD wish to acknowledge also the hospitality IISc Bangalore and the String theory, the Mainz Institute for Theoretical Physics where part of this work was done. PC and XD also wish to thank KIAS for hospitality while this work was completed and have been supported by EPSRC grant BKRWDM00. JM is supported by STFC grant ST/L000490/1.

\section{A Heterotic geometry}

The purpose of this section is to establish the notation that we use in this paper, and to review some material derived in [1] and [19].

\section{A.1 Some differential geometry}

This material is standard, and used in section 4. Our notation follows that of [19, 20], and more detail may be found in those references.

Consider a manifold $X$ with metric given by

$$
\mathrm{d} s^{2}=g_{m n} \mathrm{~d} x^{m} \otimes \mathrm{d} x^{n}=2 g_{\mu \bar{\nu}} \mathrm{d} x^{\mu} \otimes \mathrm{d} x^{\bar{\nu}} .
$$

We introduce a basis of orthonormal 1-forms $s^{A}=s^{A}{ }_{m} \mathrm{~d} x^{m}$ so that $\mathrm{d} s^{2}=\delta_{A B} s^{A} \otimes s^{B}$ and an affine spin connection 1-form $\Theta$ which satisfies

$$
\mathrm{d} s^{A}+\Theta_{B}^{A} \wedge s^{B}=\frac{1}{2} T^{A}{ }_{B C} s^{B} \wedge s^{C}=\frac{1}{2} T^{A}{ }_{m n} \mathrm{~d} x^{m} \mathrm{~d} x^{n}, \quad \Theta^{A}{ }_{B}=\Theta_{m}{ }_{B}{ }_{B} \mathrm{~d} x^{m},
$$

where $T_{[B C]}^{A}$ is the torsion. In this section only we use upper case roman letters $A, B, \cdots$ to denote flat Lorentz indices. The ordering of the indices here is important, and without care, can lead to sign errors. In the following, we omit the wedge symbol ' $\wedge$ ' where possible.

The connection $\Theta$ being metric compatible means that $\Theta_{A B}=-\Theta_{B A}$, where the indices are raised and lowered with the flat metric $\delta_{A B}$. The Levi-Civita connection $\Theta^{\mathrm{LC}}$ is the unique connection that is both metric compatible and has vanishing torsion. The Levi-Civita connection has symbols, in a coordinate basis, given by the following transformation law

$$
\Theta^{\mathrm{LC}}{ }_{m}^{A}{ }_{B}=s^{A}{ }_{n} \partial_{m} S_{B}{ }^{n}+s^{A}{ }_{q} \Gamma^{\mathrm{LC}}{ }_{p}^{q}{ }_{m} S_{B}{ }^{p},
$$

where $S_{A}=S_{A}^{p} \partial_{p}$ is a basis of vectors dual to the 1 -forms $s^{A}$. In the coordinate basis $\Gamma^{\mathrm{LC}}$ is symmetric in its lower indices; in other bases this is not necessarily the case. In the literature, the $\Gamma$ symbol is often written with a change of ordering of the indices $\Gamma_{m B}{ }^{A}=\Theta_{m}{ }^{A}{ }_{B}$. Care must be taken with signs and for this reason we largely avoid writing the $\Gamma$ symbols. In the coordinate basis, the symbols of Levi-Civita connection are the Christoffel symbols

$$
\Gamma_{m}^{\mathrm{LC}}{ }_{m}^{p}=\frac{1}{2} g^{p l}\left(\partial_{m} g_{l q}+\partial_{q} g_{l m}-\partial_{l} g_{m q}\right) .
$$


The curvature 2 -form is defined as

$$
R_{B}^{A}=\mathrm{d} \Theta^{A}{ }_{B}+\Theta^{A}{ }_{C} \Theta^{C}{ }_{B}=\frac{1}{2} R_{B C D}^{A} s^{C} s^{D}=\frac{1}{2} R_{B m n}^{A} \mathrm{~d} x^{m} \mathrm{~d} x^{n} .
$$

We will have a need also for spinors. We denote the hermitian gamma matrices on $X$ by $\gamma^{m}$; these satisfy the Clifford algebra $\left\{\gamma^{m}, \gamma^{n}\right\}=2 g^{m n}$. The anti-symmetrised product of $k$ gamma matrices is

$$
\gamma^{m_{1} \cdots m_{k}}=\gamma^{\left[m_{1}\right.} \cdots \gamma^{\left.m_{k}\right]},
$$

and the covariant derivative acting on a spinor $\varepsilon$ on $X$ is

$$
\nabla_{m} \varepsilon=\partial_{m} \varepsilon+\frac{1}{4} \Theta_{m A B} \gamma^{A B} \varepsilon
$$

Suppose $\varepsilon$ is a non-vanishing Weyl spinor on $X$ and is covariantly constant $\nabla_{m} \varepsilon=0$. We normalise so that $\varepsilon^{\dagger} \varepsilon=1$. We can then use $\varepsilon$ to define tensors as spinor bilinears. The complex structure $J=J_{m}^{n} \mathrm{~d} x^{m} \otimes \partial_{n}=J_{A}^{B} s^{A} \otimes S_{B}$ will also be relevant. As a spinor bilinear it is

$$
J_{m}^{n}=-i \varepsilon^{\dagger} \gamma_{m}^{n} \varepsilon
$$

It is covariantly constant

$$
\nabla_{m} J=\left(\partial_{m} J_{A}^{B}+\Theta_{m}{ }^{A} C J_{B}{ }^{C}-\Theta_{m}{ }^{C}{ }_{B} J_{C}{ }^{A}\right) s^{A} \otimes S_{B}=0 .
$$

It can be shown that the Nijenhuis tensor of $J$ vanishes [21], so the manifold is complex. We denote its complex coordinates $x^{\mu}, x^{\bar{\nu}}$, then $J_{\mu}{ }^{\nu}=\mathrm{i} \delta_{\mu}{ }^{\nu}$ and $J_{\bar{\mu}}{ }^{\bar{\nu}}=-\mathrm{i} \delta_{\bar{\mu}}{ }^{\bar{\nu}}$. The compatibility equation between the hermitian form $\omega_{m n}$, metric $g_{m n}$ and complex structure $J_{m}{ }^{n}$ is

$$
\omega_{m n}=J_{m}^{p} g_{p n}
$$

In complex coordinates $\omega_{\mu \bar{\nu}}=\mathrm{i} g_{\mu \bar{\nu}}$.

\section{A.2 Heterotic action and supersymmetry variations}

The supergravity action, correct to up to and including $\alpha^{\prime 2}$ is the following [22, 23]:

$$
S=\frac{1}{2 \kappa_{10}^{2}} \int \mathrm{d}^{10} X \sqrt{g_{10}} e^{-2 \Phi}\left\{\mathcal{R}-\frac{1}{2}|H|^{2}+4(\partial \Phi)^{2}-\frac{\alpha^{\prime}}{4}\left(\operatorname{Tr}|F|^{2}-\operatorname{Tr}\left|R\left(\Theta^{\mathrm{H}}\right)\right|^{2}\right)\right\}+\mathcal{O}\left(\alpha^{\prime 3}\right) .
$$

The 10D Newton constant is denoted by $\kappa_{10}, g_{10}=-\operatorname{det}\left(g_{M N}\right), \Phi$ is the $10 \mathrm{D}$ dilaton and $\mathcal{R}$ is the Ricci scalar evaluated using the Levi-Civita connection.

The point-wise inner product on $p$-forms is

$$
|T|^{2}=\frac{1}{p !} g^{M_{1} N_{1}} \ldots g^{M_{p} N_{p}} T_{M_{1} \ldots M_{p}} T_{N_{1} \ldots N_{p}} .
$$

Thus the curvature squared terms correspond to

$$
\operatorname{Tr}|F|^{2}=\frac{1}{2} \operatorname{Tr} F_{M N} F^{M N} \quad \text { and } \quad \operatorname{Tr}\left|R\left(\Theta^{\mathrm{H}}\right)\right|^{2}=\frac{1}{2} \operatorname{Tr} R_{M N P Q}\left(\Theta^{\mathrm{H}}\right) R^{M N P Q}\left(\Theta^{\mathrm{H}}\right),
$$


where the Riemann curvature is evaluated using

$$
\Theta^{\mathrm{H}}=\Theta^{\mathrm{LC}}+\frac{1}{2} H
$$

Using (A.1) this connection has torsion given by

$$
T^{A}{ }_{M N}=-H^{A}{ }_{M N} \text {. }
$$

The connection $\nabla^{\mathrm{H}}$ has torsion with the opposite sign to the connection $\nabla^{\mathrm{B}}$ which appears in the supersymmetry variations. In string frame, these are

$$
\begin{aligned}
\delta \Psi_{M} & =\nabla^{\mathrm{B}} \varepsilon=\nabla_{M}^{\mathrm{LC}} \varepsilon-\frac{1}{4} H_{M} \varepsilon=0, \\
\delta \lambda & =-\frac{1}{2}(\not \partial \Phi) \varepsilon+\frac{1}{4} H \varepsilon=0, \\
\delta \chi & =-\frac{1}{2} \not F \varepsilon=0,
\end{aligned}
$$

corresponding to the gravitino, dilatino and gaugino variations. We have introduced

$$
H_{M}=\frac{1}{2} H_{M N P} \Gamma^{N P}, \quad H=\frac{1}{3 !} H_{M N P} \Gamma^{M N P}, \quad \not H=\frac{1}{2} F_{M N} \Gamma^{M N}, \quad \not \partial=\Gamma^{M} \partial_{M} .
$$

We will always assume the dilaton is constant, and work in weakly coupled perturbative string theory at large radius. The spacetime geometry is $\mathbb{R}^{3,1} \times X$ where the manifold $X$ is compact. The gamma matrices $\Gamma^{M}$ and spinor $\varepsilon$ are decomposed in a manner compatible with this direct product

$$
\Gamma^{e}=\gamma^{e} \otimes 1, \quad \Gamma^{m}=\gamma^{5} \otimes \gamma^{m}, \quad \varepsilon=\zeta_{+} \otimes \eta_{+}+\zeta_{-} \otimes \eta_{-},
$$

where $e, f, \cdots$ are spacetime indices, $\zeta_{ \pm}$are Weyl spinors on $\mathbb{R}^{3,1}$ and $\eta_{ \pm}$are Weyl spinors on $X$ with $\zeta_{+}^{*}=\zeta_{-}$and $\eta_{+}^{*}=\eta_{-}$.

From the calculation in the previous subsection, equation (A.5) requires $J_{m}^{n}$ to be covariantly constant with respect to $\nabla^{\mathrm{B}}$ :

$$
\nabla_{m}^{\mathrm{B}} J_{n}{ }^{p}=\nabla_{m}^{\mathrm{LC}} J_{n}{ }^{p}-\frac{1}{2} H_{m}{ }^{p}{ }_{q} J_{n}{ }^{q}+\frac{1}{2} H_{m}{ }^{q}{ }_{n} J_{q}{ }^{p}=0 .
$$

Contracting with $g_{p r} J_{s}^{r} \mathrm{~d} x^{m} \mathrm{~d} x^{n} \mathrm{~d} x^{s}$, gives

$$
\mathrm{d} \omega+\frac{1}{2} H_{m n p} \mathrm{~d} x^{m} \mathrm{~d} x^{n} J^{p}=0 .
$$

Evaluating this equation in complex coordinates gives

$$
\mathrm{i}(\partial-\bar{\partial}) \omega=H
$$

The manifold $X$ admits a holomorphic volume form $\Omega$ which is related to its hermitian form $\omega$ through a compatibility relation

$$
\frac{1}{3 !} \omega^{3}=\frac{i \Omega \wedge \bar{\Omega}}{\|\Omega\|^{2}} .
$$

The holomorphic form $\Omega$ is $\bar{\partial}$-closed and satisfies $\star \Omega=-i \Omega$, which also means it is $\bar{\partial}$ harmonic. Similarly, $\Omega$ is covariantly constant with respect to $\Theta^{\mathrm{B}}$. This implies that

$$
H_{\mu \nu}{ }^{\nu}=0 \quad \text { and } \quad \partial_{\mu} \log \|\Omega\|^{2}=0 .
$$




\section{A.3 The background field expansion}

The background field expansion is a small fluctuation expansion around a classical background, in which the small fluctuations modulo gauge redundancies are the dynamical quantities of physical interest. We describe this for heterotic theories following the background field method.

Consider a small fluctuation $A \rightarrow A+\delta A$, together with a variation of the gauge transformation $\Phi \rightarrow \Phi(1+\epsilon)$. The total quantity $A+\delta A$ transforms by (1.5). The doctrine of the background field method assigns $A$ the transformation law (1.5) while the fluctuation transforms as

$$
\delta A \rightarrow \Phi\left(\delta A-\mathrm{d}_{A} \epsilon\right) \Phi^{-1} .
$$

This is understood as the composition of two gauge transformations:

- Background gauge transformations

$$
A \rightarrow \Phi A \Phi^{-1}-\mathrm{d} \Phi \Phi^{-1} \text { and } \delta A \rightarrow \Phi \delta A \Phi^{-1} .
$$

- Small gauge transformations

$$
A \rightarrow A \text { and } \delta A \rightarrow \delta A-\mathrm{d}_{A} \epsilon
$$

The former is a classical symmetry of the background, while the latter describes the gauge redundancies of the dynamical variables. Although we have described this using deformations $\delta A$ it equally well applies to other gauge symmetries such as Lorentz or diffeomorphisms. The former acts on all tensors, including $\delta \Theta$ and the metric $\delta g$. The B-field $\delta B$ has an additional symmetry through its Gerbe property.

To first order, variations of heterotic structures are related by differentiating $H=\mathrm{d}^{c} \omega$ and using the cohomology of $X$ :

$$
\begin{aligned}
\mathcal{B}_{\alpha}^{(2,0)} & =\partial \beta_{\alpha}^{1,0}, \\
\mathfrak{D}_{\alpha} \omega^{(2,0)} & =0, \\
\mathcal{B}_{\alpha}^{(0,2)}+\mathrm{i} \mathfrak{D}_{\alpha} \omega^{(0,2)} & =\bar{\partial} \kappa_{\alpha}^{0,1}, \\
\mathcal{B}_{\alpha}^{(1,1)}-\mathrm{i} \mathfrak{D}_{\alpha} \omega^{(1,1)} & =\left(\gamma_{\alpha}+\mathrm{d}\left(\alpha_{\alpha}^{0,1}+\beta_{\alpha}^{1,0}\right)\right)^{(1,1)}, \\
\bar{\partial}\left(\mathcal{B}_{\alpha}^{(1,1)}+\mathrm{i} \mathfrak{D}_{\alpha} \omega^{(1,1)}-\partial \kappa_{\alpha}^{(0,1)}\right) & =2 \mathrm{i} \Delta_{\alpha}{ }^{\mu}\left(\partial_{\mu} \omega-\partial \omega_{\mu}\right)+\frac{\alpha^{\prime}}{2} \operatorname{Tr}\left(\mathfrak{D}_{\alpha} \mathcal{A} F\right)-\frac{\alpha^{\prime}}{2} \operatorname{Tr}\left(\mathfrak{D}_{\alpha} \theta R\right),
\end{aligned}
$$

where $\gamma_{\alpha}^{(1,1)}$ is d-closed $(1,1)$-form. As $\mathcal{B}_{\alpha}$ is defined up to d-closed form, we can absorb the terms involving $\beta_{\alpha}^{(1,0)}$ and $\alpha_{\alpha}^{(0,1)}$. We show in [1] that $\gamma_{\alpha}$ can be absorbed by an $\alpha^{\prime}$-correction to the moduli space coordinates.

Using the covariant derivatives of fields as a basis for a Kaluza-Klein reduction, with the harmonic gauge fixing, gives the moduli space metric (1.18). It is Kähler after taking into account the second order relations between fields. This observation can be generalised to account for the charged matter fields and their fermionic superpartners in order to give the matter field metric as derived in [24]. This normalises physical Yukawa couplings. 


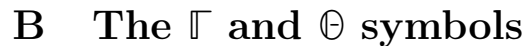

The calculation in this section follows that of [10]. The two relevant bases for $\mathcal{T}_{\mathcal{X}}$ are the coordinate basis and the $e$-basis

$$
\partial_{P}=\left\{\partial_{a}, \partial_{m}\right\}, \quad e_{P}=\left\{e_{a}, e_{m}\right\}=\left\{\partial_{a}-c_{a}{ }^{m} \partial_{m}, \partial_{m}\right\} .
$$

While for $\mathcal{T}_{\not}^{*}$ they are

$$
\mathrm{d} u^{P}=\left\{\mathrm{d} y^{a}, \mathrm{~d} x^{m}\right\}, \quad e^{P}=\left\{e^{a}, e^{m}\right\}=\left\{\mathrm{d} y^{a}, \mathrm{~d} x^{m}+c_{a}^{m} \mathrm{~d} y^{a}\right\} .
$$

We introduce the matrix $e_{Q}{ }^{P}$ and its inverse $E^{P}{ }_{Q}$ as follows

$$
e^{P}=e_{Q}^{P} \mathrm{~d} u^{Q}, \quad e_{Q}=E_{Q}^{P} \partial_{P} .
$$

More explicitly

$$
e_{Q}{ }^{P}=\left(\begin{array}{cc}
\delta_{b}{ }^{a} & 0 \\
c_{b}{ }^{m} & \delta_{n}{ }^{m}
\end{array}\right), \quad E^{P}{ }_{Q}=\left(\begin{array}{cc}
\delta^{a}{ }_{b} & 0 \\
-c_{b}{ }^{m} & \delta^{m}{ }_{n}
\end{array}\right) .
$$

The covariant derivative $\mathbb{\nabla}$ defines symbols $\Theta$ in the $e$-basis and $\mathbb{T}$ in the coordinate basis:

$$
\nabla\left(\partial_{Q}\right)=\mathrm{d} x^{M} \mathbb{T}_{M}{ }^{P}{ }_{Q} \partial_{P} \quad \text { and } \quad \nabla\left(e_{Q}\right)=e^{M} \Theta_{M}{ }^{P}{ }_{Q} e_{P} .
$$

The relation between the symbols follows from

$$
\nabla\left(e_{Q}\right)=e^{M}\left(e_{M}\left(E^{P}{ }_{Q}\right)+E^{N}{ }_{M} E^{S}{ }_{Q} \mathbb{}_{N}{ }^{P}{ }_{S}\right) \partial_{P}=e^{M} \Theta_{M}{ }_{Q}{ }_{Q} E^{P}{ }_{S} \partial_{P},
$$

which we rewrite as

$$
e_{M}\left(E^{P}{ }_{Q}\right)+E^{N}{ }_{M} E^{S}{ }_{Q} \varpi_{N}{ }^{P}{ }_{S}=\mathbb{\Theta}_{M}{ }_{Q}{ }_{Q} E^{P}{ }_{S} .
$$

Under a coordinate transformation, $x \rightarrow \widetilde{x}(x, y)$ and $y \rightarrow \widetilde{y}(y)$, the shift $c_{a}{ }^{m}$ transforms so that the $e$-bases elements rotate in a block diagonal fashion

$$
e_{a} \rightarrow \widetilde{e}_{a}=j_{a}^{b} e_{b}, \quad \partial_{m} \rightarrow \widetilde{\partial}_{m}=j_{m}{ }^{n} \partial_{n}, \quad j_{a}^{b}=\frac{\partial y^{b}}{\partial \widetilde{y}^{a}}, \quad j_{n}{ }^{m}=\frac{\partial x^{n}}{\partial \widetilde{x}^{m}} .
$$

This is viewed as a block diagonal rotation of the $e$-basis

$$
j_{P}{ }^{Q}=\left(\begin{array}{cc}
j_{a}{ }^{b} & 0 \\
0 & j_{m}{ }^{n}
\end{array}\right) .
$$

The $\Theta$ symbols therefore transform as

$$
\widetilde{\Theta}_{M}^{P}{ }_{Q}=\left(\widetilde{e}_{M}\left(j_{Q}{ }^{S}\right)+j_{M}^{T} j_{Q}{ }^{N} \Theta_{T} S_{N}\right) j^{-1 P}{ }_{S} .
$$

The block diagonal structure ensures that symbols such as $\Theta_{m}{ }^{n}$ and $\Theta_{m}{ }^{a}$ transform as tensors, and so their geometric meaning is independent of our choice of $c$ :

$$
\widetilde{\Theta}_{m}{ }^{n}{ }_{a}=j_{m}{ }^{p} j^{-1 n}{ }_{q} j_{a}{ }^{b} \Theta_{p}{ }^{q} b, \quad \widetilde{\Theta}_{m}{ }^{a}{ }_{n}=j_{m}{ }^{p} j_{n}{ }^{q} j^{-1 a}{ }_{b} \Theta_{p}{ }^{b} q .
$$


The geometric interpretation of these symbols is that they are the components of the extrinsic curvature $\chi_{a m}{ }^{n}$ of the fibration of $X$ in $\mathcal{X}$ as described in section 2.7.

The covariant derivative for $c_{a}{ }^{m}$ follows by choosing $M=m, P=p, Q=a$ in (B.2) and using (B.1) together with $\Theta_{m}{ }^{b}{ }_{a}=\mathbb{T}_{m}{ }^{b}{ }_{a}-\mathbb{V}_{m}{ }^{b}{ }_{q} c_{a}{ }^{q}$ :

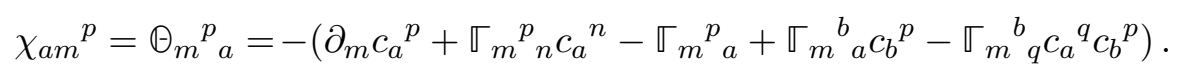

The extrinsic curvature $\chi$ is a tensor and so also is the final expression above. We could, following [10], regard this final expression as the definition of a new covariant derivative of the shift $c_{a}{ }^{m}$.

When $S_{a b}=0$, the expression simplifies

$$
\chi_{a m}{ }^{p}=-\left(\partial_{m} c_{a}^{p}+\mathbb{T}_{m}{ }^{p}{ }_{n} c_{a}^{n}-\mathbb{T}_{m}{ }^{p} a\right) .
$$

We can make some comments on this equation.

1. If $c_{a}{ }^{m}=0$, so that normal vectors are given by $\partial_{a}$, then

$$
\chi_{a m}^{p}=\mathbb{T}_{m}^{p}{ }^{p} .
$$

The following symbols are trivially identical $\Theta_{m}{ }^{p}{ }_{a}=\mathbb{T}_{m}{ }^{p} a$.

2. In complex coordinates if we choose the Levi-Civita connection for $\mathbb{\nabla}$ and use the $\mathbb{T}$ symbols of (B.5), we find

$$
\chi_{\alpha \overline{\mu \rho}}=\Delta_{\alpha(\overline{\mu \rho})} .
$$

3. An analogous calculation gives

$$
\chi_{\alpha \bar{\mu} \rho}=\frac{1}{2} \mathfrak{D}_{\alpha} g_{\rho \bar{\mu}} .
$$

\section{B.1 The $\mathbb{T}$ symbols for the Levi-Civita connection}

These are the $\llbracket$ symbols for the Levi-Civita connection. We first invert the relation (B.2) and decompose the indices, giving

$$
\begin{aligned}
\mathbb{T}^{m}{ }_{n} & =-c_{a}{ }^{m} \mathbb{\Theta}^{b}{ }_{n}+\mathbb{\Theta}^{m}{ }_{n}, \\
\mathbb{T}^{a}{ }_{n} & =\mathbb{\Theta}^{a}{ }_{n}, \\
\mathbb{T}^{m}{ }_{b} & =-c_{a}{ }^{m} \mathbb{\Theta}^{a}{ }_{b}-c_{a}{ }^{m} \Theta^{a}{ }_{n} c_{b}{ }^{n}+\mathbb{\Theta}^{m}{ }_{b}+\mathbb{\Theta}^{m}{ }_{n} c_{b}{ }^{n}+\mathbb{d} c_{b}{ }^{m}, \\
\mathbb{\square}^{a}{ }_{b} & =\mathbb{\Theta}^{a}{ }_{b}+\mathbb{\Theta}^{a}{ }_{n} c_{b}{ }^{n} .
\end{aligned}
$$


Using the symbols for $\mathbb{O}^{\mathrm{LC}}$ from (4.6), we have

$$
\begin{aligned}
& \mathbb{T}_{k}^{n}=\mathrm{d} x^{m}\left(\Gamma_{m}^{\mathrm{LC}}{ }^{n}{ }_{k}+\frac{1}{2} c_{b}{ }^{n} g^{\sharp b d} \mathfrak{D}_{d} g_{m k}\right) \\
& +\mathrm{d} y^{a}\left(c_{a}{ }^{m} \Gamma^{\mathrm{LC}}{ }_{m}{ }^{n}{ }_{k}+c_{a}{ }^{m} c_{b}{ }^{n} g^{\sharp b d} \mathfrak{D}_{d} g_{m k}+\partial_{k} c_{a}{ }^{n}+\frac{1}{2} g^{n l} \mathfrak{D}_{a} g_{l k}+c_{b}{ }^{n} g^{\sharp b d} S_{a d}{ }^{l} g_{l k}\right), \\
& \mathbb{\square}^{b}{ }_{k}=-\frac{1}{2} \mathrm{~d} x^{m} g^{\sharp b d} \mathfrak{D}_{d} g_{m k}-\frac{1}{2} \mathrm{~d} y^{a}\left(c_{a}{ }^{m} g^{\sharp b d} \mathfrak{D}_{d} g_{m k}+g^{\sharp b d} S_{a d}^{l} g_{l k}\right), \\
& \mathbb{\square}^{n}{ }_{c}=\mathrm{d} x^{m}\left(\Gamma_{m}^{\mathrm{LC}}{ }_{k}{ }_{k} c_{c}{ }^{k}+\partial_{m} c_{c}{ }^{k}+\frac{1}{2} c_{b}{ }^{n} g^{\sharp b d} \mathfrak{D}_{d} g_{m k} c_{c}{ }^{k}+\frac{1}{2} g^{n l} \mathfrak{D}_{c} g_{l m}+\frac{1}{2} c_{b}{ }^{n} g^{\sharp b d} S_{c d}{ }^{l} g_{l m}\right) \\
& +\mathrm{d} y^{a}\left(\partial_{a} c_{c}{ }^{n}+\left(\partial_{k} c_{a}{ }^{n}\right) c_{c}{ }^{k}+c_{a}{ }^{m} c_{b}{ }^{n} c_{c}{ }^{k} \Gamma_{m}^{\mathrm{LC}}{ }^{n}{ }_{k}-c_{b}{ }^{n} \Gamma_{a}^{\sharp \mathrm{LC}}{ }_{a}^{b}\right. \\
& +\frac{1}{2} c_{a}{ }^{m} c_{b}{ }^{n} g^{\sharp b d} \mathfrak{D}_{d} g_{m k} c_{c}{ }^{k}+\frac{1}{2} c_{a}{ }^{m} g^{n l} \mathfrak{D}_{c} g_{l m}+\frac{1}{2} c_{c}{ }^{k} g^{n l} \mathfrak{D}_{a} g_{l k} \\
& \left.+\frac{1}{2} S_{a c}{ }^{n}+\frac{1}{2} S_{c d}{ }^{l} c_{a}^{m} c_{b}^{n} g^{\sharp b d} g_{l m}+\frac{1}{2} S_{a d}{ }^{l} c_{b}^{n} c_{c}{ }^{k} g^{\sharp b d} g_{l k}\right), \\
& \mathbb{T}^{b}{ }_{c}=-\frac{1}{2} \mathrm{~d} x^{m}\left(c_{c}{ }^{k} g^{\sharp b d} \mathfrak{D}_{d} g_{m k}-S_{d c}^{l} g^{\sharp b d} g_{l m}\right) \\
& +\mathrm{d} y^{a}\left(\Gamma_{a{ }_{c}}^{\sharp \mathrm{LC}}{ }^{b}-\frac{1}{2} c_{a}{ }^{m} c_{c}{ }^{k} g^{\sharp b d} \mathfrak{D}_{d} g_{m k}+\frac{1}{2} S_{d c}{ }^{l} c_{a}{ }^{m} g^{\sharp b d} g_{l m}-\frac{1}{2} S_{a d} g^{\sharp} g^{\sharp b d} g_{l k} c_{c}{ }^{k}\right) .
\end{aligned}
$$

\section{The Nijenhuis tensor for $\mathbb{X}$}

The Nijenhuis tensor for $\mathbb{l}$ is

$$
N_{\rrbracket}=\left(\rrbracket^{P} \partial_{P} \rrbracket^{Q}-\rrbracket_{P}^{Q} \mathbb{d}^{P}\right) \partial_{Q},
$$

where $u^{P}=\left(y^{a}, x^{m}\right)$ denotes a point in $\mathbb{X}$ and we write $\mathbb{J}^{P}=\sqrt{ }_{S}{ }^{P} \mathrm{~d} u^{S}$. The complex structure is triangular in the coordinate basis:

$\mathbb{J}=J_{m}{ }^{n} e^{m} \otimes e_{n}+J^{\sharp}{ }_{a}^{b} e^{a} \otimes e_{b}=J_{m}{ }^{n} \mathrm{~d} x^{m} \otimes \partial_{n}+\left(c_{a}{ }^{m} J_{m}{ }^{n}-J^{\sharp}{ }_{a}{ }^{b} c_{b}{ }^{n}\right) \mathrm{d} y^{a} \otimes \partial_{n}+J^{\sharp}{ }_{a}^{b} \mathrm{~d} y^{a} \otimes \partial_{b}$.

Thus,

$$
\mathbb{J}_{m}{ }^{a}=0, \quad \mathbb{J}_{a}{ }^{m}=J_{n}{ }^{m} c_{a}{ }^{m}-J_{a}^{\sharp}{ }^{b} c_{b}{ }^{m} .
$$

The terms in (C.1) decompose according to tangibility. In the following, we suppress the $\otimes$ in writing out the tensor structure of $N_{\S}$ to simplify notation, so for example $N_{\S}=$ $\frac{1}{2} N_{\emptyset} P Q^{R} \mathrm{~d} u^{P} \mathrm{~d} u^{Q} \partial_{R}$.

1. The first term, proportional to $\mathrm{d} x^{m} \mathrm{~d} x^{n}$, reduces to that on $X$

$$
\frac{1}{2} N_{\rrbracket m n}{ }^{Q} \mathrm{~d} x^{m} \mathrm{~d} x^{n} \partial_{Q}=N_{J} .
$$

2. The next term has mixed tangibility $\mathrm{d} y^{a} \mathrm{~d} x^{m}$

$$
\begin{aligned}
N_{\rrbracket a m}{ }^{Q} \mathrm{~d} y^{a} \mathrm{~d} x^{m} \partial_{Q}= & N_{J m n}{ }^{q} c_{a}{ }^{m} \mathrm{~d} y^{a} \mathrm{~d} x^{n} \partial_{q}+\left(J_{a}^{\sharp}{ }_{a}^{b} \delta_{p}^{q}-\delta_{a}{ }^{b} J_{p}{ }^{q}\right) e_{b}\left(J_{m}{ }^{p}\right) \partial_{q} \\
& +\left(J^{\sharp}{ }_{a}^{b} J_{p}^{q} \delta_{m}{ }^{n}-J^{\sharp} b^{a} J_{m}{ }^{n} \delta_{p}{ }^{q}\right)\left[e_{n}, e_{b}\right]^{p} \mathrm{~d} y^{a} \mathrm{~d} x^{m} \partial_{q} .
\end{aligned}
$$


where

$$
e_{b}\left(J_{m}^{p}\right)=\partial_{b} J_{m}^{p}-c_{b}^{n} \partial_{n} J_{m}^{p} \quad \text { and } \quad\left[e_{n}, e_{b}\right]=-\left(\partial_{n} c_{b}^{q}\right) \partial_{q} .
$$

We use the projectors of (2.30) to rewrite the $N_{\searrow \text { a }}{ }^{Q}$ components

$$
\begin{aligned}
N_{\Downarrow a m}{ }^{Q} \mathrm{~d} y^{a} \mathrm{~d} x^{m} \partial_{Q}= & N_{J m n}{ }^{q} c_{a}{ }^{m} \mathrm{~d} y^{a} \mathrm{~d} x^{n} \partial_{q}+2 \mathrm{i}\left(P_{a}{ }^{c} Q_{p}{ }^{q}-Q_{a}{ }^{c} P_{p}^{q}\right) e_{c}\left(J_{m}{ }^{p}\right) \partial_{q} \\
& +4\left(P_{a}{ }^{c} P_{m}{ }^{n} Q_{p}{ }^{q}+Q_{a}{ }^{c} Q_{m}{ }^{n} P_{p}{ }^{q}\right)\left[e_{n}, e_{c}\right]^{p} \mathrm{~d} y^{a} \mathrm{~d} x^{m} \partial_{q} .
\end{aligned}
$$

3. The final term of (C.1) has tangibility $\mathrm{d} y^{a} \mathrm{~d} y^{b}$

$$
\begin{aligned}
& \frac{1}{2} N_{\rrbracket a b}{ }^{Q} \mathrm{~d} y^{a} \mathrm{~d} y^{b} \partial_{Q}=\frac{1}{2} N_{J^{\sharp} a b}{ }^{d} \mathrm{~d} y^{a} \mathrm{~d} y^{b} e_{d}+\frac{1}{2} N_{J m n}{ }^{q} c_{a}{ }^{m} c_{b}{ }^{n} \mathrm{~d} y^{a} \mathrm{~d} y^{b} e_{q} \\
& +\left(J^{\sharp}{ }_{a}^{c} e_{c}\left(J_{p}^{q}\right) c_{b}{ }^{p}-J_{m}^{q} e_{a}\left(J_{p}{ }^{m}\right) c_{b}{ }^{p}\right) \mathrm{d} y^{a} \mathrm{~d} y^{b} \partial_{q} \\
& +\left(\delta_{a}^{c} \delta_{b}{ }^{d} \delta_{p}^{q}+J^{\sharp}{ }_{a}^{c} J_{p}^{q} \delta_{b}{ }^{d}-J^{\sharp}{ }_{a}^{c}{J^{\sharp}}_{b}{ }^{d} \delta_{p}{ }^{q}+J^{\sharp}{ }_{b}^{d} J_{p}{ }^{q} \delta_{a}{ }^{c}\right)\left(\partial_{c} c_{d}{ }^{p}\right) \mathrm{d} y^{a} \mathrm{~d} y^{b} \partial_{q} \\
& +\left(\delta_{a}^{c} \delta_{b}^{d} J_{n}^{m} J_{p}^{q}-\delta_{a}^{c}{J^{\sharp}}_{b}^{d}{J_{n}}^{m} \delta_{p}^{q}-{J^{\sharp}}_{a}^{c} \delta_{b}{ }^{d} \delta_{n}{ }^{m}{J_{p}}^{q}+{J^{\sharp}}_{a}^{c}{J^{\sharp}}_{b}^{d} \delta_{n}{ }^{m} \delta_{p}^{q}\right) \\
& \times c_{c}^{n}\left(\partial_{m} c_{d}^{p}\right) \mathrm{d} y^{a} \mathrm{~d} y^{b} \partial_{q} \text {. }
\end{aligned}
$$

In terms of projectors

$$
\begin{aligned}
& \frac{1}{2} N_{\Downarrow a b}{ }^{Q} \mathrm{~d} y^{a} \mathrm{~d} y^{b} \partial_{Q}=\frac{1}{2} N_{J^{\sharp} a b}{ }^{d} \mathrm{~d} y^{a} \mathrm{~d} y^{b} e_{d}+\frac{1}{2} N_{J m n^{q}} c_{a}{ }^{m} c_{b}{ }^{n} \mathrm{~d} y^{a} \mathrm{~d} y^{b} e_{q} \\
& +2 \mathrm{i}\left(P_{a}{ }^{c} Q_{p}{ }^{q}-Q_{a}{ }^{c} P_{p}^{q}\right) e_{c}\left(J_{m}{ }^{p}\right) c_{b}{ }^{m} \mathrm{~d} y^{a} \mathrm{~d} y^{b} \partial_{q}-2\left(P^{c} P^{d} Q_{p}+Q^{c} Q^{d} P_{p}\right)\left[e_{c}, e_{d}\right]^{p} \\
& -4\left(P^{c} P^{d} Q_{p} P_{n}{ }^{m}+Q^{c} Q^{d} P_{p} Q_{n}{ }^{m}+P^{c} Q^{d} Q_{n}{ }^{m} P_{p}+Q^{c} P^{d} P_{n}{ }^{m} Q_{p}\right) c_{c}{ }^{n}\left[e_{m}, e_{d}\right]^{p} \text {. }
\end{aligned}
$$

Gathering (C.2), (C.3) and (C.4), and simplifying we find

$$
\begin{aligned}
N_{\rrbracket}= & \frac{1}{2} N_{J m n}{ }^{q} e^{m} e^{n} e_{q}+2 \mathrm{i} e_{c}\left(J_{m}{ }^{p}\right)\left(P^{\sharp c} e^{m} Q_{p}-Q^{\sharp c} e^{m} P_{p}\right) \\
& -4\left[e_{c}, e_{n}\right]^{p}\left(P^{\sharp c} P^{n} Q_{p}+Q^{\sharp c} Q^{n} P_{p}\right)-2\left[e_{c}, e_{d}\right]^{p}\left(P^{\sharp c} P^{\sharp d} Q_{p}+Q^{\sharp c} Q^{\sharp d} P_{p}\right)+\frac{1}{2} N_{J^{\sharp} a b} e^{a} e^{b} e_{c} .
\end{aligned}
$$

The second and third terms combine in virtue of the relation

$$
\left[P_{a}^{\sharp}, P_{m}\right]^{p} \mathrm{~d} y^{a} e^{m}=-\frac{\mathrm{i}}{2} P^{\sharp c} e_{c}\left(J_{m}{ }^{p}\right) e^{m}+P^{\sharp c} P^{m}\left[e_{c}, e_{m}\right]^{p} .
$$

Note also that

$$
\left[P_{c}^{\sharp}, P_{d}^{\sharp}\right]^{q}=P^{\sharp}{ }_{c}^{a} P^{\sharp}{ }_{d}^{b}\left[e_{a}, e_{b}\right]^{q} .
$$

These relations, together with (C.5), give the final expression

$$
\begin{aligned}
N_{\searrow}= & \frac{1}{2} N_{J m n}{ }^{q} e^{m} e^{n} e_{q}-4\left[P_{a}^{\sharp}, P_{m}\right]^{q} e^{a} e^{m} Q_{q}-4\left[Q_{a}^{\sharp}, Q_{m}\right]^{q} e^{a} e^{m} P_{q} \\
& -2\left[P_{c}^{\sharp}, P_{d}^{\sharp}\right]^{q} e^{c} e^{d} Q_{q}-2\left[Q_{c}^{\sharp}, Q_{d}^{\sharp}\right]^{q} e^{c} e^{d} P_{q}+\frac{1}{2} N_{J^{\sharp} c d} e^{c} e^{d} e_{e} .
\end{aligned}
$$

The first and last term are $N_{J}$ and $N_{J^{\sharp}}$. 


\section{Some examples: deformations of $\omega$ and $\Omega$ within $\mathbb{X}$}

We now illustrate the extensions of some natural tensors in special geometry and calculate their deformations. For this subsection only, $X$ is a Calabi-Yau manifold and we are at the standard embedding. It is a good check that the formalism here reproduces the known deformation theory of a Calabi-Yau manifold.

\section{D.1 The hermitian form $\omega$}

The hermitian form $\omega$ on $\mathbb{X}$ is

$$
\omega=\omega_{m n} e^{m} e^{n}+\omega_{a b}^{\sharp} \mathrm{d} y^{a} \mathrm{~d} y^{b},
$$

with its form is determined by the metric (2.22) on $\mathcal{X}$.

The variation of the hermitian form $\omega$ due to a variation $\mathrm{d} y^{\alpha}$ is

$$
\mathfrak{D}_{\alpha} \omega=e_{\alpha}(\omega)-\left(Đ c_{\alpha}{ }^{m}\right) \omega_{m}
$$

and when decomposed into holomorphic type this yields

$$
\begin{aligned}
& \mathfrak{D}_{\alpha} \omega^{(2,0)}=0, \\
& \mathfrak{D}_{\alpha} \omega^{(1,1)}=\mathfrak{D}_{\alpha} \omega_{\mu \bar{\nu}} e^{\mu} e^{\bar{\nu}}, \quad \mathfrak{D}_{\alpha} \omega_{\mu \bar{\nu}}=e_{\alpha}\left(\omega_{\mu \bar{\nu}}\right)-\left(\partial_{\mu} c_{\alpha}{ }^{\tau}\right) \omega_{\tau \bar{\nu}}, \\
& \mathfrak{D}_{\alpha} \omega^{(0,2)}=i \Delta_{\alpha}{ }^{\nu} \omega_{\nu},
\end{aligned}
$$

Under a small diffeomorphism (A.13) $\delta \omega=\delta y^{a} D_{a} \omega$ transforms in the following way

$$
\delta \omega \rightarrow \delta \omega-\mathcal{L}_{\epsilon} \omega=\delta \omega-\epsilon^{m}(\mathrm{~d} \omega)_{m}-\mathrm{d}\left(\epsilon^{m} \omega_{m}\right)=\delta \omega+\mathrm{d} \varepsilon, \quad \varepsilon=-\epsilon^{m} \omega_{m},
$$

where $\epsilon^{m}$ is a vector and $\varepsilon$ a 1 -form. Harmonic gauge is when $\mathrm{d}^{\dagger} \delta \omega=0$. This requires $\bigoplus_{\alpha}^{\sharp} \omega^{(0,2)}=0$ and $\bigoplus_{\alpha}^{\sharp} \omega^{(1,1)}$ to be harmonic.

\section{D.2 The holomorphic form $\Omega$}

We define the holomorphic three form on $X$ to have an extension which is purely vertical ${ }^{6}$

$$
\Omega=\frac{1}{3 !} \Omega_{\mu \nu \rho} e^{\mu} e^{\nu} e^{\rho}=\frac{1}{3 !} f(x, y) \epsilon_{\mu \nu \rho} e^{\mu} e^{\nu} e^{\rho},
$$

where $\epsilon_{\mu \nu \rho}$ is the constant antisymmetric symbol and the function $f$ depends holomorphically on the coordinates. As $\star \Omega=-i \Omega$, it follows $\Omega$ that is d-harmonic. Supersymmetry implies that it is covariantly constant with respect to the Bismut connection $\nabla^{\mathrm{B}} \Omega=0$. Decomposing according to holomorphic type yields two relations

$$
\begin{aligned}
& \nabla_{\mu}^{\mathrm{B}} \Omega=\left(\partial_{\mu} \log \|\Omega\|^{2}-H_{\mu \nu}{ }^{\nu}\right) \Omega=0, \\
& \nabla_{\bar{\mu}}^{\mathrm{B}} \Omega=-g^{\nu \bar{\lambda}}\left(\partial_{\bar{\mu}} g_{\bar{\lambda} \nu}-\partial_{\bar{\lambda}} g_{\bar{\mu} \nu}\right) \Omega=H_{\bar{\mu} \bar{\lambda}} \overline{\bar{\lambda}}^{\bar{\lambda}} \Omega=0,
\end{aligned}
$$

\footnotetext{
${ }^{6}$ Of course there may be a three form for $\mathbb{X}$ with horizontal components. In this work, they do not play a role and so we do not consider them. This is an example of an object which does not have a natural extension to the universal manifold $\mathbf{X}$.
} 
which are solved by

$$
H_{\mu \nu}^{\nu}=0, \quad \partial_{\mu} \log \|\Omega\|^{2}=0 .
$$

The three-form $\Omega$ is a section of a line bundle over the moduli space $M$ with a $\mathbb{C}^{*}$-gauge symmetry

$$
\Omega \rightarrow \lambda(y) \Omega, \quad \lambda \in \mathbb{C}^{*} .
$$

Now consider variations of $\Omega$, which need to be covariant under (D.3). The derivative $\widetilde{\partial}_{\alpha}^{\sharp} \Omega$ is decomposed into holomorphic type on $X$ :

$$
\mathrm{\partial}_{\alpha}^{\sharp} \Omega=\left(\bigoplus_{\alpha}^{\sharp} \Omega\right)^{(3,0)}+\left(\bigoplus_{\alpha}^{\sharp} \Omega\right)^{(2,1)},
$$

where the superscripts refer to holomorphic type with respect to $J$. Using $\left\{\bigoplus^{\sharp} \bigoplus^{\sharp}\right\}=0$, applying $\bigoplus^{\sharp}$ to $Đ \Omega=0$ and decomposing according to

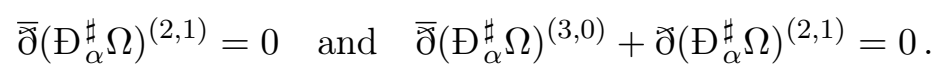

The first equation defines a $\bar{\partial}$-closed form $\chi_{\alpha}=\Delta_{\alpha}{ }^{\mu} \Omega_{\mu}$. For the second equation the Hodge

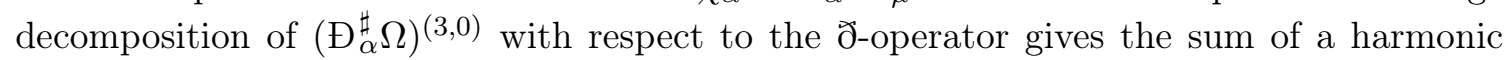
form and a $\partial$-exact term. As $h^{3,0}=1$, the harmonic term is $\Omega$ multiplied by a parameter dependant coefficient $\mathcal{K}_{2 \alpha}$

$$
\left(\bigoplus_{\alpha}^{\sharp} \Omega\right)^{(3,0)}=-\mathcal{K}_{2 \alpha} \Omega+ð \zeta_{\alpha} .
$$

Multiplying this equation by $\bar{\Omega}$ and integrating over $X$, we see the coefficient $\mathcal{K}_{2 \alpha}$ can be written as a derivative

$$
\mathcal{K}_{2 \alpha}=\partial_{\alpha} \mathcal{K}_{2} ; \quad \mathcal{K}_{2}=-\log \left(\int_{X} \mathrm{i} \Omega \bar{\Omega}\right)
$$

Under small diffeomorphisms, there is a transformation law for $\delta \Omega=\delta y^{\alpha} \bigoplus_{\alpha}^{\sharp} \Omega$

$$
\delta \Omega^{(3,0)} \rightarrow \delta \Omega^{(3,0)}-\partial\left(\epsilon^{\mu} \Omega_{\mu}\right) \quad \text { and } \quad \delta \Omega^{(2,1)} \rightarrow \delta \Omega^{(2,1)}-\bar{\partial}\left(\epsilon^{\mu} \Omega_{\mu}\right) .
$$

Comparing this equation with (D.5) we see that we can solve $\epsilon^{\mu} \Omega_{\mu}=\delta y^{\alpha} \zeta_{\alpha}$ with explicit solution given by

$$
\varepsilon^{\nu}=\frac{1}{2\|\Omega\|^{2}} \bar{\Omega}^{\nu \rho \sigma}\left(\delta y^{\alpha} \zeta_{\rho \sigma}+\left(\partial \xi^{(1,0)}\right)_{\rho \sigma}\right)
$$

where $\xi^{(1,0)}$ is an arbitrary one form. With this choice $\delta \Omega^{(3,0)}$ is harmonic. We see that $\xi^{(1,0)}$ is a residual gauge freedom that does not affect $\delta \Omega$.

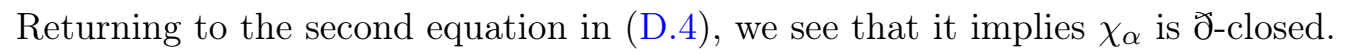

The derivative of $\Omega$ that is covariant both with respect the symmetry (D.3) and diffeomorphisms is

$$
\mathfrak{D}_{\alpha} \Omega=\left(\bigoplus_{\alpha}^{\sharp}+\mathcal{K}_{2 \alpha}\right) \Omega=\chi_{\alpha}=\Delta_{\alpha}{ }^{\mu} \Omega_{\mu} .
$$

Open Access. This article is distributed under the terms of the Creative Commons Attribution License (CC-BY 4.0), which permits any use, distribution and reproduction in any medium, provided the original author(s) and source are credited. 


\section{References}

[1] P. Candelas, X. de la Ossa and J. McOrist, A Metric for Heterotic Moduli, Commun. Math. Phys. 356 (2017) 567 [arXiv: 1605. 05256] [INSPIRE].

[2] M. Atiyah and I. Singer, Dirac operators coupled to vector potentials, Proc. Natl. Acad. Sci. U.S.A. 81 (1984) 2597.

[3] S.K. Donaldson, Polynomial invariants for smooth manifolds, Topology 29 (1990) 257 [INSPIRE].

[4] S.K. Donaldson, The Orientation of Yang-Mills Moduli Spaces and 4-Manifold Topology, J. Diff. Geom. 26 (1987) 397.

[5] E. Witten, Topological Quantum Field Theory, Commun. Math. Phys. 117 (1988) 353 [INSPIRE].

[6] J.A. Harvey and A. Strominger, String theory and the Donaldson polynomial, Commun. Math. Phys. 151 (1993) 221 [hep-th/9108020] [InSPIRE].

[7] J.P. Gauntlett, Low-energy dynamics of $N=2$ supersymmetric monopoles, Nucl. Phys. B 411 (1994) 443 [hep-th/9305068] [INSPIRE].

[8] N. Hitchin, What is a Gerbe?, Not. Am. Math. Soc. 50 (2003) 218.

[9] L. Anguelova, C. Quigley and S. Sethi, The Leading Quantum Corrections to Stringy Kähler Potentials, JHEP 10 (2010) 065 [arXiv: 1007.4793] [INSPIRE].

[10] K. Yano, Differential Geometry on Complex and Almost Complex Spaces, Pergamon Press, (1965).

[11] P. Candelas, Yukawa Couplings Between (2,1) Forms, Nucl. Phys. B 298 (1988) 458 [INSPIRE].

[12] P. Candelas and X. de la Ossa, Moduli Space of Calabi-Yau Manifolds, Nucl. Phys. B 355 (1991) 455 [INSPIRE].

[13] L.B. Anderson, J. Gray and E. Sharpe, Algebroids, Heterotic Moduli Spaces and the Strominger System, JHEP 07 (2014) 037 [arXiv: 1402.1532] [INSPIRE].

[14] X. de la Ossa and E.E. Svanes, Holomorphic Bundles and the Moduli Space of $N=1$ Supersymmetric Heterotic Compactifications, JHEP 10 (2014) 123 [arXiv:1402.1725] [INSPIRE].

[15] X. de la Ossa and E.E. Svanes, Connections, Field Redefinitions and Heterotic Supergravity, JHEP 12 (2014) 008 [arXiv: 1409.3347] [INSPIRE].

[16] M. Garcia-Fernandez, R. Rubio and C. Tipler, Holomorphic string algebroids, arXiv: 1807.10329 [INSPIRE].

[17] A. Otal, L. Ugarte and R. Villacampa, Invariant solutions to the Strominger system and the heterotic equations of motion, Nucl. Phys. B 920 (2017) 442 [arXiv:1604.02851] [InSPIRE].

[18] P. Gauduchon, Hermitian connections and Dirac operators, Bollettino U.M.I 11-B 7 (1997) 257.

[19] T. Eguchi, P.B. Gilkey and A.J. Hanson, Gravitation, Gauge Theories and Differential Geometry, Phys. Rept. 66 (1980) 213 [INSPIRE].

[20] K. Becker, M. Becker and J.H. Schwarz, String theory and M-theory: A modern introduction, Cambridge University Press, (2006). 
[21] A. Strominger, Superstrings with Torsion, Nucl. Phys. B 274 (1986) 253 [INSPIRE].

[22] E.A. Bergshoeff and M. de Roo, The Quartic Effective Action of the Heterotic String and Supersymmetry, Nucl. Phys. B 328 (1989) 439 [INSPIRE].

[23] E. Bergshoeff and M. de Roo, Supersymmetric Chern-Simons Terms in Ten-dimensions, Phys. Lett. B 218 (1989) 210 [inSPIRE].

[24] J. McOrist, On the Effective Field Theory of Heterotic Vacua, Lett. Math. Phys. 108 (2018) 1031 [arXiv: 1606.05221] [INSPIRE]. 